\title{
Experimental investigations of the mechanical properties of wet granular matter
}

\section{Dissertation}

zur Erlangung des mathematisch-naturwissenschaftlichen Doktorgrades

"Doctor rerum naturalium"

der Georg-August-Universität Göttingen

\section{vorgelegt von \\ Mario Scheel}

aus Kempten (Allgäu), Germany

Göttingen, 2009 
D 7

Referent : Prof. Dr. Stephan Herminghaus

Koreferent : Prof. Dr. Marcus Müller

Tag der mündlichen Prüfung : 7. Juli 2009 


\section{Leben ist, was die Welt verändert!}

Mario 



\section{Abstract}

Granular media are omnipresent in every day life. Examples are the cereals we eat, pharmaceutical products, piece goods in industry, or sand on the beach. A dry granular material, like sand, turns into a stiff and moldable material when it is mixed with a wetting liquid. Typically, the stiffness of a wet granulate is extremely robust against variations in the liquid content. To study this behavior we used submillimetric glass beads mixed with a wetting liquid and varied the liquid content between the dry and the completely submerged case.

The mechanical properties of wet granular materials were measured by three different techniques: the critical fluidization acceleration for vertical shaking, the shear stiffness measured in a shear cell, and the tensile strength using a centrifuge. To understand the mechanical properties in terms of the liquid distribution within the granular pile, we investigated the emerging liquid morphologies by optical microscopy and 3D X-ray micro tomography and compared them to corresponding numerical results. Based on these results we have developed a complete picture explaining the liquid morphologies and the resulting mechanical properties of wet granulates. In addition, numerical results regarding the hysteretic transitions between certain liquid morphologies have been confirmed experimentally for different wettabilities and packing densities of the granulate.

The mechanical properties are a result of the particular wetting morphology and are largely insensitive to the grain shape. This was confirmed with granulates consisting of nonspherical, wettable sand grains and spherical, nonwettable basalt beads. 



\section{Kurzzusammenfassung}

Beispiele für Granulate sind überall zu finden: In Nahrungsmitteln, pharmazeutischen Produkten, Stückgut in der Industrie oder Sand am Meeresstrand. Trockener Sand wird durch Zugabe von benetzenden Flüssigkeiten, bespielsweise Wasser, zu einem Material, mit dem Sandburgen gebaut werden können. Die Steifigkeit des Granulats ändert sich kaum, wenn der Flüssigkeitsanteil variiert wird. Um diese Eigenschaften zu untersuchen verwendenten wir submilimeter Glaskugeln, die wir gleichmäßig mit Flüssigkeit vermischten. Der untersuchte Flüssigkeitsbereich reichte vom trockenen zum vollständig gesättigten Granulat.

Die Auswirkungen des Flüssigkeitsanteils auf die mechanischen Eigenschaften des Granulats wurden mit drei unterschiedlichen Messmethoden untersucht. Wir verwendeten die Fluidisierungsschwelle eines vertikal geschüttelten Granulats, die Schersteifigkeit des Granulats gemessen in einer Scherzelle und die Zerreißkraft des Granulats gemessen mit einer Zentrifuge. Um die mechanischen Eigenschaften eines feuchten Granulats zu verstehen, untersuchten wir die Flüssigkeitsverteilung mit optischer Mikroskopie und 3D Röntgenmikrotomographie und verglichen die Strukturen mit Ergebnissen aus numerischen Untersuchungen. Basierend auf diesen Ergebnissen entwickelten wir ein vollständiges Model, welches die auftretende Flüssigkeitsverteilung und die damit verbundenen mechanischen Eigenschaften feuchter Granulate erklärt. Insbesondere wurden numerisch bestimmte Hystereseschleifen, die bei der Umwandlungen zwischen einzelnen Flüssigkeitsstrukturen auftreten, mit experimentellen Beobachtungen verglichen. Zusätzlich wurde der Einfluss der Benetzbarkeit des Granulats durch die beigemischte Flüssigkeit sowie der Packungsdichte experimentell untersucht.

Die mechanischen Eigenschaften der feuchten Granulate sind ein Ergebnis der sich ausbildenden Flüssigkeitsstrukturen. Diese sind stark von der Benetzbarkeit der Körner mit der Flüssigkeit, jedoch nur gering von der Form der Körner abhängig. Diesen Einfluss konnten wir an Granulaten aus benetzbaren Sandkörnern und nicht benetzbaren Basaltkugeln zeigen. 


\section{Contents}

Abstract ............................ i

Kurzzusammenfassung ....................... iii

Contents ................................ vi

Introduction . . . . . . . . . . . . . . . . . . . . . . . 1

1 Physical Background 5

1.1 Sand types and sand phenomena $\ldots \ldots \ldots \ldots \ldots$

1.2 Wetting on a substrate . . . . . . . . . . . . . . . . . 10

1.2 .1 Surface tension, contact angle and Young's law . . . . . . . . . 10

1.2 .2 Capillary force between two beads . . . . . . . . . . . . . . 12

$1.2 .3 \quad$ Wetting from low to large liquid contents $W \ldots \ldots \ldots \ldots$

\begin{tabular}{|lll}
2 & Microscopic measurement techniques & 17
\end{tabular}

$2.1 \quad$ Surface roughness measurement of the grains $\ldots \ldots \ldots \ldots$

2.2 Contact angle and Laplace pressure measurement $\ldots \ldots \ldots \ldots$

2.3 X-ray micro tomography $\ldots \ldots \ldots \ldots \ldots$

3 Mechanical measurements $\quad 33$

3.1 Critical fluidization acceleration measurements . . . . . . . . . . . . 34

$3.1 .1 \quad$ Measurement technique . . . . . . . . . . . . . . . . 34

3.1 .2 Experimental results $\ldots \ldots \ldots \ldots$

3.2 Shear stiffness measurements . . . . . . . . . . . . . . . . . . . . 46

3.2 .1 Measurement technique . . . . . . . . . . . . . 46

3.2 .2 Experimental results $\ldots \ldots \ldots \ldots \ldots$

3.3 Tensile strength measurements $\ldots \ldots \ldots$

$3.3 .1 \quad$ Measurement technique . . . . . . . . . . . . . . . . . 59

3.3 .2 Experimental results $\ldots \ldots \ldots \ldots \ldots$. . . . . . . . . 61 


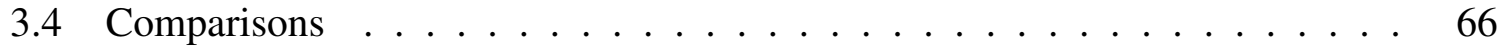

\begin{tabular}{|lrr}
\hline 4 & Liquid distribution in a granular pile & 67
\end{tabular}

$4.1 \quad$ Liquid morphologies in a pile of beads . . . . . . . . . . . . . . . 67

4.2 Number of capillary bridges on a bead . . . . . . . . . . . . . . . 71

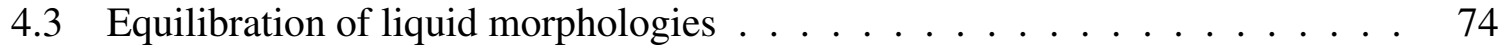

4.4 Laplace pressure in a pile of wet beads . . . . . . . . . . . . . . . . . . 79

4.5 Volume transitions of liquid clusters $\ldots \ldots \ldots \ldots \ldots$

4.5.1 Transition between trimer and capillary bridges in an ideal triangular

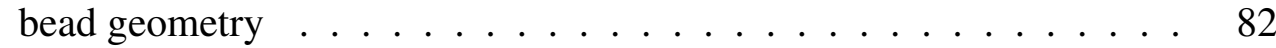

4.5 .2 Transitions between trimers and capillary bridges in a disordered tri-

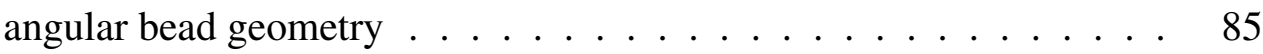

4.5 .3 Transitions of larger clusters $\ldots \ldots \ldots \ldots$

$4.5 .4 \quad$ Forces of single clusters $\ldots \ldots \ldots \ldots$

4.6 Large liquid clusters $\ldots \ldots \ldots 1$

4.7 Characterization of sand in comparison with glass beads _ . . . . . . . . 97

4.8 Open versus compact morphology . . . . . . . . . . . . . . . . . 97

5 Summary and Outlook 101

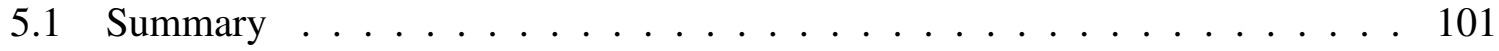

5.2 Outlook . . . . . . . . . . . . . . . . . . . . . . . 102

5.2 .1 Liquid fronts in sandstone $\ldots \ldots \ldots \ldots \ldots$

5.2 .2 Flight path measurements $\ldots \ldots \ldots \ldots$. . . . . . . . 104

5.2 .3 Liquid clusters in a fiber network $\ldots \ldots \ldots \ldots$

$5.2 .4 \quad$ Packing density of cylinders $\ldots \ldots \ldots \ldots$

\begin{tabular}{ll}
\hline Appendices & 108
\end{tabular}

\begin{tabular}{|l|l|}
\hline A Parameter of liquids and particles & 111
\end{tabular}

\begin{tabular}{lr}
\hline Acknowledgments & 121
\end{tabular} 


\section{Introduction}

One of the most important materials in the world are granulates. You will find them at home as cereals on the breakfast table, as wheat flour or popcorn in the kitchen and as burned red clay in the flower pot at the window. Most 'piece goods' in the world from coal to plastic are granulates and they are widely used in industry. In nature you will find granulates like sand in the river, glacier deposits, desert and at the beach on the coastline. Many of us have played on the beach with sand and have build sand castles. It is clear that one's ability to sculpture a sand castle beyond a simple pyramid depends on the increased cohesion of wet sand. The amount of liquid is not important. Only if the tide comes and reaches the castle it will start to collapse. The reason why a wet granulate's mechanical stability is insensitive to the liquid content will be presented in this thesis.

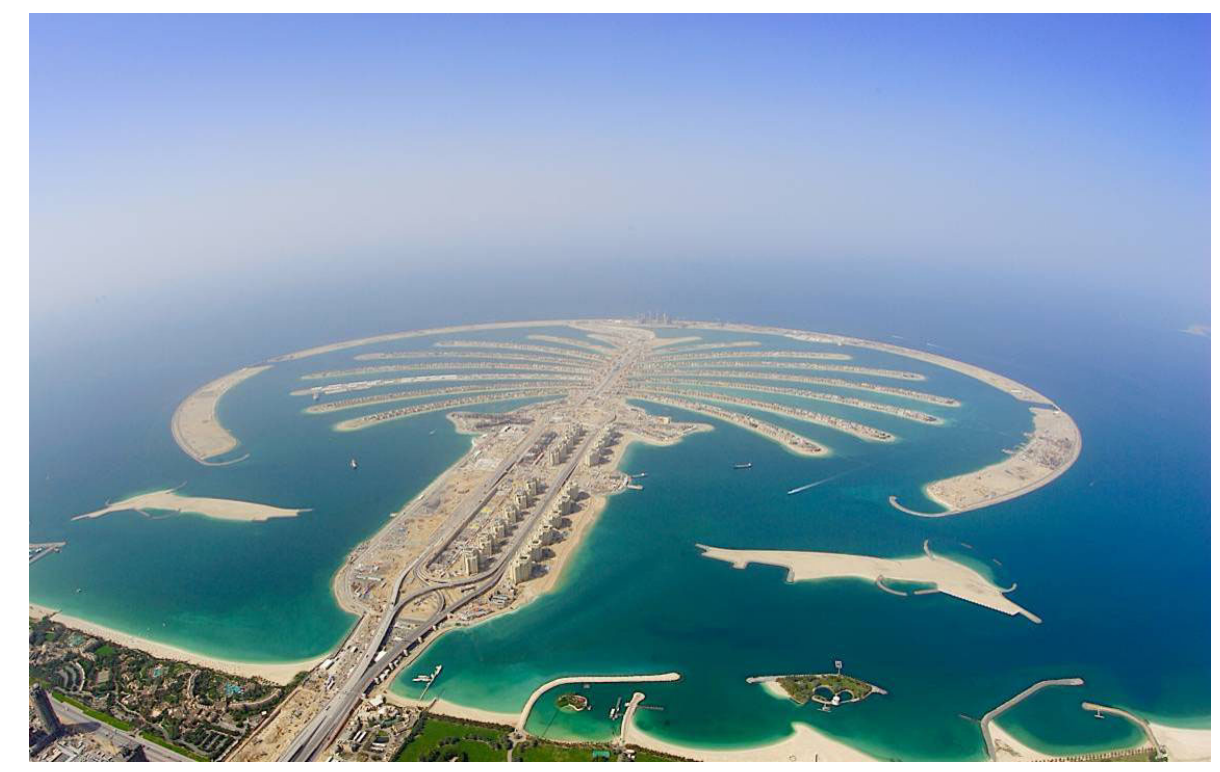

Figure 1: The Palm Jumeirah is constructed in the Persian Gulf in Dubai (copyright Nakheel PJSC). 
In Dubai you can find a huge sand structure on the coast of the Persian Gulf. That 'sand castle' as shown in Figure 1 is called the Palm Jumeirah (copyright Nakheel PJSC). The construction details of the Palm Jumeirah as mentioned from Nakheel PJSC illustrate the unique properties of wet sand. Instead of using normal beach or desert sand with a wide size distribution and small grains, the constructors used sea sand which consist of larger coarse sand. This is important, because the Gulf's waves will cause less erosion compared with normal sand. The sea sand was pumped from the ground with water into huge ships and transported to the 'Palm' where it was pumped out again to form the new island of sand [1]. The sand is sufficiently stable for a man to walk on top shortly after drainage, but for a building this is not stable enough. The packing density of the sand, however, had to be increased by using machines. A huge metal needle, was pressed into the ground and by vibrating the needle and simultaneously pumping a water-sand mixture into the loose sand, you could increase the packing density close to that of a randomly close packed collection of spheres. After this preparation the sand was stable enough to carry large buildings. The same compact sand can be formed by nature without human manipulation within many years.
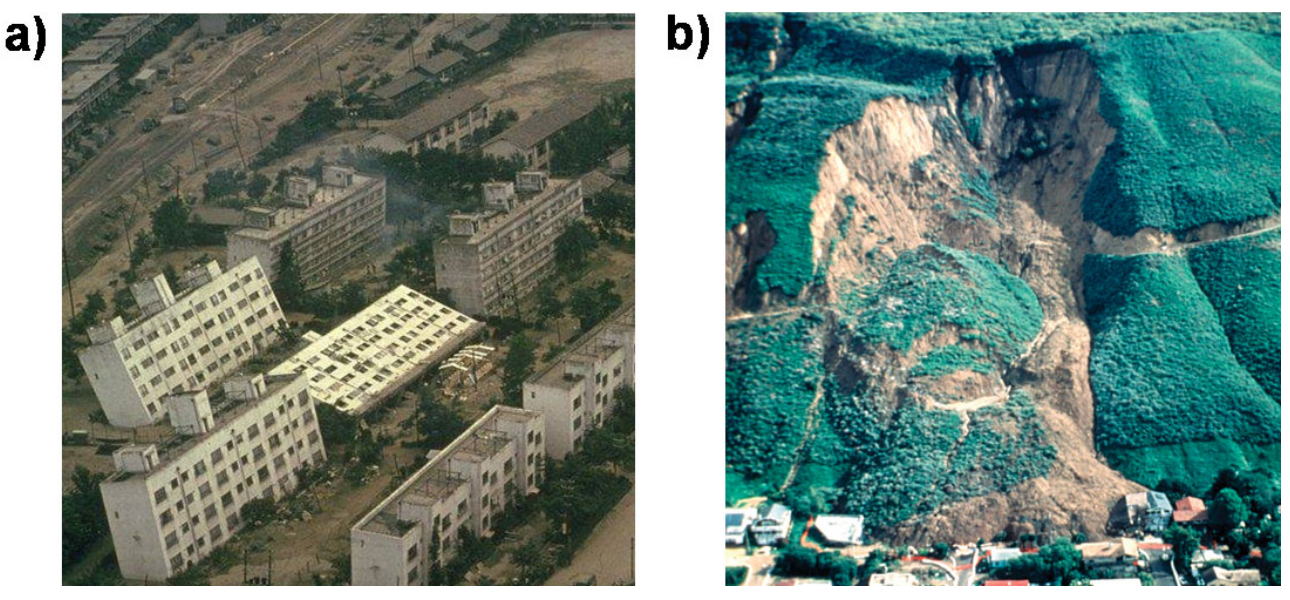

Figure 2: a) Earthquake in Niigata 1964 (Japan, copyright University of Washington) and b) landslide in La Conchita 1995 (USA, copyright U.S. Geological Survey).

What can happen if buildings are build on not compact sand? For example, if there is a strong earthquake, the soil will be liquified [2]. Houses built on wet sand sink into the ground as is illustrated in Figure 2 a) (Japan, copyright University of Washington). During the agitation due to an earthquake sand is liquified and simultaneously increases its packing density. After the earthquake the ground solidifies again so that buildings which are tilted become fixed in their new positions. 
A similar liquefaction occurs during land slides [3]. An illustration of a landslide which occurred at La Conchita, as shown in Figure 2 b) (USA, copyright U.S. Geological Survey). A road was build on a mountain, which extends from the lower left to the upper right in the image. A landslide then dislocated the road, but did not move it as a block. It is visible in the image that the deformation of the road is parabolic. This occurred because the soil was liquified by the landslide and behaved like a pasty liquid, in which the velocity is slow at the boundaries and high in the middle. This static to dynamic transition plays an important role in nature and will also play an important role in this thesis. 



\section{Chapter 1}

\section{Physical Background}

\subsection{Sand types and sand phenomena}

Sand is a very common material in the world. Most people have played with sand at the beach or in a sand box so almost such everybody has experiences with that kind of granular matter.

There exists a rich variety of sand phenomena like e.g quick sand. It was reported from travels in deserts and is still topic of science [4]. Lohse and coworkers studied the dry quick sand [5], which consists of fine grains with a very low packing density $\rho_{\text {packing }}=0.4$. A big particle will simply fall through the pile.

In deserts only $3 \%$ of the ground are covered by sand and wind can build sand dunes. The grain size of sand dunes are sorted by wind because small grains are blown away and large grains stay at their place [6]. A heavy storm can emerge as a sand storm in which fine sand can be carried more than $1000 \mathrm{~km}$, which frequently happens in the Sahara [7]. Another phenomena is 'singing sand' [8]. Its sound is created by the collective avalanche of the sand and can be very loud. A larger grain size results in a lower sound frequency.

A further phenomena is the liquefaction during landslides or earthquakes in terms of a solid fluid transition as mentioned in the introduction.

Inside a granulate friction between the grains and force chains (chain like force network with arches of the grains) plays an important role and was studied by many scientists, e.g. Refs [9, 10, 11, 12, 13, 14]. Geng et al. studied the footprints in sand and looked how the force network in a granulate is distributed [15]. 
The size of the grains has a large influence on the named phenomena. For example water drains fast through large grains without filtering the water, whereas small grains can filter it to get drinking water. The size fraction of the grains can be reduced by environmental effects like wind which can sort the grain size in dunes or like the waves of a river or in the sea. The size of the grains was rationed in size fractions by Krumbein and Sloss [16] and is shown in Table 1.1

\begin{tabular}{|c|c|}
\hline fraction name & size [mm] \\
\hline \hline colloid (mud) & $<0.001$ \\
clay (mud) & $<0.004$ \\
slit (mud) & $0.004-0.0625$ \\
very fine sand & $0.067-0.125$ \\
fine sand & $0.125-0.250$ \\
medium sand & $0.25-0.50$ \\
coarse sand & $0.5-1.0$ \\
very coarse sand & $1-2$ \\
very fine gravel & $2-4$ \\
fine gravel (granule) & $4-8$ \\
medium gravel (pebble) & $8-16$ \\
\hline
\end{tabular}

Table 1.1: Size fraction of grains labeled by Krumbein and Sloss [16].

The grain size of sand and beads used in this thesis starts from very fine sand with $80 \mu \mathrm{m}$ diameter, over fine sand, medium sand and coarse sand with a diameter of $1.1 \mathrm{~mm}$.

But not only the size influences the phenomena of a granulate. Material and shape of the grains are also important. A sand castle made of glass beads is not as stable as one of rough sand of the same size. The shape of the sand grains depends on the location where the sand was found. River sand close to mountains contains sharp edged sand whereas beach sand is normally round. That sand was rounded due to the continuous motion of the waves. Sand typically consists of quartz, chalk, limestone, heavy minerals, and shell fragments.

In Figure 1.1 a collection of optical microscopy images from different sand types sieved to the same size fraction of coarse sand is shown. The four types of sands found on four continents are a) round Ottawa quartz sand from the river Ottawa (Canada), b) sand from a sand pitch in Göttingen (Germany) consisting of sand stone, c) edged quartz sand from a stone desert (Egypt), and d) Emu Bay chalk sand (Australia) consisting of shell fragments. 

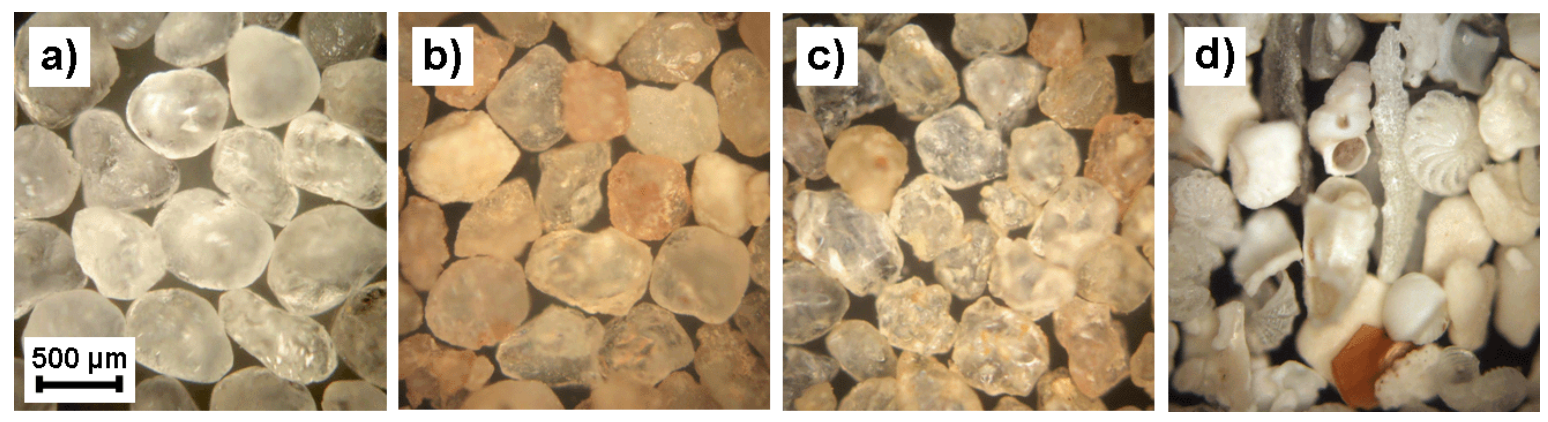

Figure 1.1: Small representation of the huge variety of different sand types. a) Round Ottawa quartz sand, b) sand from a sand pitch in Göttingen consisting of sand stone, c) edged Egypt quartz sand from a stone desert and d) Emu Bay sand consisting of shell fragments.

Scientist want to use a more regular granulate than sand. They want to apply models to their findings which works much better with beads. In this thesis mostly glass beads were used.

A crystal of monodisperse beads in a face centered cubic packing (FCC) or hexagonal close packing $(\mathrm{HCP})[17]$ have the largest packing density $\rho_{\text {crystal }}=0.74$. A lower packing density $\rho_{\text {packing }}=0.68$ can be found if the crystal has defects. At $\rho_{\text {packing }}=0.65$ only local crystals were found and Anikeenko et al. observed a fivefold symmetry in hard sphere packing [18].

The maximal random bead packing density, the so called random close packing $\rho_{R C P}=0.64$ (RCP), was verified by by many scientists, e.g. Refs [19, 20, 21]. In experiment this high density is normally not reached due to friction between the beads. The packing density of the beads can approach the maximal value, if the grains are disturbed by agitating or tapping, which was investigated by Knight et al., Xu et al., and Richard et al. [22, 23, 24]. Chen et al. increased the packing density of glass beads by thermal cycling [25].

A lower packing density, the so called random loose packing (RLP) will be reached if you can exclude gravity. The value for RLP is $\rho_{R L P}=0.55$ at zero gravitation force. It was experimentally explored by Onoda and Liniger by sediment density matched glass beads [26]. Shapiro and Probstein measured the RLP in a suspension of hard spheres with viscosity measurements and found $\rho_{R L P}=0.52$ [27].

If dry beads are filled into a container, you will often find a packing density $\rho_{\text {packing }}=0.60$. Friction between the beads prevents them of reaching the RCP, whereas gravitation prevents the beads of reaching the RLP by collapsing to loose packing. 
But what if the particles are not beads, e.g., cylinders or ellipsoids? The cylinder packing was studied by Andersson and Keeffe [28]. They found for a body centered cubic cylinder packing $\rho_{\text {packing }}=0.68$ and for the densest hexagonal cylinder packing $\rho_{\text {packing }}=0.91$.

The experimentally measured coordination number of randomly distributed cylinders rises from six at a aspect ratio (length / diameter) of 1 to 10 for large aspect ratios. It was derived by Blouwolff and Fraden [29].

The compaction of cylinder packing during agitation was analyzed by Villarruel at al. [30] and compaction of not spherical articles was done from Zou and Yu [31].

Donev et al. [32] looked at ellipsoids and showed that a maximally random jammed state of M\&Ms chocolate candies has a packing density of about $\rho_{\text {packing }}=0.68$, which is $4 \%$ larger than random sphere packing [32]. Furthermore, Donev et al. showed in 2004 via computer simulations random packings of other ellipsoid aspect ratios and packing densities approaching that of the densest sphere packings, i.e. filling nearly $\rho_{\text {packing }}=0.74$ of space at a coordination number of ten.

There are four relevant forces in wet granular matter: the force due to weight of the grains, capillary force, electrical force, and van der Waals force. Their value heavily depends on the size of the grains. We will compare their absolute value on different bead radii to find the important force in our bead size range from $40-1100 \mu \mathrm{m}$.

At very small length scale (nm) van der Waals force plays an important role and the slope is $R^{-6}$. The Hamaker constants vary a lot with different materials and the values are typically $\pm 10^{-19} \mathrm{~J}$. Silicone oxide of glass beads has a Hamaker constant of $6 \cdot 10^{-20} \mathrm{~J}$ [33]. Our smallest bead radii are larger than $40 \mu \mathrm{m}$ and we can surely neglect this force for the grains.

The force due to weight of a bead is:

$$
F_{g}=m g=\frac{4}{3} \pi R^{3} \rho_{\text {mass }} g
$$

$m$ is the mass of the beads, $\rho_{\text {mass }}$ is the density of the bead material, and $g$ is the acceleration due to gravity.

The capillary force is attractive for a wetting liquid and can be written as $F=2 \pi R \gamma \cos \theta$ cf. Eq. 1.6 [34]. Capillary force is normally small in the macroscopic world, but on small length scales it becomes the dominant force over other forces [35, 36]. This is because the capillary force decreases linearly with the bead radius whereas the force due to weight scales 
down by the third power of the bead radius [37].

The capillary force is equal to the forces of weight when a bead sitting below a cover slide and a capillary bridge (cf. Figure 1.4) holds the bead. The critical radius of the bead, when the capillary bridge can not hold the bead any more, is obtained by balancing Eq. 1.6 and Eq. 1.1

$$
R_{c}=\sqrt{\frac{3 \gamma \cdot \cos (\theta)}{2 \rho_{\text {mass }} g}}
$$

The length scale is $R_{c}=2.0 \mathrm{~mm}$ for glass beads. This value is close to the capillary length $R_{\text {droplet }}=3.2 \mathrm{~mm}$ of water, if you assume a droplet of water instead of a bead.

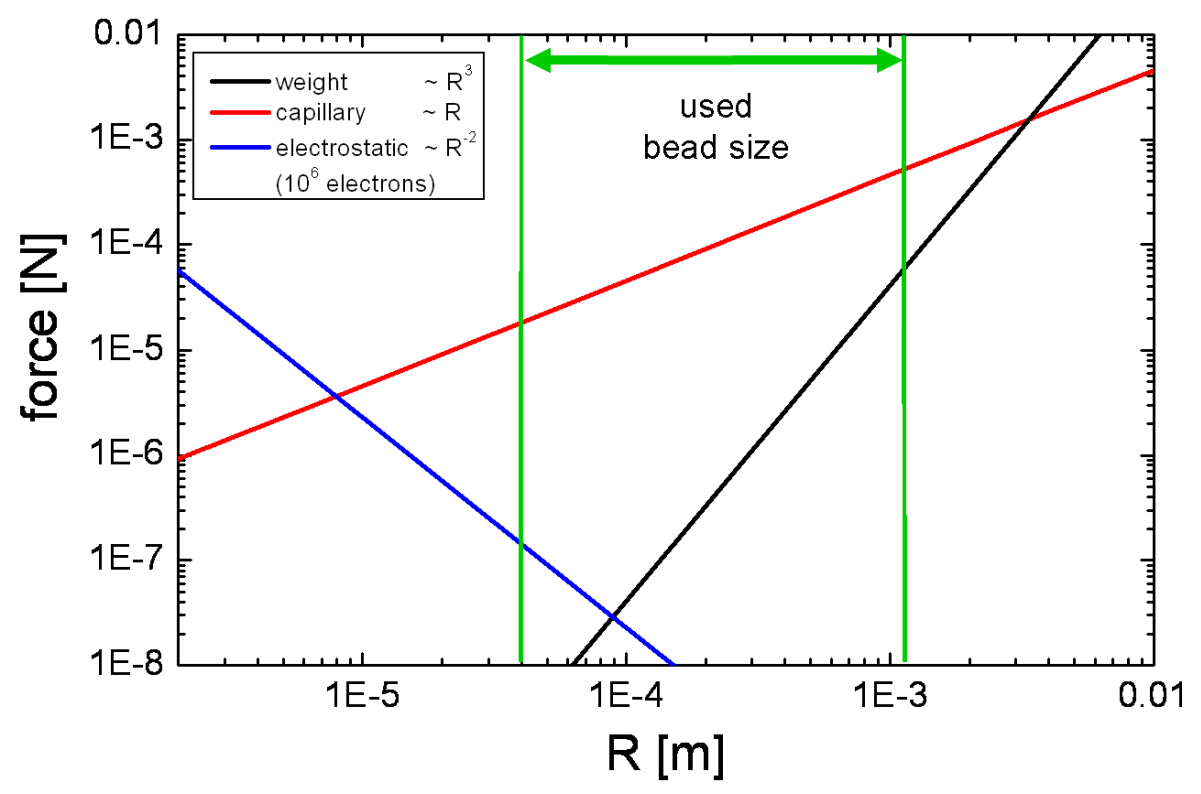

Figure 1.2: Comparison of the force values at varied bead radii. The force due to the weight of one bead is shown as black line, the capillary force of a wetting liquid between two beads in contact is shown as red line, and the electrostatic force with a charge of $10^{6}$ electrons between two beads in contact is shown as blue line.

For a complete picture we consider the values of the electrostatic force. In Figure 1.2 we have assumed the charge of $10^{6}$ electrons siting at the center of two beads. The assumed charge is strong enough to hold glass beads on a vertical plastic wall as was experimentally observed. The force can be calculated from following equation:

$$
F_{e l}=\frac{1}{4 \pi \epsilon_{0}} \cdot \frac{q^{2}}{R^{2}}
$$

$q$ is the charge of a bead and $\epsilon_{0}$ is the permittivity of free space. The electrostatic force plays 
a role only in dry experiments. In wet systems with an electrically conductive liquid we can surely neglect electrostatic force.

In the macroscopic world the force due to the weight of the grains is dominant as shown in Figure 1.2. The crossover to the capillary force occurs in the millimeter range. In our bead range from 40 to $1100 \mu \mathrm{m}$ the capillary force dominates all other forces. The effects of the liquid in a bead pile are the focus of this thesis. Therefore in the next paragraph the capillary force is discussed in more detail.

\subsection{Wetting on a substrate}

In this section capillarity phenomenon of a fluid on a solid substrate will be discussed. The capillarity allows a wetting liquid to rise into a thin tube to minimize its surface energy. This is a result of unbalanced interfacial energies of the liquid (liquid / vapor, liquid / solid, and solid / air interface). It results in a contact angle from the liquid with the substrate. The next paragraph first focuses on the liquid effects on a plane substrate and then between beads.

\subsubsection{Surface tension, contact angle and Young's law}

Liquid molecules in the bulk are surrounded by molecules of their own kind and are pulled equally in all directions, which results in a zero force on the molecule. In contrast molecules on the surface are pulled only inside the liquid and are less attracted by a second medium like air. Therefore all surface molecules create a sum of an inward directed force. This phenomenon is known as surface tension. The surface tension ' $\gamma$ ' is defined as the excess free energy per unit area at the liquid-air interface. If there are two phases SV (solid/vapor), LS (liquid / solid) and LV (liquid/vapor) touching each other, the surface tension is called interfacial tension $\gamma_{i j}$, where $i$ and $j$ refer to the two different phases. Interfacial tension is the general case and surface tension the special case of interfacial tension with the second 'liquid' being air [33].

If the liquid surface is curved, the pressure inside and outside of the liquid will be different due to the surface tension. This pressure difference is called Laplace pressure and is given by:

$$
\Delta P=P_{\text {inside }}-P_{\text {outside }}=2 H \gamma=\left(c_{\|}+c_{\perp}\right) \gamma
$$


$H$ is the mean curvature of the curved interface and can be calculated from the mean of the two local principal curvatures $\left(c_{\|}\right.$and $\left.c_{\perp}\right)$ in perpendicular directions:

a)

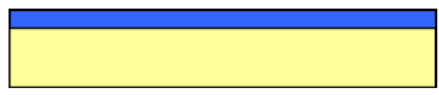

$\theta=0^{\circ}$

complete wetting b)

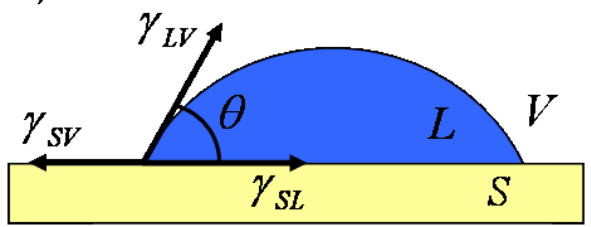

$0^{\circ}<\theta<180^{\circ}$

partial wetting c)
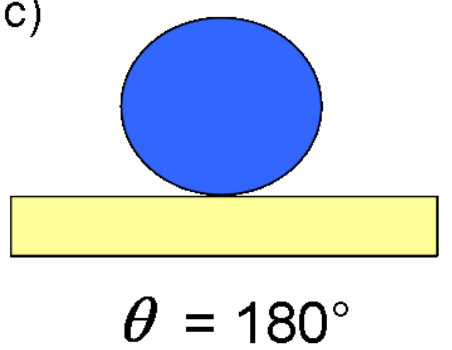

non - wetting

Figure 1.3: Schematic of a droplet on a plain surface. a) complete wetting, for $\theta=0^{\circ}, \mathrm{b}$ ) partial wetting for $0^{\circ}<\theta<180^{\circ}$ and c) non-wetting for $\theta=180^{\circ}$. S, L and $\mathrm{V}$ denotes the solid, liquid and vapor phase.

When a liquid is placed on a solid surface, the liquid droplet will form a contact angle at the three phase contact line between the LV and the LS interface. In equilibrium surface energies of the three phase contact line of a droplet is balanced and all horizontal components of the surface tensions $\gamma_{L V}, \gamma_{S L}$ and $\gamma_{S V}$ have a tendency to minimize their surface area $[33,38,39,40,41,42]$. This can be written in a form which is known as Young-Dupré equation (Eq. 1.5] [39, 43].

$$
\gamma_{S V}-\gamma_{S L}=\gamma_{L V} \cdot \cos \theta
$$

Figure 1.3 shows a schematic of the three wetting cases. A wetting liquid has a contact angle of zero and spreads on the substrate over long distances as sketched in a). In between both extremes, wetting and non wetting regime, there will be a contact angle in the interval $0^{\circ}<\theta<180^{\circ}$. This is known as the partial wetting and the liquid has a finite LS interfacial area as shown in Figure $1.3 \mathrm{~b}$ ). If all liquid remains as a spherical droplet on the surface, then the liquid do not wet the surface and the maximal contact angle $\theta$ is $180^{\circ}$ and this case is shown in Figure $1.3 \mathrm{c}$ ).

In experiments pinning effects of the contact line are observed and the receding contact angle can be much smaller than the Young contact angle and the advancing contact angle can be larger than the Young contact angle [38]. 


\subsubsection{Capillary force between two beads}

When liquid is placed between two beads in contact then a toroidal ring structure is formed. If the liquid wets the beads, an attractive force will hold the beads together. This force plays an important role in nature, when grains become wet. It was investigated by many scientists, e.g. Refs [44, 45, 34, 46, 47].

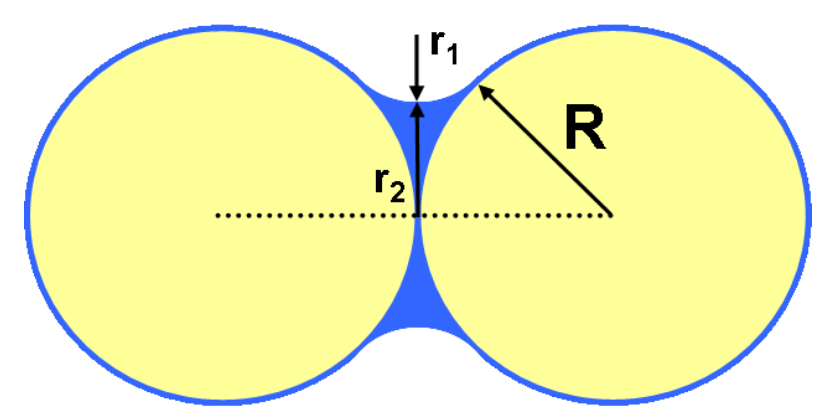

Figure 1.4: Schematic representation of a capillary bridge between two beads in contact.

In the illustration of Figure 1.4 is $R$ the bead radius, $r_{1}$ is the radius of curvature of the capillary bridge, and $r_{2}$ the radius of the capillary bridge. For beads in contact the capillary force is given by [34]:

$$
F=2 \pi R \gamma \cos \theta
$$

The decrease of the capillary force as a function of the contact angle $\theta$ was experimentally verified by Garoff et al. [48] and calculation was done by Schubert [49]. A similar formula for the capillary bridge force was found by Cross et al. [50].

In Figure 1.5 a) a capillary bridge between two beads with separation distance $S$ and the half opening angle $\beta$ is shown. The capillary force of a capillary bridge with a contact angle $\theta=0^{\circ}$ decreases rapidly with an increased distance [34] and is shown in Figure $1.5 \mathrm{~b}$ ).

At the rupture distance $S_{c}$ the capillary bridge ruptures. A new capillary bridge can only be formed, if two beads touch each other. Lian et al. [47] reviewed a functional dependence of the critical rupture distance:

$$
S_{c}^{*}=V^{* 1 / 3} \cdot\left(1+\frac{\theta}{2}\right)
$$

$V^{*}=V / R^{3}$ is the normalized volume of the capillary bridge. The rupture distance scales with 

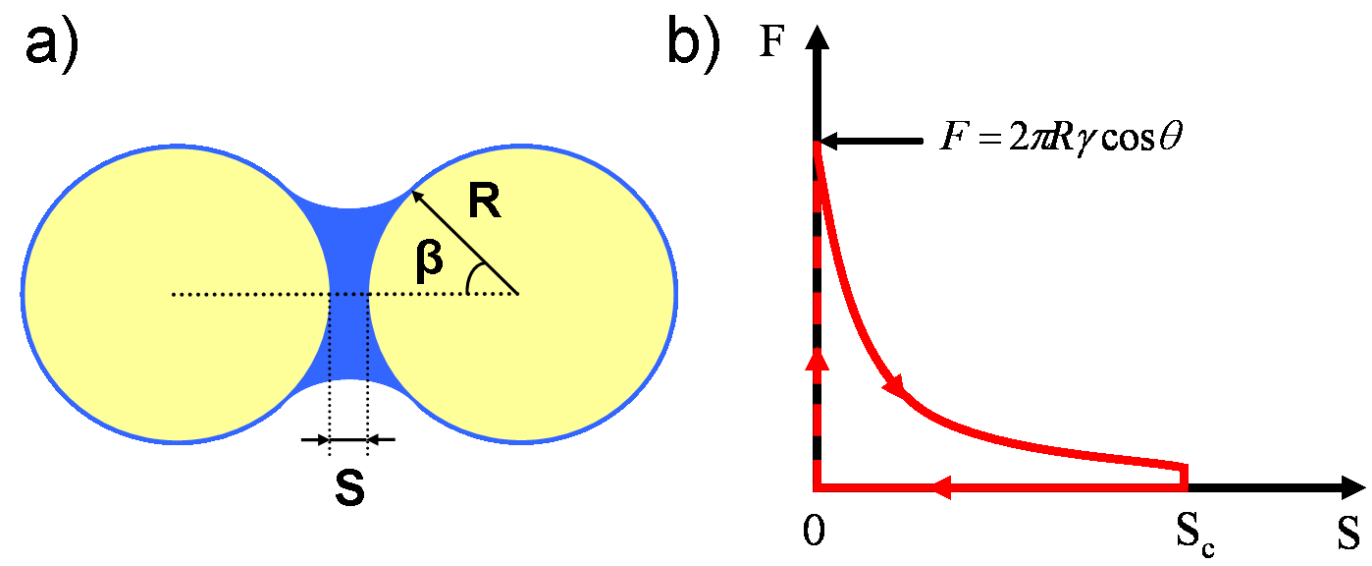

Figure 1.5: a) Schematic representation of a capillary bridge between two beads with separation distance $S$ and a half opening angle $\beta$ of the capillary bridge. b) The capillary force decays with increased separation distances.

the third root of the liquid volume. The rupture distance is very important in the case that mechanical manipulation will break capillary bridges, like the tensile strength measurements in chapter 3.

The capillary force equation as a function of the separation distance

$$
F=\frac{2 \pi R \gamma \cos \theta}{1+1.05 s^{+}+2.5 s^{+2}}
$$

is valid up to an half opening angle $\beta<10^{\circ}$ (with an accuracy of better than $4 \%$ ). The dimensionless separation is $S^{+}=S^{*} / \sqrt{V^{*}}$ whereas $S^{*}=S / R$ the bead radius normalized separation distance between the beads is. In a random ordered pile of beads some capillary bridges have an increased separation distance $S^{*}$. Or if any process increases the separation distance between the beads like shearing or fluidization, the capillary force decreases.

\subsubsection{Wetting from low to large liquid contents $W$}

The liquid content $W$ in a wet granulate is defined by the liquid volume divided by the total sample volume ( $\left.W=V_{\text {liquid }} / V_{\text {total }}\right)$. It can range from $W=0$ to $W=0.4$ and the maximal liquid content depends on the packing density $\left(W=1-\rho_{\text {packing }}\right)$. The capillary force between two beads depends on the volume of the capillary bridge and was theoretically investigated by Halsey and Levine [51]. 
a) asperity regime

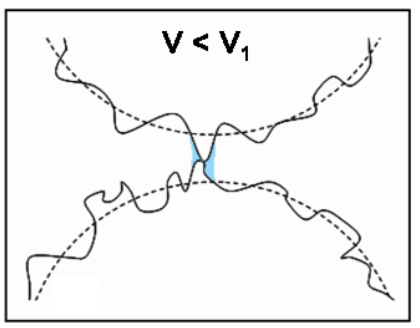

b) roughness regime

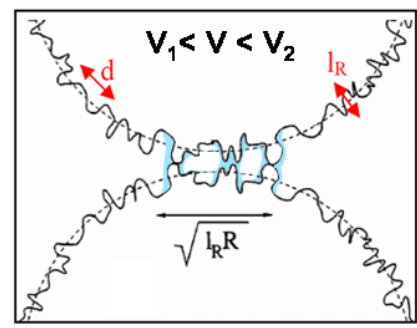

c) spherical regime

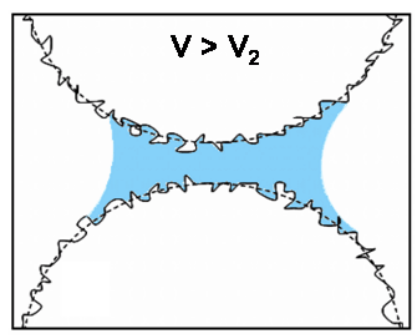

d)

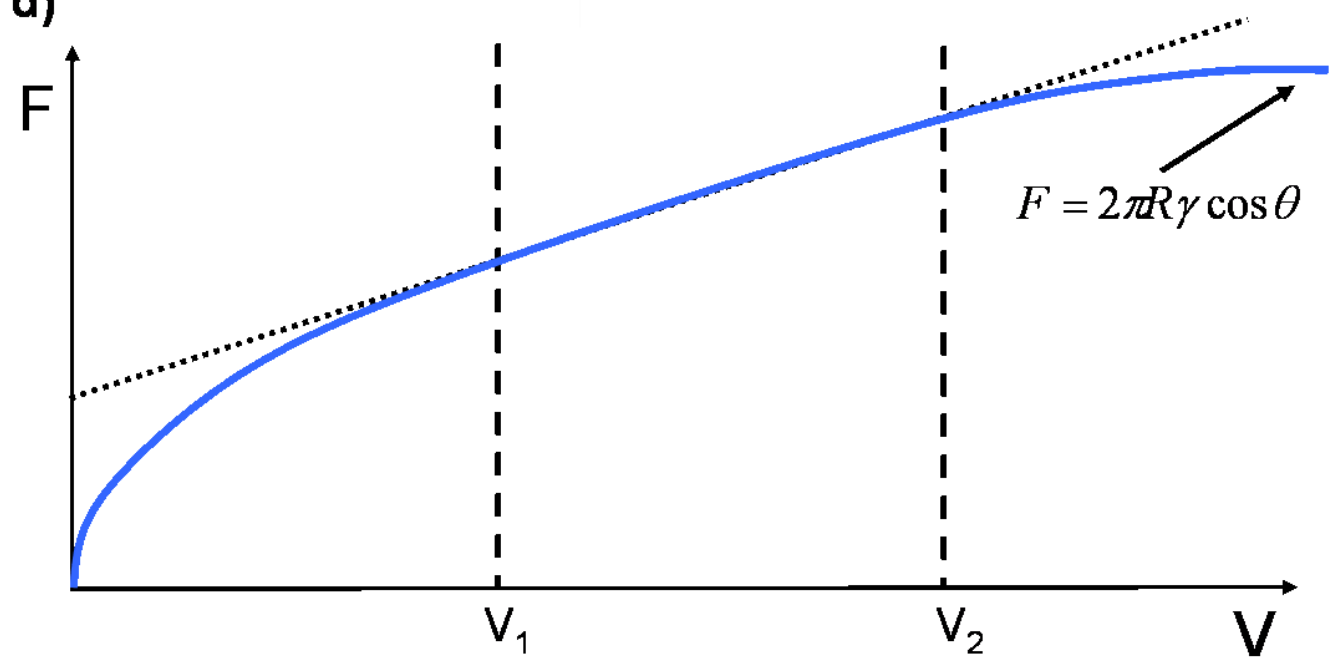

Figure 1.6: Three wetting regimes are illustrated in a) asperity, b) roughness, and c) spherical. d) Shows how the force scales at the three wetting regimes.

First, we start with the dry case and increase the liquid content. Micro bridges between point contact are developed on peaks of the surface roughness. This liquid content is named asperity regime in which micro bridges have a very small volume. A schematic of the asperity regime is shown in Figure 1.6 a). Experimental images of micro bridges can be found in Weeks et al. [52] and Schenk et al. [53]. The liquid content of the asperity regime of our glass beads is very small with about $W<10^{-5}$. Samples in this regime can be prepared by controlling the humidity of the air.

With more liquid many micro bridges around the same point contacts are created as shown in Figure $1.6 \mathrm{~b}$ ). This regime is called roughness regime and the liquid content for our glass beads ranges normally from $W=1-10 \cdot 10^{-5}$ and depends on the surface roughness. Samples can be prepared as well with condensing liquid out of air. 
With even more liquid in the pile the spherical regime is reached, where a 'complete' capillary bridge is formed cf. Figure $1.6 \mathrm{c}$ ). This regime has a liquid content larger than $W>10^{-4}$ for our glass beads. Samples can be prepared by mixing the liquid with the grains, because the humidity of the air is not important any more at such large liquid contents.

Figure 1.6 d) shows the force between the rough beads as a function of the liquid volume in all three wetting regimes [51]. In the asperity regime the force scales with cube root of the liquid volume $V^{1 / 2}$. In the roughness regime the force scales linear with the liquid volume $V$. In the spherical regime the force is nearly independent from its volume $V$. The capillary force stays constant for more than two decades of liquid contents [34, 51]. With increased opening angle $\beta$ of the capillary bridge the capillary force decreases slowly and reaches at $\beta=30^{\circ}$ a $20 \%$ smaller value [54] as the maximal capillary force of Eq. 1.6 .

Experiments in the full range of liquid contents were performed by Feng and Yu [55]. They measured the packing density of beads and observed a packing density $\rho_{\text {packing }}=0.46$ for beads $R=125 \mu \mathrm{m}$, which is a smaller packing density than the RLP. This is possible since the capillary force holds loose beads structures stable which collapse in the dry case.

Harris and Morrow [56] drained acid through a pile of steel beads and measured from the area of the etched beads the Laplace pressure of the filled pores and capillary bridges. The Laplace pressure ranges from large absolute Laplace pressures at low saturation to 5 (normalized by surface tension and bead radius) at larger liquid contents. This was in agreement with direct measurements of the Laplace pressure of capillary bridges done by Fischer [57].

An overview of the physics of wet granulates is given by Herminghaus [58] and Mitarai et al. [59]. In this thesis the full range of liquid contents is used. The focus is normally in the spherical regimes at a liquid content $W=0.005$ to the completely submerged case $W=0.4$. The more complicated liquid content of $W>0.025$, in which capillary bridges merge into larger liquid morphologies, will be discussed in chapter 4 . 



\section{Chapter 2}

\section{Microscopic measurement techniques}

In this chapter we characterize our system, namely the roughness of the beads and the contact angle of the liquid on the beads. The roughness of the beads made from glass, basalt, and ruby was measured by AFM (atomic force microscopy). The contact angle of water on these surfaces was determined by optical microscopy. Furthermore, we introduce the X-ray micro tomography, which allowed us to image the 3D-liquid distribution in a wet pile of beads. Along this line necessary processing steps to extract the information of this raw data are explained as well.

\subsection{Surface roughness measurement of the grains}

The mechanical properties of wet granulates are significantly affected by the capillary forces of the liquid and friction between the grains. The friction and the contact angle are sensitive to the surface roughness of the particles. Since different bead diameters were used in the mechanical characterization experiments and the measured results should be compared we have characterized the surface roughness as a function of the bead radius.

The topography of the beads surfaces were measured using an AFM Nanoscope V Dimension 3100 from Veeco Instruments $\mathrm{GmbH}$ in Tapping Mode ${ }^{\circledR}$. Typically a $10 \mu \mathrm{m}$ by 10 $\mu \mathrm{m}$ large area of the grain surface was scanned. Figure 2.1 a) shows a few typical AFM topography images for different bead materials. The topographic images of glass beads bought from Whitehouse Scientific Ltd (UK) have textured surfaces with up to $1 \mu \mathrm{m}$ large objets embedded in the smooth surface. Some batches are completely covered by many $100 \mathrm{~nm}$ large objects. These particles could not be removed by cleaning the beads with ethanol and 

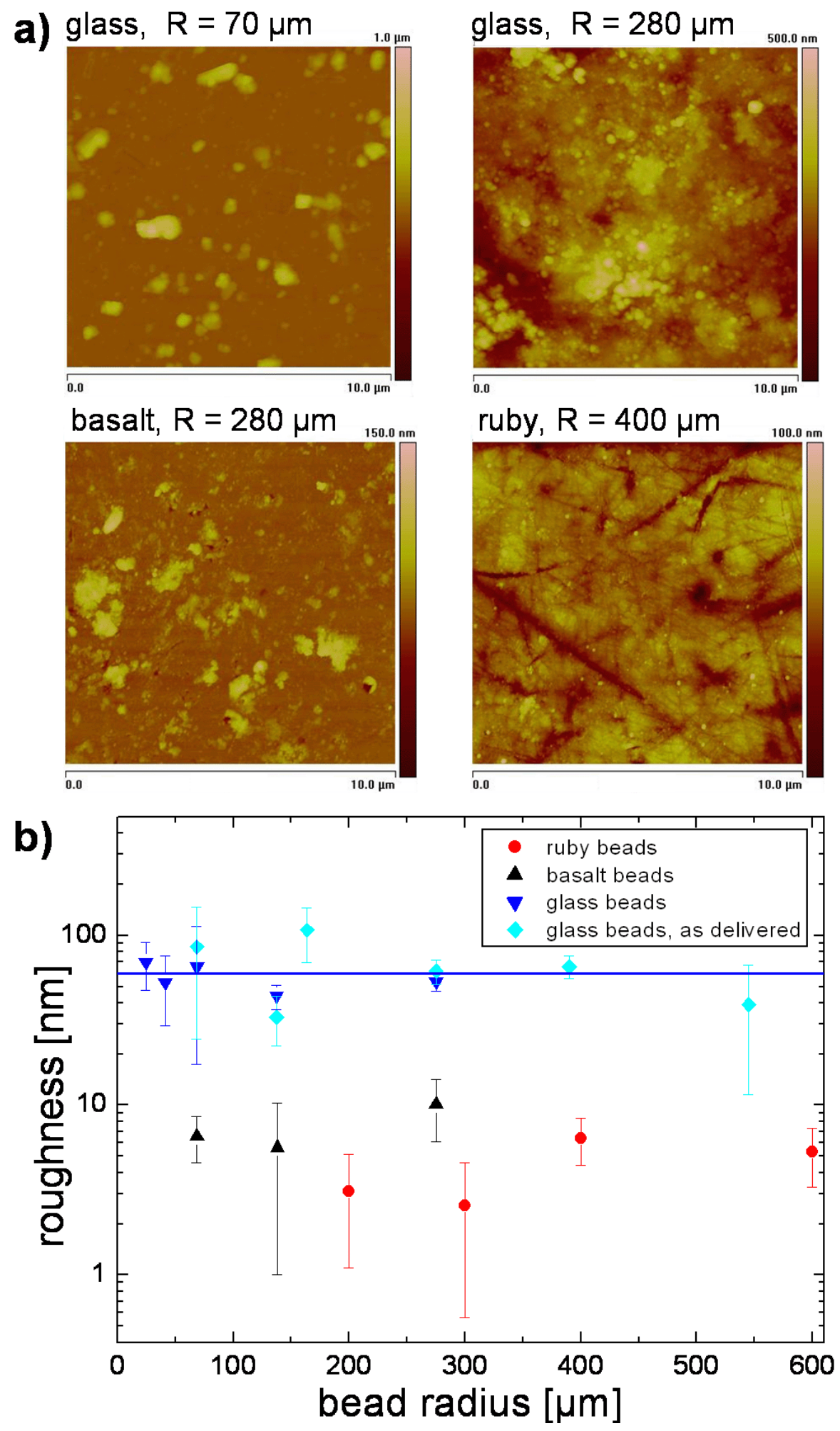

Figure 2.1: a) Topographic AFM images of the different used beads. b) RMS roughness derived from several AFM measurements for different bead sizes and bead materials. 
Milipore $^{\circledR}$ water. The basalt beads bought by Whitehouse Scientific Ltd (UK) have a much smoother surface than glass beads. On the bead surfaces only some particles of up to $100 \mathrm{~nm}$ size were found. The ruby beads were produced and polished from Saphirwerk Industrieprodukte $A G(\mathrm{CH})$ and Sandoz Fils $S A(\mathrm{CH})$. We could barely find particles on the bead surfaces and we found only many scratches from the polishing process.

For the measurement of the root-mean-squared roughness (RMS) the curvature of the beads was corrected with a second order plane fit and more than three beads were analyzed. The results for glass beads, ruby beads and basalt beads are shown in Figure $2.1 \mathrm{~b}$ ). The given error is the statistical error of several measurements. The glass beads have a roughness of about $60 \mathrm{~nm}$ and the roughness does neither depend on the bead diameter nor the cleaning. Basalt and ruby beads surfaces are smooth. Basalt beads have a RMS roughness of about $8 \mathrm{~nm}$ whereas ruby beads have $4 \mathrm{~nm}$; no size dependence was found.

In conclusion we found no systematic size dependence of the RMS roughness for all measured beads. The roughness depends only on the manufacture method how the beads were produced. The basalt and ruby beads have an one decade smaller RMS roughness than glass beads.

\subsection{Contact angle and Laplace pressure measurement}

The attractive force of the liquid morphologies in a granular pile strongly depends on the surface tension and the contact angle of the liquid with the bead surface. The surface tension is determined by the used liquid and the contact angle can be measured from capillary bridges in a granular pile. Varying the bead material results in different contact angles of the liquid with the beads. The Laplace pressure can be determined by both radii the curvature of the capillary bridge $r_{1}$ and radius $r_{2}$ of the capillary bridge, cf. Eq. 1.4. The technics used for the contact angle and Laplace pressure measurement will be explained below.

\section{Contact angle measurement of capillary bridges between beads}

The contact angle hysteresis determined by measuring the receding and advancing contact angle of a liquid on a substrate. We used a Leica microscope DM4000 B in transmission mode and a Leica DFC350 FX camera with a pixel resolution of $0.6 \mu \mathrm{m}$. Two ruby beads were placed in contact on a microscope slide. The liquid was injected with a micro capillary of about $10 \mu \mathrm{m}$ produced with a Sutter Model P-97 from Sutter Instrument Company and we 
removed the micro capillary for an undisturbed system. The liquid was evaporated and the surface line of a capillary bridge moved on the ruby beads. We show the receding angle measurement with two ruby beads in Figure 2.2. The ruby bead radius with $300 \mu \mathrm{m}$ correspond to 470 pixels. The image resolution is large enough to calculate the contact angle of the liquid from the slope in the interception point of fitted circular arcs to the respective interfaces $r_{2}$ of the capillary bridge and the bead radius $R$. The half opening angle $\beta$ can be determined from the same inception point as used for the contact angle measurement. The image analysis was done using the ImageJ software.

For the advancing contact angle measurement we placed a peltier element under the microscope slide with the beads and cooled the sample below the dew point. Water from the air condensed into the capillary bridge and the surface line moved. The analysis of the parameter works equally as for the receding measurement.

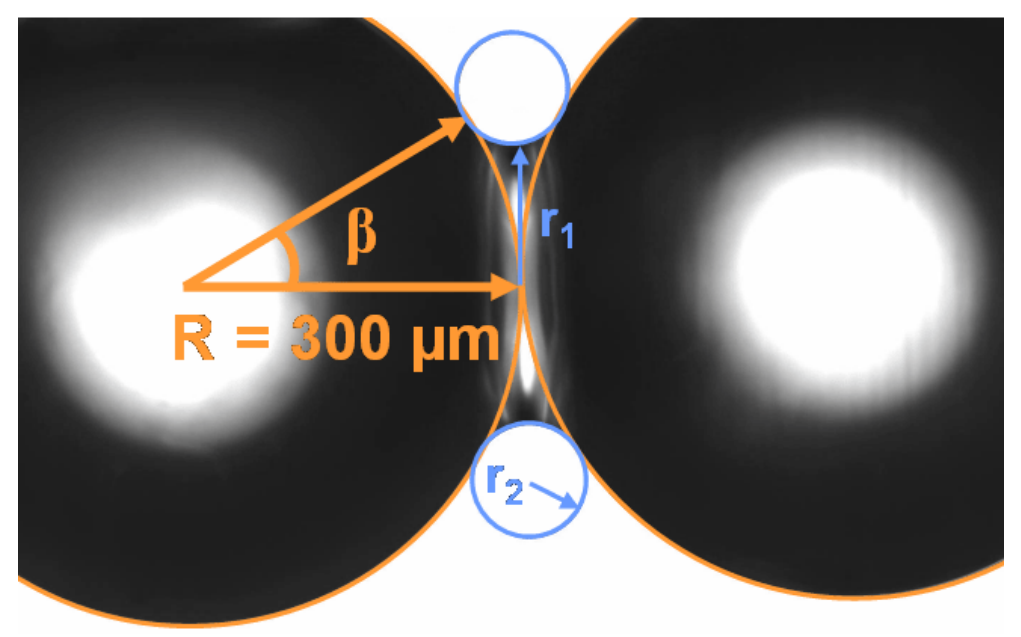

Figure 2.2: Optical micrograph as used to determine the contact angle of the liquid or the Laplace pressure of the capillary bridges. Circular arcs were fitted to the respective surfaces $r_{1}, r_{2}$, and $R$. The half opening angle $\beta$ was calculated by the interception point of the arcs and the contact angle from the slope at the interception point.

\begin{tabular}{|c|c|c|c|}
\hline material & receding angle $\theta_{\text {rec }}\left[{ }^{\circ}\right]$ & advancing angle $\theta_{\text {adv }}\left[{ }^{\circ}\right]$ & typical angle in a pile $\theta_{\text {pile }}\left[^{\circ}\right]$ \\
\hline \hline glass & $5 \pm 5$ & $40 \pm 10$ & $10 \pm 10$ \\
ruby & $10 \pm 10$ & $75 \pm 10$ & $55 \pm 10$ \\
basalt & $10 \pm 10$ & $85 \pm 10$ & $70 \pm 10$ \\
\hline
\end{tabular}

Table 2.1: Contact angle of water on the bead materials glass, ruby, and basalt. 
The measured receding angles for water on the bead materials glass, ruby and basalt are listed in Table 2.1 and show small receding angles of $\theta_{\text {rec }}=10^{\circ} \pm 10^{\circ}$. The advancing contact angles for water was found to vary in the range of $\theta_{a d v}=40^{\circ}-85^{\circ}$. The advancing contact angle varied strongly due to pinning effects on the rough surface. The large contact angle hysteresis makes it challenging to determine, which contact angle is present in a wet granular pile. Whenever individual liquid morphologies changes their volume, we expect a change of the apparent contact angle. Therefore, we measured the typical contact angle in an equilibrated wet granular pile.

\section{Measurement of the typical contact angle in a pile}

To get as close as possible to the 3D situation in a random pile of beads we enclosed a homogenously mixed wet granulate between a microcopy slide and a cover slide and sealed them by a rubber o-ring to prevent evaporation. The capillary bridges between the beads in around four layers were imaged following the same measurement method as performed before.
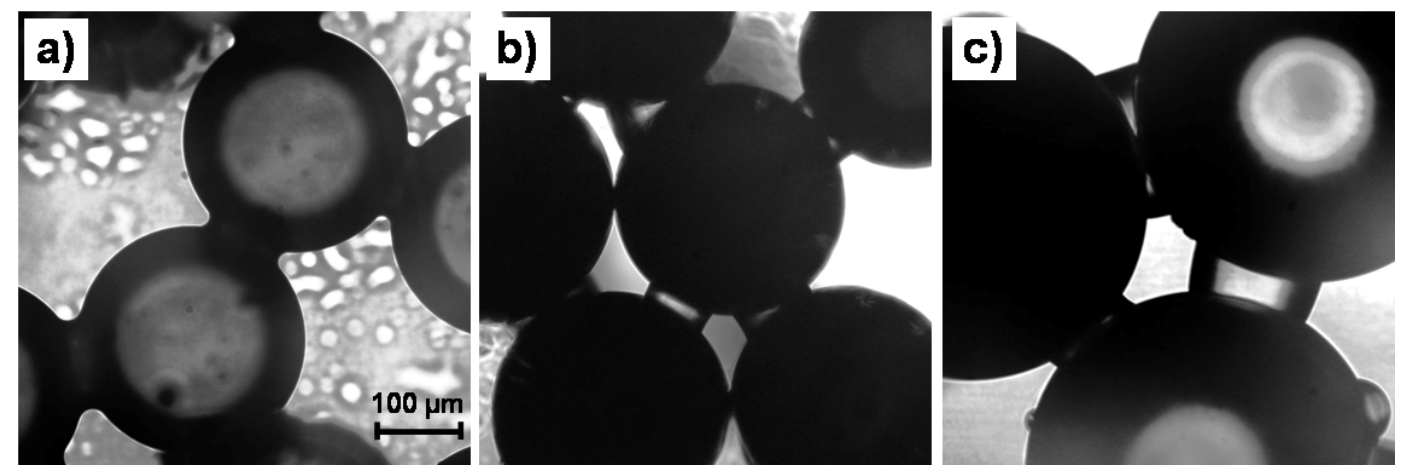

Figure 2.3: Optical microscopy images of capillary bridges in random piles of beads a) glass, b) basalt, and c) ruby. Scale bar applies to all images.

Figure 2.3 shows typical microscopy images of capillary bridges found in piles of beads from different materials: a) for glass beads, b) for basalt beads, and c) ruby beads. To obtain a reproducible result for the contact angles we averaged over more than 10 individual measurements. As liquid we used water for all beads. Typical contact angles are listed in Table 2.1 For glass beads we found a typical contact angle $\theta_{\text {pile }}=10^{\circ} \pm 10^{\circ}$. For ruby beads we obtained $\theta_{\text {pile }}=55^{\circ} \pm 10^{\circ}$ and with basalt beads we measured $\theta_{\text {pile }}=70^{\circ} \pm 10^{\circ}$.

Since the capillary bridge force scales with $\cos \theta$, the different contact angles expect to influence the mechanical properties significantly. As for the stiffness of a wet granulate we expect only $57 \%$ for ruby beads and $34 \%$ for basalt beads compared with the value of glass beads. 


\section{Laplace pressure measurement}

The measured parameters of capillary bridges from the contact angle measurement allowed us to calculate the Laplace pressure (LP) inside a capillary bridge. Variation in the symmetry due to gravitation can be neglected [34]. The LP in Figure 2.4 is normalized by the bead radius and the surface tension $\gamma$. For the Laplace pressure measurements of liquid morphologies the liquid was evaporated and condensed onto the beads in an open system to the air. These measurements allow for comparison with numerical minimizations done by Martin Brinkmann [60] using the Surface Evolver [61].

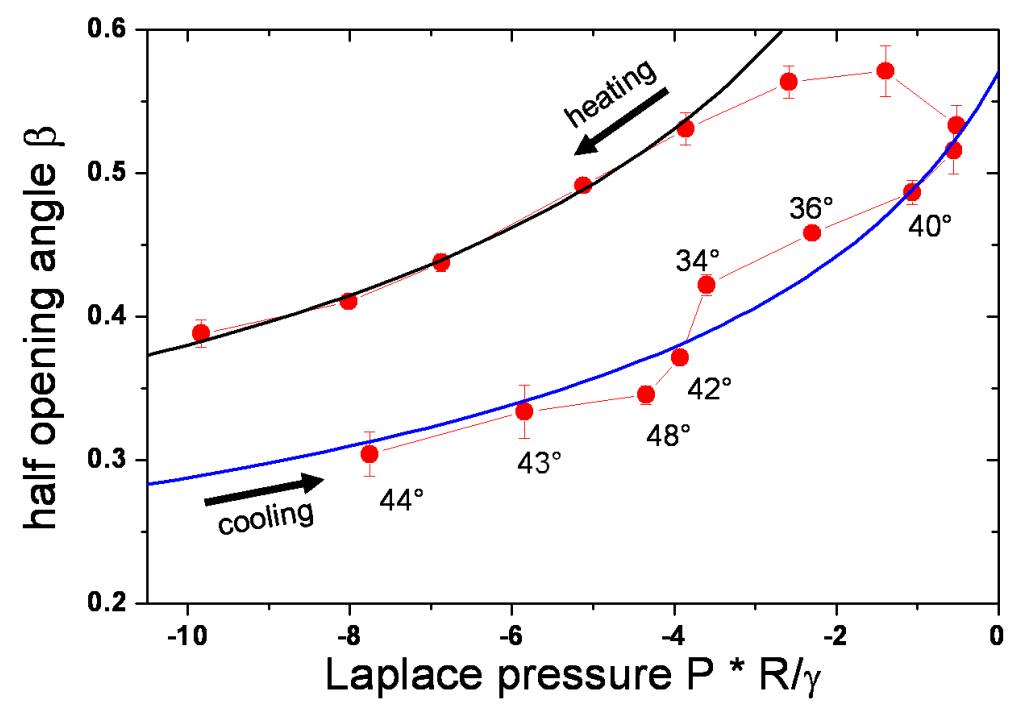

Figure 2.4: By heating and cooling the sample the volume of capillary bridges can be controlled. The graph shows part of a hysteresis loop from a condensing and evaporating capillary bridge. Its data points (red dots) are in good agreement with the numerical minimizations curve for $40^{\circ}$ (blue line) [60]. In the evaporating case the values follow the curve for $5^{\circ}$ (black line) of a receding contact angle.

By placing the microscope slide on a peltier element we were able to cool and heat the sample between temperatures of $10^{\circ} \mathrm{C}-50^{\circ} \mathrm{C}$. For these measurements we condensed liquid out of the air humidity into a capillary bridge and evaporated liquid from the same capillary bridge. The liquid used for the capillary bridge was a salt solution $\left(\mathrm{ZnI}_{2}+\right.$ surfactant $)$. It was also used for the X-ray micro tomography (cf. Table 4.1). The surface tension and the contact angle is slightly increased compared to water. We made a movie of the capillary bridge and split the movie into single images using the software GIOTTO 2.12. The image analysis was identical to that described with the contact angle measurements. The advancing contact angle 
of the condensing capillary bridge in Figure 2.4 for the ruby beads is around $44^{\circ} \pm 4^{\circ}$. During the condensing process pinning effects are visible and if the surface line starts to move the contact angle drops from $48^{\circ}$ to $34^{\circ}$.

When the capillary bridge reached a large volume we started to heat the sample. The liquid evaporated, so the capillary bridge volume decreased. The receding contact angle drop to a value close to $5^{\circ}$ and the contact line moved smoothly over the surface.

We found that the Laplace pressure of a capillary bridge can be compared very well with results obtained by numerical minimizations. The small variations of the experimental data are a result of the contact line pinning, which is strongly visible for the advancing contact angle.

\subsection{X-ray micro tomography}

The 3D distribution of a wetting liquid in a random pile of glass beads, a three phase system: grains, liquid, and air, can be determined using various techniques. One can use confocal microscopy, magnetic resonance imaging (MRI), neutron-tomography or X-ray micro tomography. Optical microscopy is limited on scattered light of impurities in the glass beads and on variation of the refraction index of the grains, liquid and air phase. A good image can be obtained only for the first surface layers close to the container walls. This turns out to be a fundamental drawback, since the packing geometry of the beads is altered close to the wall. Since we are interested in the liquid distribution between random distributed grains, these limitations of the optical index matching techniques prevent us from having good statistics and to image larger liquid clusters. MRI and neutron-tomography typically have a limited lateral resolution above $0.1 \mathrm{~mm}$ and quite long data acquisition times. To guarantee that the typical length scale of our liquid morphologies are well below the capillary length of around $3 \mathrm{~mm}$ and to surely neglect gravitation effects on the liquid distribution for our liquids we need a high resolution in the $\mu \mathrm{m}$ range. For good statistics we also need large samples and a good temporal resolution to capture slow dynamic processes like the equilibration of liquid as we will discuss in chapter 4 . The technique which satisfies all these requirements is the X-ray micro tomography that was used to capture the 3D packing geometry and the liquid distribution in wet granulates [62]. Due to the different X-ray absorption contrast of the three phases: liquid, beads, and air the three phases can be clearly distinguished from each other by using a salt liquid. 
All the X-ray micro tomography measurements presented in this thesis were done at the synchrotron ESRF (European synchrotron radiation facility) in Grenoble (France). The high energy beamline ID15A has a high speed micro tomography setup, which can obtain a full tomography in some seconds and has a resolution down to about $2 \mu \mathrm{m}$ [63]. Since 2008 the imaging speed was improved even further, so that a full tomography can be recorded in less than 0.5 seconds, provided that the sample can withstand the high X-ray flux. The schematic tomography setup is shown in Figure 2.5. To capture a full 3D image typically $7502 \mathrm{D}$ absorption images were collected while continuously rotating the sample by $180^{\circ}$. The minimum number of projections depends on the pixel size and the size of the sample. From the set of projections a 3D image was calculated by a standard filtered back-projection algorithm. This calculated 3D image we refer to as a raw image. It was used to determine the structural information of our wet granular piles using further image processing.

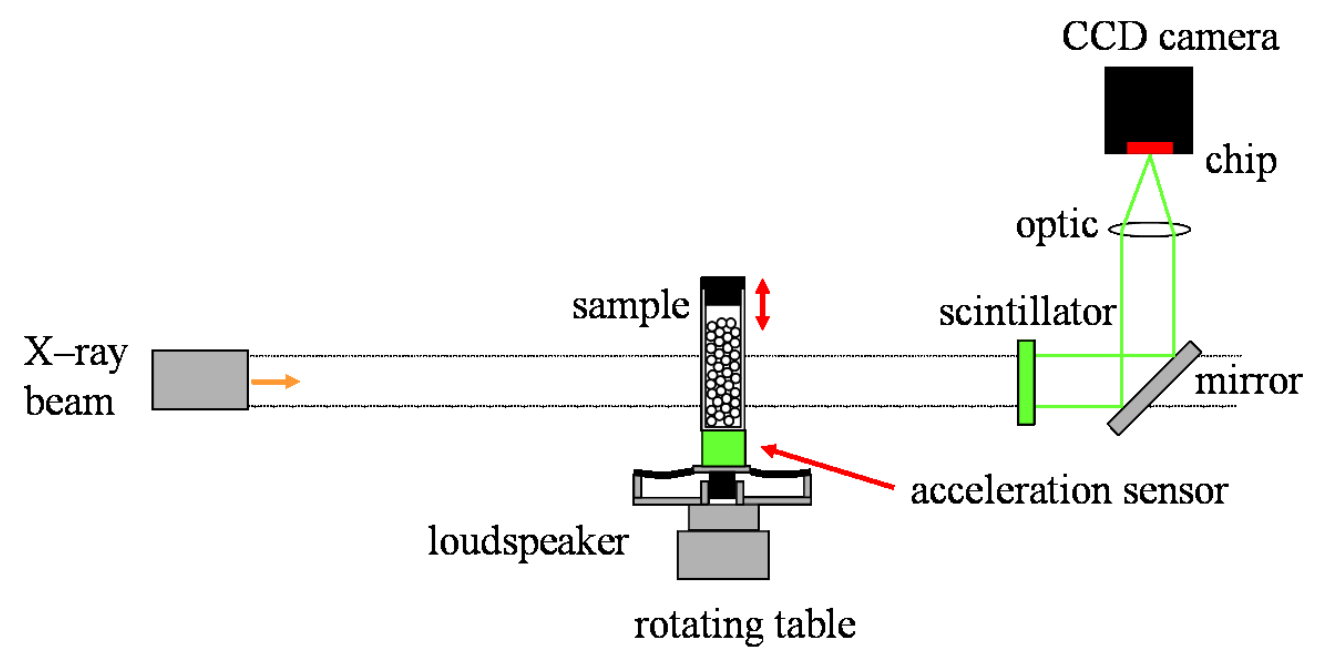

Figure 2.5: Illustration of a tomography setup at the beamline ID15A (ESRF).

The source of the X-ray beam was a wiggler, in which the electrons of the storage ring are bent by strong magnets. Due to the large storage ring (circumference $844.4 \mathrm{~m}$ ) the generated bremsstrahlung has an excellent collimation. The X-ray beam used for the tomography experiments has a peak energy of around $40 \mathrm{keV}$. The intensity of the X-ray beam is adapted to our experiment by passing rods of PMMA to prevent our samples from radiation damage. The transmitted part can be evaluated by absorption law $I=I_{0} \cdot \exp (-k \cdot d) . I_{0}$ is the initial intensity of the beam, $d$ is the thickness of the material and $k$ is a material constant which depends on the beam energy. 
The photon energy is large enough to pass some centimeter thick samples without being completely absorbed. The X-ray photons can be absorbed of a material by photo effect, compton scattering and pair production. The photo effect, where electrons are kicked out of the electron sheath, is the dominant effect in the energy range used. Pair production can be completely excluded at our used photon energies, since an energy larger than $1.022 \mathrm{MeV}$ would be required. The absorption coefficient $k$ increases with the third power of the electron number of the atoms. This explains why air, which contains mostly nitrogen with 7 electrons, has a fairly low X-ray absorbtion. Glass beads or sand grains contain mainly $\mathrm{SiO}_{2}$. Si-atoms have 14 electrons. As well the larger mass density must be taken into account. The iodine used in the aqueous salt solution has 53 electrons in the electron sheath. This leads to a larger absorption of the liquid than with glass beads. By varying the salt concentration we can adjust the absorption contrast. The transmitted X-ray intensity of the sample is converted into visible light by a scintillator. We used a YAG:Ce with a thickness of $300 \mu \mathrm{m}$ and $600 \mu \mathrm{m}$. More light signal gave a LuAG:Ce scintillator with $1 \mathrm{~mm}$ thickness as it was available 2008. The light is typically collected by a 1x magnifying macro lens onto a CCD-camera Dalsa Dalstar 1 M60 or a Sarnoff CAM512. The combination of the used optic and pixel size of the CCD-chip defines the resolution which was typically $14 \mu \mathrm{m}$ (Dalstar) or $18 \mu \mathrm{m}$ (Sarnoff) per pixel. For a very high resolution a magnifying lens was used leading $2 \mu \mathrm{m}$ resolution.

The lateral size of a tomography image was limited in width by the size of the CCD sensor to $14 \mathrm{~mm}$ and the height was limited by the extension of the X-ray beam to $5.5 \mathrm{~mm}$. The vertical profile of the X-ray beam had an intensity like a Gaussian with cut edges. Close to the edges of the beam profile the intensity was low, resulting in a poor signal to noise ratios, which in some cases prevented us from analyzing the full image height. The maximal intensity in the image was chosen close to saturation of the CCD sensor. To reduce the vertical intensity variation of the beam profile, we placed rods with a diameter of $8 \mathrm{~mm}$ of alumina and PMMA into the beam. Due to the differences in the material thickness, the X-ray beam has to penetrate the rods and is more absorbed in the middle than at the edges. With all the absorbing material present the beam intensity was about three times lower. That could be compensated by opening the wiggler gab to almost saturation of the pixels of the CCD sensor for an expose time of $2 \mathrm{~ms}$. Shaping the beam intensity by the rods, the lateral variation of the X-ray intensity was significatively reduced and the signal to noise ratio was almost constant in one 3D image, thus enabling us to analyze the full image. Minor intensity variations of the raw image was normalized slice by slice the same glass absorption value using Matlab 8.5.1. 

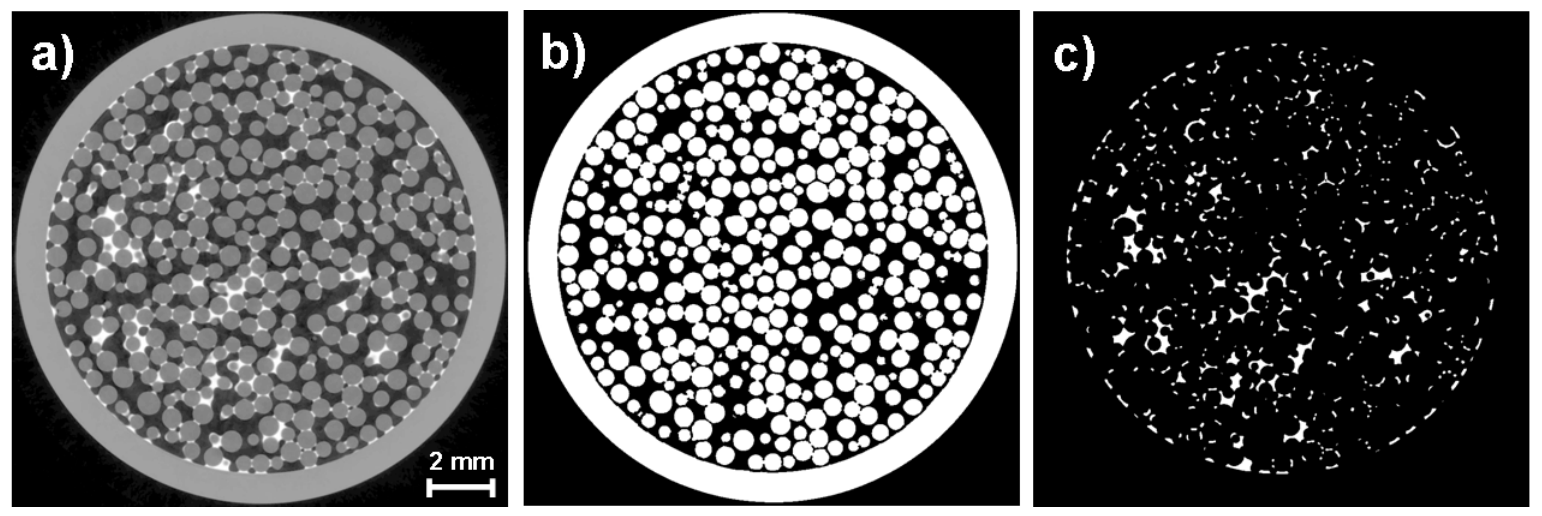

Figure 2.6: a) Horizontal slice of a 3D image of wet bead pile. b) Segmented image of the grain phase with the glass container of a). c) Segmented image of the liquid phase of a).

A horizontal slice through a 3D tomogram is shown in Figure 2.6 a). The three phases of grains, liquid and air can be clearly distinguished. The beads have a diameter of $550 \mu \mathrm{m}$ and the liquid content was $W=0.07$. The polydispersity of the beads was about $10 \%$ (of the mean radius), sufficient to prevent crystallization. The slice cuts the beads at different heights and thus the beads appear to be more polydisperse than they actually are. The color scale in Figure 2.6 a) is chosen such that a higher X-ray absorption corresponds to a brighter color. The liquid we used was an aqueous salt solution with $\mathrm{ZnI}_{2}$. The X-ray absorption for the liquid phase was adjusted to be larger than the absorption of the glass beads, which allowed the detection of even small liquid features.

To numerically extract the structural information from the 3D raw tomography images, they needed further postprocessing. For a quantitative analysis of the tomography images the raw data had to be smoothed and segmented. Since typical 3D noise reduction algorithms usually do not lead to a satisfying result or significantly broaden the edges, a 3D anisotropicdiffusion (AD) filter and three phase segmentation of the software packages 'Mango' by Sheppard et al. [64] were used. The three phase segmentation algorithm [65] uses a seed method, in which a threshold level is selected for the well defined 'areas' (low, intermediate and hight phase) and the algorithm increases these 'areas' [66]. Three binary black and white images were created, in which either the beads, the liquid or the air phases appear as white and everything else is displaced as black. In Figure 2.6 b) the segmented beads are displayed and in c) it is the liquid. The segmented images have been used for all further numerical data analysis, of which an overview is presented now. 


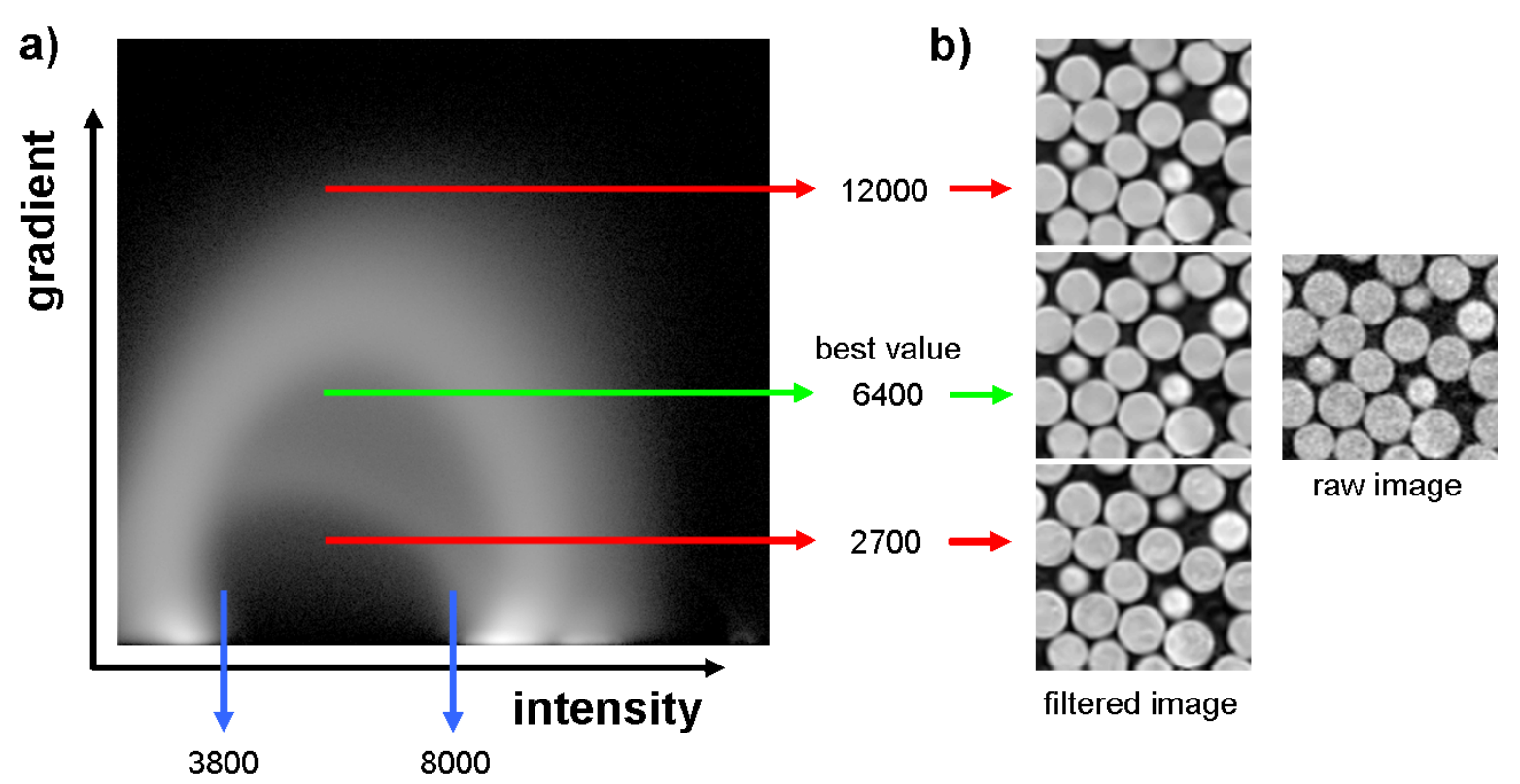

Figure 2.7: a) The intensity gradient histogram of a tomography image shows the intensity of the voxels on the $\mathrm{X}$-axis and the gradient on Y-axis. The pixel intensity reflects how often this gradient and intensity appear in a voxel of the tomography image. The air phase and grain phase are visible as bright 'areas' connected by a loop. Thresholds of the intensity and the gradient are used for filtering and segmenting the image. b) Example of filtered images at different gradient thresholds. A to large gradient thresholds (top image) remove fine image details and a too small gradient (bottom image) filter less of the noise. Only a right gradient value (middle image) results in good images for the further processing. The raw image is shown for comparing the improved image quality. 
The Mango program contains over 100 possible input parameters. In the following discussion only the relevant parts of the image analysis will be described. Furthermore, only important parameters of the image analysis will be mentioned. After the precorrection with Matlab 8.5.1 we loaded the image with Mango and started the processing by creating an intensity gradient plot from the tomography image as shown in Figure 2.7 a). In this histogram the $\mathrm{X}$-axis shows the voxel intensity and the Y-axis the gradient to the surrounding voxels, whereas the brightness reflects how often this voxel appears in the 3D tomography image. In the image two bright 'areas' are visible at the bottom which are connected by a loop. The left 'area' is the air phase and the right 'area' is the grain phase. The loop shows the gradient of the connection voxels between the phases. The gradient of the tomography image is used as input parameter for the anisotropic-diffusion filter [64]. On the example of a two phase image, shown in Figure 2.7 b), it will be explained how the needed parameter can be extracted. Normally the best value is a little bit lower than the loop height. A too low value like 2700 will result in a noisy image after filtering with AD filter. A too high value like 12000 will remove important fine image details. The right value of 6400 will remove noise without smearing fine details. The raw image is shown for comparing the improved image quality.

The best gradient value smoothed image can be segmented by a 2 or 3 phase segmentation. The segmentation [65] works using a seed method [66] and the 3 phase segmentation has 18 tunable parameters. The important parameters are the four intensity thresholds for the three phases and the two gradient thresholds. After segmentation 3 black and white images remain: the low phase, the intermediate phase, and high phase. In our samples the high phase only represents the liquid with a value of 1 (white) and the rest of the image has a value of 0 (black). The intermediate phase only shows the grains with the value 1 . In the low phase the pore space is 0 . All the further image processing starts after the segmentation. 


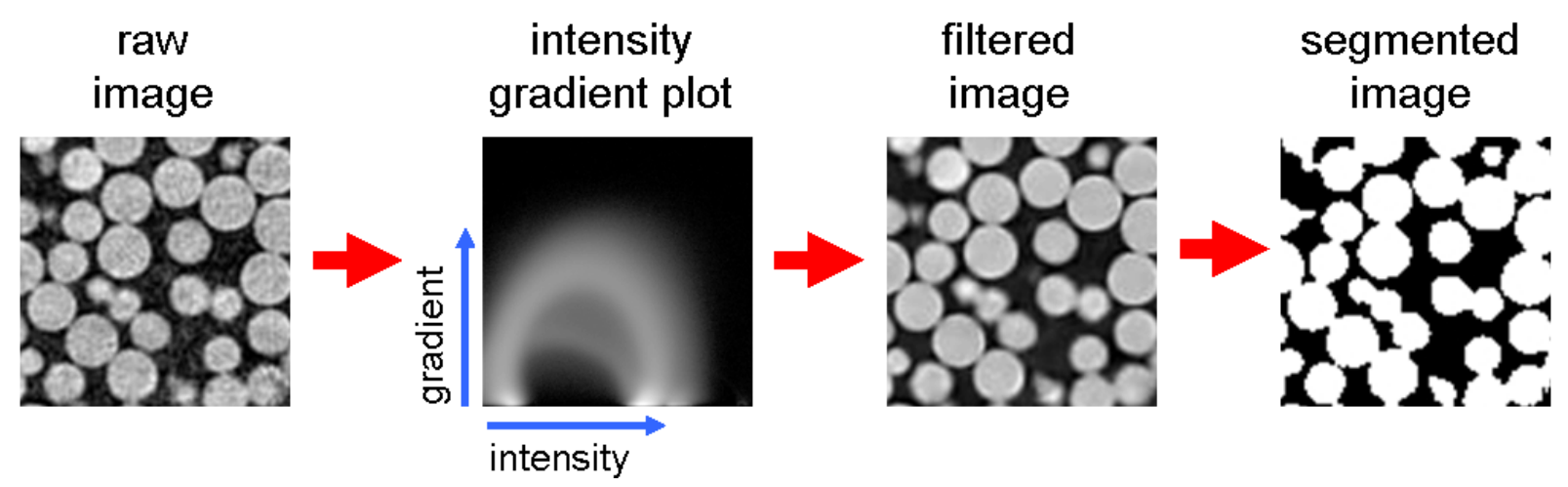

Figure 2.8: The first steps of the image processing up to the segmentation with Mango software [64].

The first processing steps using Mango are shown in Figure 2.8 for a 2 phase tomography image. From a raw image the intensity gradient histogram was calculated and the intensity and gradient threshold was extracted. Then the image was filtered with AD filter and segmented in air and grain phase, of which the grain phase is shown in the last picture in Figure 2.8.

\section{Euclidean distance}
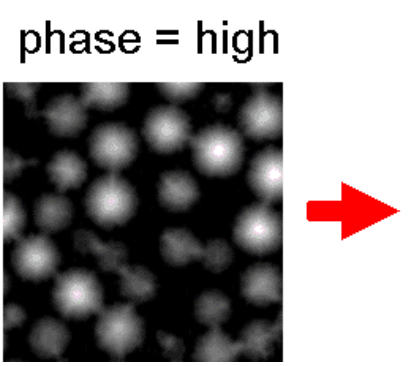

\section{particles}

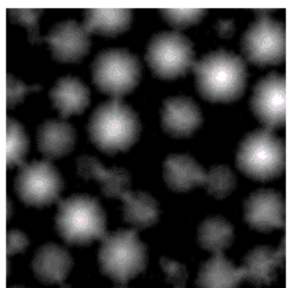

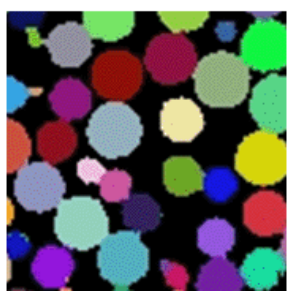

Wigner Seitz cell

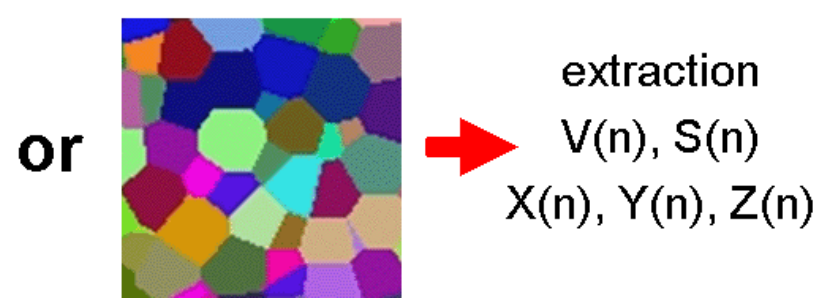

Figure 2.9: The beads can be separated by using an Euclidean distance map, that allows to detect the single particles or the Wigner Seitz cell. It also allows extraction of the volume, surface, and position of each bead.

The additional image processing steps using segmented images. From the grain phase we extracted the volume, the surface, and position of all beads as shown in Figure 2.9. This was not trivial, because all of the particles were connected and they needed to be separated. A very careful choice of the parameters used was mandatory. This was done by creating an Euclidian distance map which labels the distance to the phase border using the intensity of the image. The brightness peaks in the distance map label the centers of the grains and allow the watershed filter by increasing these 'areas' to separate all beads from each other. Holes, which might be present in a glass bead, pose a challenge to these algorithm since they divide 
the beads into small zones. The individual beads can now be labelled with different grey scale or color values and be exported to an image. Instead of using the watershed algorithm, the Euclidean Voronoi tessellation can be used to extract the Wigner Seitz cell around the particles. The high phase of an image corresponds to the liquid in the granular pile and can be directly labelled by a grey scale and exported. This is not shown in an extra image.

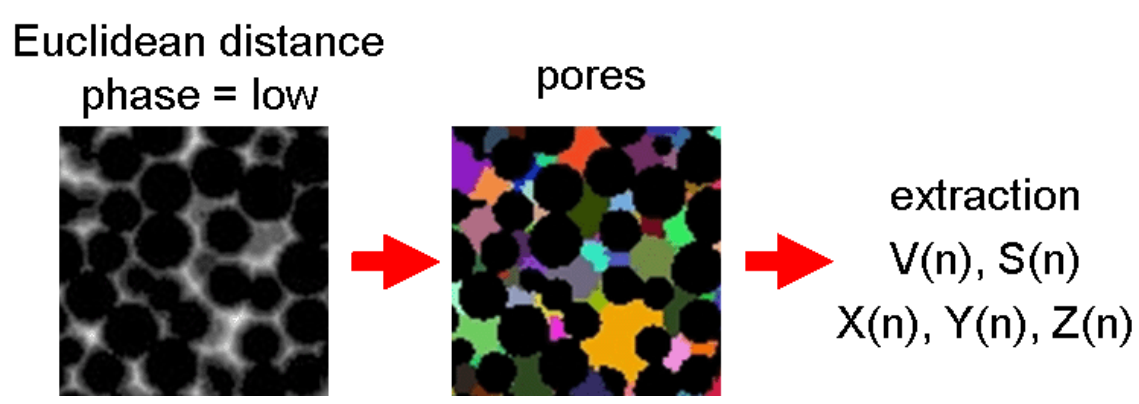

Figure 2.10: The percolated pores can be separated in small pore cells by using an Euclidean distance map.

The next processing steps focuses on the intermediate phase as illustrated in Figure 2.10. This time the distance map is created from the low phase in the image. All pores are connected and have to be separated by the watershed algorithm, so the parameters such as the size or position can be extracted. At the end of the processing all information can be stored in separate images and text files. This data can further be processed by Matlab 8.5.1 for example by extracting connectivity lists between the grains or between the grains and the liquid.

\section{Packing density of a wet bead pile measured by X-ray micro tomography}

With the analysis discussed above the packing density of the grains in a pile can be determined. The packing density has an influence on the mechanical behavior of the granulate. If the granulate is densely packed, the material has an increased stiffness since on average every grain has a larger number of nearest neighbors. This leads to a larger number of capillary bridges which increases the stiffness as discussed in chapter 4 . If the material density is known, the packing density can be measured by weighing the grains and measuring the volume $\rho_{\text {packing }}=m / \rho_{\text {mass }} V$ where $V$ denotes the total sample volume. One additional source of error is the ordering induced by the container wall which will affect the packing density, if the surface to volume ratio of the container is too large.

To account for this surface effect, we only analyzed the bulk volume of our X-ray micro tomography images and disregarded volumes which were closer than 5 bead diameters to the 
container walls. Since different packing densities are possible, we prepared our samples with two methods. For method 1 the sample was strongly shaken. After agitation the sample was given a strong tap to the bottom and then an additional cap was pressed on top. For method 2 the sample was prepared as before without pressing a cap.

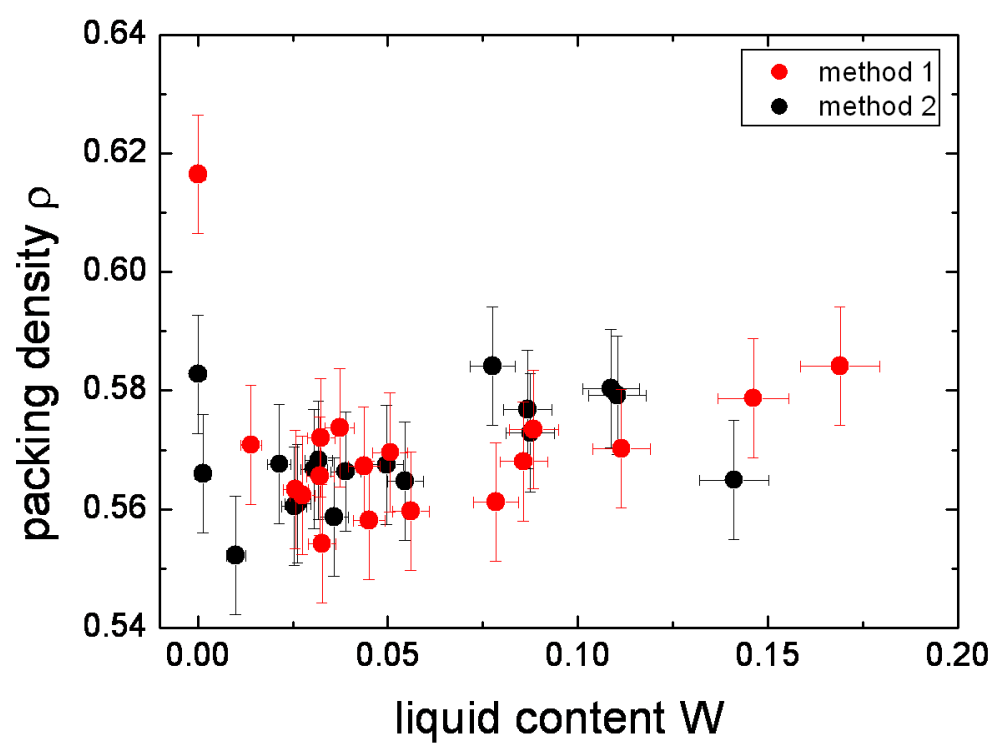

Figure 2.11: The packing density of glass beads $R=140 \mu \mathrm{m}$ as a function of the liquid content for two preparation methods obtained by X-ray micro tomography.

Figure 2.11 shows the packing density obtained from the tomography images for both methods with glass beads $R=140 \mu \mathrm{m}$ as a function of the liquid content. Both preparation methods result in a very similar packing density. Only the dry data point obtained with method 1 at $\rho_{\text {packing }}=0.62$ has a slightly larger packing density. For wet samples both methods have a packing density $\rho_{\text {packing }}=0.57 \pm 0.01$ and show a slight increase of 0.01 with increasing liquid content to $W=0.2$. The prepared packing densities are nearly independent of the different preparation techniques and turned out to be very robust, as long as the sample was not tapped for a long time in order to increase the packing density. Thus for most of the analysis presented here we assume a packing density of $\rho_{\text {packing }}=0.57 \pm 0.01$. A main reason for the lower wet packing density compared to the dry case is that the friction (adhesion) between the grains is increased by the capillary force of both the capillary bridges and the liquid morphologies discussed in chapter 4. During the sample preparation the beads stick together and can not move into a closer configurations. 



\section{Chapter 3}

\section{Mechanical measurements}

The mechanical properties of wet granulates can be characterized using a wide variety of techniques due to their complexity [67]. For example, the angle of repose [68, 69, 70], stability angle in a rotating drum [71], axial compression [72], tensile strength [73, 74, 75], shear stiffness [76], and critical fluidization acceleration [58] were previously studied. Unfortunately no single characterization or measurement technique of the mechanical properties can give a complete picture of the large variety of effects present in wet granular matter. In Addition, not all of the techniques allows one to compare the macroscopic behavior of the grains with a microscopic picture. We measured the tensile strength, the shear stiffness, and the critical fluidization acceleration. Fluidization measurements can be implemented quickly and allow one to analyze many parameters of wet grains. By shearing and applying pressure on a wet pile the influence on the capillary network and the friction of the beads can be measured. The tensile strength technique induces a rupture of the pile and is very sensitive to the capillary forces. The results of the three mechanical methods are compared at the end of this chapter. 


\subsection{Critical fluidization acceleration measurements}

Fluidization of a granulate occurs when individual particles start to move relative each other due to external agitation. The agitation can be achieved by horizontal, vertical, or circular shaking of the sample or by air or liquid flow through a granulate sample. Combinations of two or more types of agitation are possible. For a fast and simple 'standard' test we focused on vertical shaking. The periodic vertical agitation is characterized by a sinusoidal amplitude $A(t)=A \sin \omega t$, where $\omega$ is the angular velocity of the oscillations, the peak amplitude $\mathrm{A}$, and the time $t$. $\omega=2 \pi v$ with the frequency $v$. The maximal acceleration value is given by $\Gamma=A \omega^{2}$ and it is obvious that a smaller acceleration than the acceleration of gravity has no effects on the grains. In this thesis the acceleration $\Gamma=A \omega^{2} / g$ is normalized with the acceleration of gravity to be dimensionless. Dry granulates fluidize at a critical acceleration $\Gamma_{C}=1.2$ [77, 78]. At a small filling height the bottom fluidization is dominant and at large filling heights the wall fluidization [58]. In this thesis large filling heights were used. With liquid present in the pile we expect an increase of the critical fluidization acceleration.

\subsubsection{Measurement technique}

For the measurement of the critical fluidization acceleration a homogenously mixed wet granulate was filled into a the measurement container. As a container we typically used glass vials with a $14 \mathrm{~mm}$ inner radius and $50 \mathrm{~mm}$ height. We filled the vial to three quarters of its height. To prevent the liquid, Milipore ${ }^{\circledR}$ water, from evaporating the vial was sealed with a cap. Only small amounts of liquid, less than $W=2 \cdot 10^{-5}\left(W=V_{\text {liquid }} / V_{\text {total }}\right)$, can evaporate into the gas phase of the sample assuming $100 \%$ saturation of the air at room temperature. After a short time when the air is saturated no further evaporation is possible. The sample was then mounted on a shaker, model V.409 from Ling Electronics (US), and the acceleration was directly recorded with an acceleration sensor 8776A50 from Kistler Instrumente GmbH as shown in Figure 3.1 a) [79]. Observation of the grains movement were made either using video cameras or directly by an observer present.

In order to obtain reproducible results a well defined protocol was used. To generate a reproducible starting condition the sample was shaken heavily at a peak acceleration of $\Gamma=20$ for ten seconds and then the agitation was turned off. From this well defined starting condition the vertical fluidization measurement was started by increasing the amplitude slowly at a 

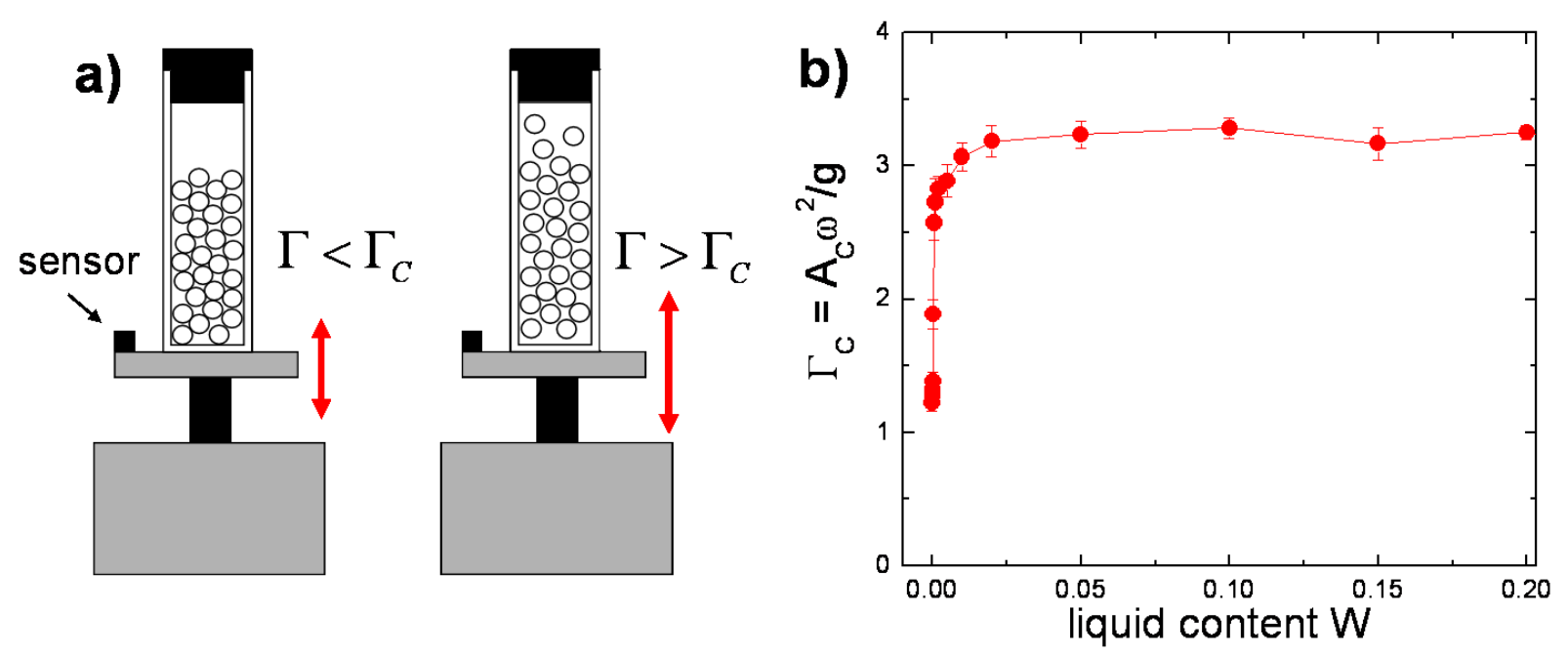

Figure 3.1: a) Schematic below and above the critical fluidization acceleration $\Gamma_{C}$ of a vertically shaken granulate inside a sample vial [79]. b) Typical dependence of $\Gamma_{C}$ for glass beads with a radius of $R=280 \mu \mathrm{m}$ on the liquid content.

constant speed. The movement of the beads was detected by observing the sample from the side at a position, which is about half of the filling height of the vial. At an agitation close to the critical fluidization acceleration the grains do not move, but some grains start to vibrate and rotate in a caged position. We determined that the critical fluidization acceleration $\Gamma_{C}$ had been reached when some grains moved more than one particle diameter relative to the surrounding grains. At $\Gamma_{C}$ you can hear the sound of moving grains. These experiments were performed for various liquid contents $W$, glass bead radii $R$, various bead materials, acceleration frequencies, and wetting liquids.

We are interested in the dependence of the critical fluidization acceleration of wet grains on the liquid content $W$. A typical result for glass beads with a radius of $R=280 \mu \mathrm{m}$ is shown in Figure $3.1 \mathrm{~b}$ ). The error is obtained from the standard deviation of five independent measurements. Starting from a dry sample, $\Gamma_{C}=1.2$, a rapid increase of $\Gamma_{C}$ is observed for increasing liquid content. At liquid contents $W \geq 0.01$ a plateau is reached with a value of about $\Gamma_{C}=3.0$ and is valid for a liquid content $W<0.20$. At large liquid contents $W>0.2$ the measurements are difficult due to drainage of the liquid, especially for larger beads, and results in an inhomogeneous liquid distribution. The detection of the critical fluidization acceleration is challenging, since the liquid includes air bubbles whose motion can be misinterpreted as movement of the grains. 
The increase of the critical fluidization acceleration at low liquid contents is a direct result of the capillary bridges exerting an attractive force between the grains as was shown by Hornbaker [80] or Halsey and Levine [51]. After the liquid content is large enough to form complete capillary bridges the plateau is reached. This can be understood by considering that the force of a capillary bridge is nearly independent of its volume over a large range of liquid volumes [34]. At liquid contents $W>0.02$ larger liquid morphologies are present and the capillary forces are more difficult to estimate.

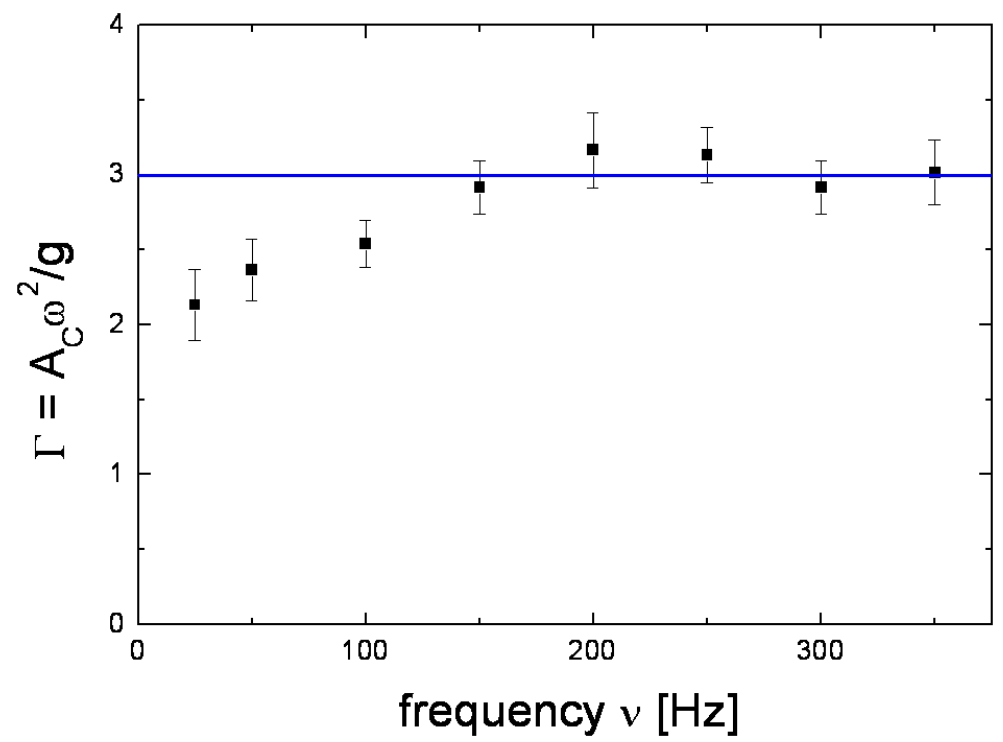

Figure 3.2: Critical fluidization acceleration of glass beads $R=280 \mu \mathrm{m}$ and a liquid content $W=0.01$ as a function of the frequency $v$.

We also tested the frequency dependence of the critical fluidization acceleration. A typical result is displayed in Figure 3.2 showing $\Gamma_{C}$ for glass beads with $R=280 \mu \mathrm{m}$ and $W=0.01$ as function of the agitation frequency. The error bars correspond to the estimated absolute error. The critical fluidization acceleration was found to be insensitive to the agitation frequency. $\Gamma_{C}$ was observed to decrease only at low frequencies. All further measurements examining the role of the liquid content $W$, bead radius $R$ or liquid properties were carried out in the plateau regime of the frequency $v=200 \mathrm{~Hz}$. 
To estimate the influence of different starting conditions we used two different measurement protocols, referred to as protocol 1 and protocol 2. Protocol 1, denoted by the black squares in Figure 3.3 a) [79], shows the sample after fluidization with a large acceleration of $\Gamma=20$. Then the agitation was turned of which led to a constant packing density of $\rho_{\text {packing }}=0.58$. Subsequently, we measured $\Gamma_{C}$ by again increasing the amplitude from zero until the critical fluidization acceleration was reached. Protocol 2, denoted as red dots in $3.3 \mathrm{a}$ ) shows the sample after fluidization and then the acceleration was decreased below the critical fluidization acceleration. To determine the $\Gamma_{C}$ the amplitude was increased until fluidization occurred.
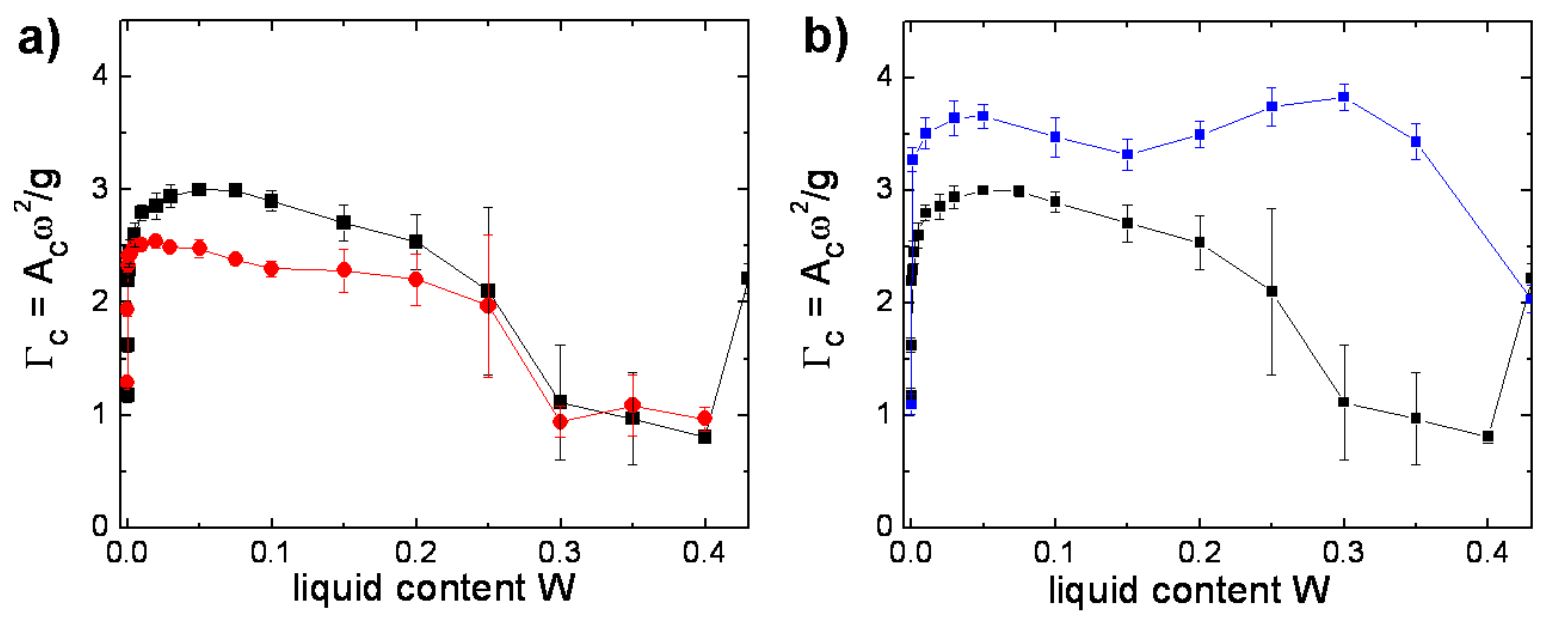

Figure 3.3: a) Dependence of the critical fluidization acceleration of glass beads of $R=240 \mu \mathrm{m}$ from the liquid content $W$ for the two measurement protocols 1 (black squares) and 2 (red dots) [79]. b) The effects of cleaning with ethanol and water on the measurement of the critical fluidization acceleration using protocol 1, cleaned (blue squares), as delivered (black squares).

In Figure 3.3 a) measurements using both protocols with varied liquid content are shown. The curve displayed is the mean of five repeated measurements. At low liquid contents $W=1 \cdot 10^{-4}$ no difference is visible to the dry case (where $\Gamma_{C}=1.2$ ) regardless of the sample history. For increasing liquid content we found a slower increase of the critical fluidization acceleration and the plateau value of about $\Gamma_{C}=3$ was found for protocol 1 and was larger than for protocol 2. Between $W=0.25$ and $W=0.4 \Gamma_{C}$ drops to 1.0. This decrease comes from trapped air bubbles, oscillating during the sinusoidal cycles at high liquid contents. In the completely submerged case $\Gamma_{C}$ is 2.2. This increase compared to the dry $\Gamma_{C}=1.2$ is a result of the viscosity of the liquid and the decreased density difference between the bead material 
and water compared to air.

Qualitatively both curves in Figure 3.3 a) show that the critical fluidization acceleration increases at low liquid contents. They both have a nearly independent value for the plateau and lower values for the completely submerged case. The plateau values differ slightly for varied measurement protocols.

From recording the sample filling height we observed that the use of protocol 2 leads to inconsistent packing densities for varied liquid contents. At low liquid contents the grains are more densely packed than with larger liquid contents. Since grains with a higher packing density have more contact points between the beads, this is expected to increase the stiffness of the wet pile for small liquid contents.

Protocol 1 results in a constant packing density, because it comes immediately to rest from a strongly fluidized state and the grains can not pack into closer configurations. Using protocol 1 we observed only a lightly larger packing density for the dry case and the completely submerged case. In order to compare the results from the fluidization measurements with all other mechanical tests and to exclude possible density variations, we restrict our discussion below to the fluidization measurements obtained using protocol 1.

We have also checked the dependence of possible contamination of the beads. All grains in this thesis were washed with ethanol and Milipore ${ }^{\circledR}$ water to remove small particles such as dust or contaminants like oil. In Figure 3.3 b) the dependence of the critical fluidization acceleration on the liquid content is plotted in comparison with beads as delivered and cleaned beads. The critical fluidization acceleration of the cleaned beads is approximately $20 \%$ larger. At liquid contents $W>0.35$ a decrease of the critical fluidization acceleration to the value of a completely submerged sample was observed. The cleaned beads show no minimum for $\Gamma_{C}$ at liquid contents $0.25<W<0.4$. There are possibly less air bubbles present in a cleaned sample. The larger values of the critical fluidization acceleration might also result from the larger surface tension of the liquid since dirt reduces the surface tension of the liquid. Thus, for the experiments discussed in this thesis, we cleaned our beads in order to have reproducible starting parameters. 


\subsubsection{Experimental results}

In this subsection measurements of the critical fluidization acceleration for wet granular piles as a function of the liquid content $W$, various liquids, and several bead radii $R$ are presented. Based on these results we derived a model to explain the experimental results. This equation should advance our knowledge in the fluidization of the grains by the wall. Wallfluidization is the dominant effect if the container diameter is small compared to the filling height. For the wall-fluidization we derived a force equation which balance the capillary bridge force and the acceleration force of the sample [81].

The force of a capillary bridge exerted between a wall and a bead $F_{\text {wall }}=4 \pi \gamma R \cos (\theta)$ was investigated by McFarlane and Tabur [82], Cross et al. [83], and Clark et al. [84]. If the two beads have a different size, it can be taken into account by their bead radii $R_{1} R_{2} /\left(R_{1}+R_{2}\right)$ [85, 86]. If a capillary bridge has one contact angle between the beads and another contact angle to the wall, this has to be taken into account. O' Brien and Hermann [87] used the equation $\left(\cos \left(\theta_{\text {bead }}\right)+\cos \left(\theta_{\text {wall }}\right)\right) / 2$ for different contact angles.
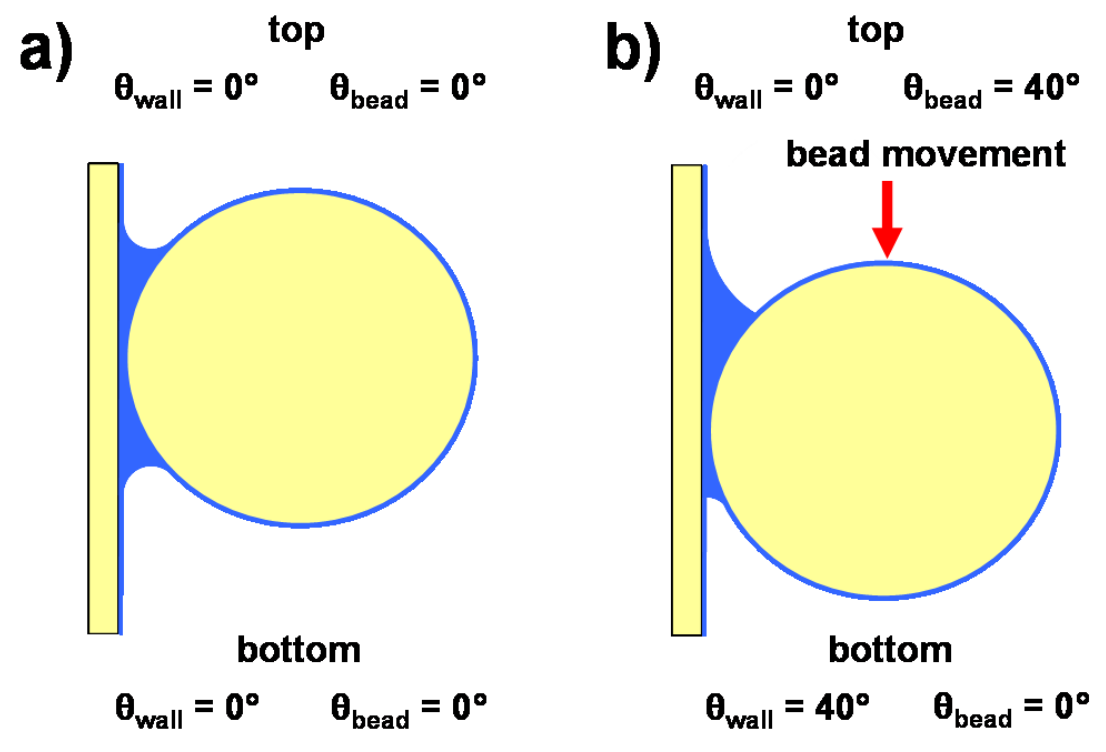

Figure 3.4: Schematic of a capillary bridge between a glass bead and a glass wall. a) Both contact angles, top (bead and wall) and bottom (bead and wall) are $0^{\circ}$. b) After movement of the bead the contact angles changes between a receding $\theta=0^{\circ}$ and an advancing angle $\theta=40^{\circ}$.

In addition, the dynamic contact angles at the critical fluidization acceleration are even more complicate. In Figure 3.4 a) a schematic of a equilibrated capillary bridge between 
a glass bead and a glass wall is shown. All contact angles between the wall/liquid and bead/liquid are zero. After moving the bead the contact angles have changed as shown in Figure $3.4 \mathrm{~b}$ ). The contact angles are in the interval between receding $\theta_{\text {rec }}=0^{\circ}$ and advancing angle $\theta_{a d v}=40^{\circ}$ of the liquid (in our case for water) on the surface of the glass beads. The top part of the wall capillary bridge has $\theta_{\text {wall }}=0^{\circ}$ and $\theta_{\text {bead }}=40^{\circ}$ and the bottom part has $\theta_{\text {wall }}=40^{\circ}$ and $\theta_{\text {bead }}=0^{\circ}$. Along the radius of the capillary bridge the contact angle changes continuously between the receding and advancing angle. In the dynamical case of the sinusoidal bead movement during vertical agitation, the contact angle changes also with time. This is by far too complicated to take it into account. We therefore simplified our system by only considering the wall contact angle $\theta_{\text {wall }}$ and the bead contact angle $\theta_{\text {bead }}$, even if small contact angles are increased and large contact angles are decreased during agitation.

The total force exerted on the granular pile by all capillary bridges at the wall can be described by the following equation:

$$
F_{\text {cap }}=4 \pi R \gamma \frac{\cos \theta_{\text {bead }}+\cos \theta_{\text {wall }}}{2} \cdot \rho_{2 D_{\text {c cub }}} O_{S}
$$

Where $\theta_{\text {bead }}$ is the contact angle of the liquid with the bead surface and $\theta_{\text {wall }}$ is the contact angle with the container surface. The surface tension of the liquid is $\gamma$. The wall surface area is $O_{S}=2 \pi r H, r$ is the container radius and $H$ is the filling height of the sample.

The $2 \mathrm{D}$ density of the capillary bridges at the wall can be approximated by that of a cubic packing $\rho_{2 D_{-} \text {cub }}=0.25 / R^{2}$, where $R$ is the radius of the bead. Experimentally we find a capillary bridge density of $0.21 \pm 0.01 / R^{2}$, which is even below the cubic packing that was used in the following calculations.

The acceleration force of the sample can be written as [81]:

$$
F_{a c c}=\left(\Gamma_{C}-\Gamma_{0}\right) \rho_{s} V g
$$

Where $\Gamma_{0}=1.2$ is the measured critical fluidization acceleration. The sample density (the mass of the grains divided by the total sample volume) is $\rho_{S}=\rho_{\text {packing }} \cdot \rho_{\text {mass }}$, where $\rho_{\text {packing }}$ is the packing density of the grains and $\rho_{\text {mass }}$ is the mass density of the grain material. The total volume of the sample is $V=\pi r^{2}$, where $H$ is the filling height of the sample.

At the critical fluidization acceleration $\Gamma_{C}$ the force due to the external agitation exceeds the force of the capillary bridges to the wall. One 'component' of the force comes from the bottom 
capillary bridges which are elongated and finally ruptured. The second 'component' comes from the capillary bridges on the side walls. The force due to all the wall capillary bridges exceeds the force due to bottom capillary bridges for our sample geometries and we assume the bottom to be negligible. The grains with capillary bridges at the side walls are sheared against the side walls. A relative motion of the bead will occur, if the agitation force of the container exceeds the maximal shear force, which is the force of the wall capillary bridges times the friction coefficient.

$$
F_{a c c} \geq \mu \cdot F_{c a p}
$$

The resulting force balance yields:

$$
\Gamma_{C}=\Gamma_{0}+\frac{2 \pi\left(\cos \theta_{\text {bead }}+\cos \theta_{\text {wall }}\right) \mu}{\rho_{S} g r} \cdot \frac{\gamma}{R}
$$

A consequence of this force balance, improved by the bead and the wall contact angle as well as the friction coefficient, is that the critical fluidization acceleration must scale with the surface tension $\gamma$ and inversely with the bead radius $R$. We tested this dependence using different bead sizes and different liquids as listed in Table 3.1 .

\begin{tabular}{|c|c|}
\hline liquid & surface tension $\gamma[\mathrm{mN} / \mathrm{m}]$ \\
\hline \hline fluorinated oil FC40 & 16 \\
ethanol & 22 \\
olive oil & 36 \\
ethylene glycol & 46 \\
diiodomethane & 51 \\
water & 73 \\
\hline
\end{tabular}

Table 3.1: Surface tension of liquids after Lide, Handbook of Chemistry, or data sheets from the manufacturer.

A typical result for the dependence of $\Gamma_{C}$ on the surface tension is displayed in Figure $3.5 \mathrm{a}$ ) for a liquid content $W=0.01$ and glass beads with $R=280 \mu \mathrm{m}$. The contact angles $\theta_{\text {bead }}$ of the used liquids and $\theta_{\text {wall }}$ are equal to zero. The error bars in Figure 3.5 correspond to the standard deviation of three or more individual measurements.

The data point for water is lower than the blue line for a linear dependence in Figure 3.5 a). A possible explanation is that the surface tension of the water had decreased to $60-65 \mathrm{mN} / \mathrm{m}$ 

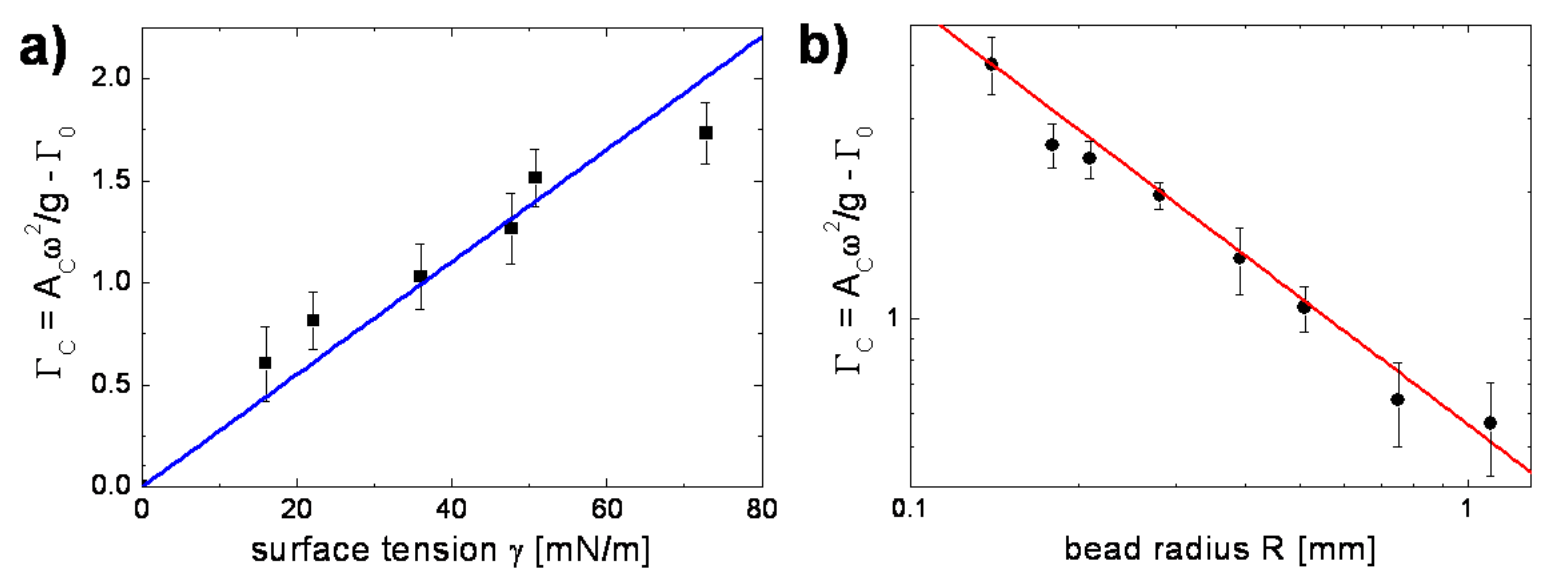

Figure 3.5: a) Critical fluidization acceleration for liquids with different surface tensions $\gamma$ at a liquid content $W=0.01$ and a bead radius $R=280 \mu \mathrm{m}$. b) The critical fluidization acceleration as a function of the bead radius $R$ at a liquid content $W=0.01$ shows a inversely bead radius $R$ dependence in a log-log plot.

due to the presence of the larger interface with the granulate and the air. If we take this into account, then the expected linear dependence on the surface tension is visible in the critical fluidization acceleration. The linear line in Figure 3.5 a) was calculated by Eq. 3.4 and a friction coefficient $\mu=0.24$ was used for the glass beads on the glass wall of the sample. A friction coefficient $\mu$ of 0.24 for glass beads on a glass wall is very likely, since a literature value is 0.4 for the dynamical friction coefficient [88]. In addition, the friction coefficient depends on the surface roughness of the material [88]. The friction coefficient $\mu=0.24$ should be constant for all bead sizes. The liquid content of the water used was $W=0.01$ and we used beads with radii ranging from $140 \mu \mathrm{m}$ to $1100 \mu \mathrm{m}$. The detection of the critical fluidization acceleration is difficult with smaller beads, where the grains movement becomes harder to detect. Moreover larger beads are not small compared to the container radius, where wall ordering effects are dominant. The measured values are shown in Figure 3.5 b). The error bars correspond to the standard deviation of four or more measurements. Again, we found the dependence predicted of Eq. 3.4 as shown by the red line, without using a fit parameter.

To quantify the influence of different bead materials we repeated the same fluidization measurements for basalt beads instead of using glass beads. The RMS roughness of the basalt beads is $8 \mathrm{~nm}$ and about one decade smaller than the glass beads used, with $60 \mathrm{~nm}$ roughness. These values were determined as described in chapter 2. We measured the typical contact 
angle of the basalt beads which is $\theta_{\text {pile }}=70^{\circ}$. This large typical contact angle influences the mechanical behavior of the bead pile. The critical fluidization acceleration for basalt beads with a bead radius of $R=280 \mu \mathrm{m}$ as a function of the liquid content (black dots) is shown in Figure 3.6 in a semi log-plot together with the results obtained with glass beads (red dots) of the same size. The error bars correspond to the standard deviation of five individual measurements. The lower plateau value of the critical fluidization acceleration of the basalt beads $\Gamma_{C}=2.8$ compared to glass beads $\Gamma_{C}=3.4$, results from the different contact angles as discussed below.

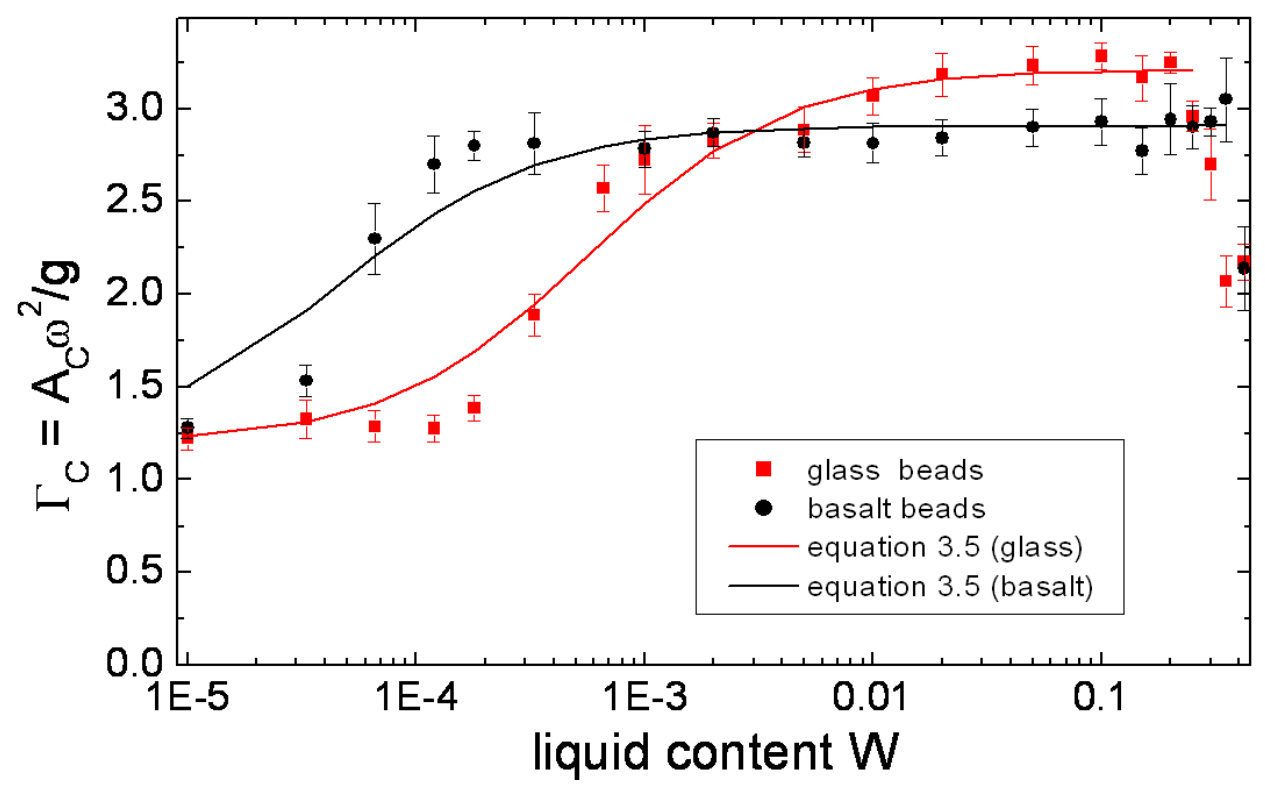

Figure 3.6: Critical fluidization acceleration as a function of the liquid content of glass beads and a contact angle $\theta_{\text {pile }}=0^{\circ}$ compared to basalt beads $R=280 \mu \mathrm{m}$ with a larger contact angle $\theta_{\text {pile }}=70^{\circ}$ between the beads.

Both curves in Figure 3.6 posses the same generic features; they increase from $\Gamma_{C}$ dry to their respective plateau values. However, $\Gamma_{C}$ for basalt beads reaches the plateau value for a lower liquid content $W_{r}$ than glass beads. This critical liquid content for the roughness regime can be characterized by an equation $W /\left(W+W_{r}\right)$ [76].

Eq. 3.4 can be modified by the liquid content of the roughness regime:

$$
\Gamma_{C}=\Gamma_{0}+\frac{2 \pi\left(\cos \theta_{\text {bead }}+\cos \theta_{\text {wall }}\right) \mu}{\rho_{S} g r} \cdot \frac{\gamma}{R} \cdot \frac{W}{W+W_{r}}
$$


The theory lines in Figure 3.6 are calculated from Eq. 3.5. In addition, the glass has a density of $2450 \mathrm{~kg} / \mathrm{m}^{3}$ and basalt has $2900 \mathrm{~kg} / \mathrm{m}^{3}$. The packing density of the basalt beads is 0.60 which is slightly larger than glass beads. We used a friction coefficient of 0.24 for glass beads and 0.30 for basalt beads on the glass walls. The liquid content of the roughness regime was determined by fitting Eq. 3.5 to the fluidization data, i.e. for basalt beads $W_{r}=(5.0 \pm 1.0) \cdot 10^{-5}$ and for the glass beads $W_{r}=(5.6 \pm 1.0) \cdot 10^{-4}$. This is the volume of liquid needed to wet the beads. It can be calculated with the measured surface roughness in chapter 2 . We assume that a liquid wetting layer on the beads has a height similar to the corresponding RMS roughness of the bead. The RMS surface roughness of basalt beads is $8 \mathrm{~nm}$ and $60 \mathrm{~nm}$ for glass beads. This calculation gives a liquid content of $W_{r_{-} \text {calc }}=(4.9 \pm 1.8) \cdot 10^{-5}$ for basalt beads. It is in agreement with the value found with the critical fluidization acceleration. The same approximation for the rougher glass beads results in a liquid content of $W_{r_{-} \text {calc }}=(3.7 \pm 1.0) \cdot 10^{-4}$, which is again in agreement with the critical fluidization acceleration. This means that the smoother the surface of the used beads, the smaller the liquid content $W_{r}$ of the roughness regime where the stiffness of the wet granulate is increased.

At large liquid content $W>0.2$ the measurements are difficult to perform, since the liquid layers at the wall include air bubbles whose motion can be misinterpreted as movement of the grains. In the completely submerged case, where all air in the pile is replaced by liquid, both bead materials have a similar critical fluidization acceleration $\Gamma_{c}$ of approximately 2.1.

To evaluate the influence of the grain shape we varied not only the bead material, but repeated the same measurements for sand grains. The sand (received from a glacial deposit near Marzahne, Brandenburg, Germany) consists mainly of $\mathrm{SiO}_{2}$ and is comparable to glass beads, but with irregular shapes and with a RMS surface roughnesses of approximately $0.1 \mu \mathrm{m}$ to 0.5 $\mu \mathrm{m}$. The sand size was sieved to $355-500 \mu \mathrm{m}$ in order to be comparable to glass beads with the radii in a range of $450-500 \mu \mathrm{m}$. The critical fluidization acceleration as a function of the liquid content is shown in Figure 3.7 [62]. Sand grains have a $30 \%$ higher plateau value than glass beads with the same mean diameter. The error bars correspond to the standard deviation of three or more individual measurements.

The vertical critical fluidization acceleration is a fast and simple measurement technique which enabled us to compare many different parameters such as grain sizes, grain materials, surface tensions of the liquid, and liquid contents. However, many parameters influence the measurements such as the history of the sample, the bead surface, the wall properties, the dy- 


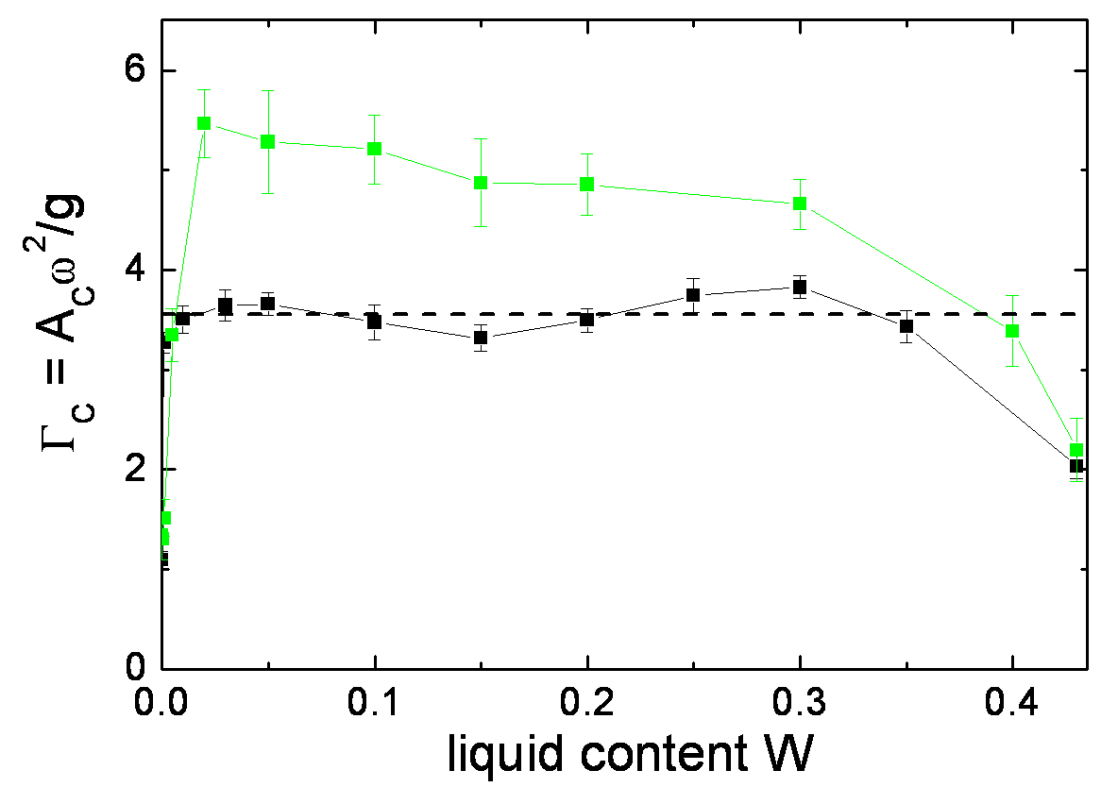

Figure 3.7: The critical fluidization acceleration as a function of the liquid content for sand grains (green squares, size 355 - $500 \mu \mathrm{m}$ ) [62] and glass beads (black squares, size 450 $500 \mu \mathrm{m})$. The dashed line denotes the plateau of the critical fluidization acceleration calculated by Eq. 3.4 .

namic contact angles, the grains in a sample losing contact with the wall, and the drainage of the liquid at larger liquid contents results in difficulties when comparing measurements taken for different parameters. 


\subsection{Shear stiffness measurements}

The shear stiffness of wet grains can be measured using a standard rheometer [89]. A standard rheometer consist of two cylinders, of which the inner cylinder is able to rotate in the outer one. The grains are placed between the cylinders and are sheared by oscillation of the inner cylinder. The difficulties in using this device on wet granulates is the formation of shear cracks. In the worst case the grains are not sheared between the two walls but rather the granulate completely loses contact with one of the walls. Thus, without an additional technique to detect these cracks, the results from a standard rheometer are very difficult to interpret. Furthermore, in a standard rheometer only small beads can be used, because the strength of a capillary bridge scales inversely with the bead radius $R$. With larger grains the measurement signal is very weak and the signal to noise ratio is too small to provide quantitative measurements [89].

For the shear measurements presented here we used a shear cell to measure the stiffness of wet granulates. We measured the stiffness of the granulate at a constant shear velocity. This parameter we call 'shear stiffness'. The shear stiffness is a special case of the yield stress, which depends on the definition and is experimentally difficult to estimate [90]. We measured the shear stiffness of the granulate as a function of the liquid content in order to compare the results with the critical fluidization acceleration measurements. In addition, the shear stiffness as function of the absolute pressure was measured in order to obtain information about the friction between the grains. The shear stiffness measurements were performed by Michael Sipahi [91].

\subsubsection{Measurement technique}

Due to the limitations mentioned above in using a standard rheometer we used a shear cell where the grains are sheared with a parabolic profile between two membranes [58, 81]. A schematic of the shear cell is shown in Figure 3.8 at zero and maximal shear amplitude. The cell is built out of a plastic ring with a $3 \mathrm{~cm}$ diameter and has a thickness of $1 \mathrm{~cm}$. The ring is closed at both ends by rubber membranes of about $0.3 \mathrm{~mm}$ thickness. The wet grains are placed between the membranes. The two membranes, sealing liquid reservoirs, are connected with Teflon ${ }^{\circledR}$ tubing (PTFE, polytetrafluoroethylene) with $1 \mathrm{~mm}$ inner diameters, 

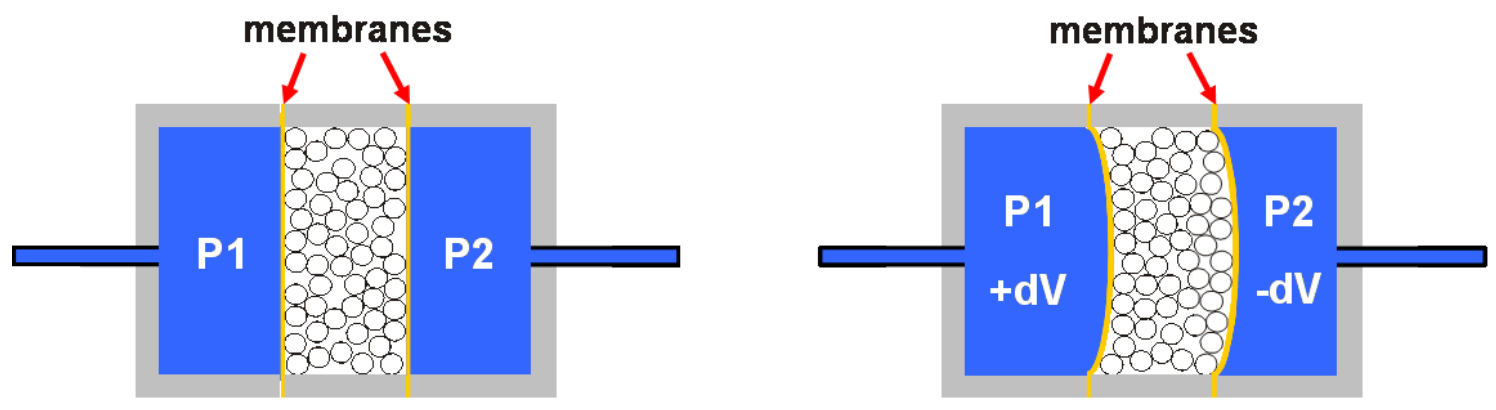

Figure 3.8: Schematic of the shear cell. The left shear amplitude is zero and at the right the shear amplitude is at its maximal value.

to syringe pumps. Two pressure sensors, P1 and P2, measure the absolute pressure of both water reservoirs. A third pressure sensor, P3, measures the differential pressure between the two reservoirs and is used as a control system to the differential pressure (P2 - P1).

We used the pressure sensors 24PCC (with a pressure range of -100 $\mathrm{kPa}$ to $100 \mathrm{kPa}$ ) purchased from RS Components $\mathrm{GmbH}$. In order to get an absolute signal these sensors have to be calibrated. The voltage signals of the three pressure sensors were measured by a National Instruments measurement card NI DAQPAD-6015 and recorded by a PC using LabVIEW 8.5.1. To test the linearity of the pressure sensors within the required range, we plotted the results of known pressure signals in Figure 3.9 [91]. The known pressure (blue line) was generated by applying a mass onto a surface of a piston. Both sensors, P1 (black dots) and P2 (red dots), show a linear dependence on the applied pressure. The measured pressure values deviate less than $5 \%$ from the applied pressure calculated from the mass and the surface area of the piston. We calibrated our sensors accordingly and found the output to be stable over long periods of time.

To shear the granulate we increased the volume in reservoir 1, decreased the volume in reservoir 2, and measured the resulting differential pressure. The liquid volume was varied using two precision syringe pumps. The pumps were driven with a periodic saw-tooth signal with the same absolute value of the slope on both sides. The value of the shear amplitude used is important, since a shear amplitude that is to small will not shear the grains irreversibly. Therefore the shear amplitude at the center of the shear cell was measured for various shear volumes as shown in Figure 3.10 [91]. The error bar corresponds to the precision of the amplitude measurement. We found that the shear amplitude $A$ (black dots) scales linearly (orange line) with the pumped volume $V$. In our experiments we used an amplitude of $0.8 \mathrm{~mm}$, visu- 


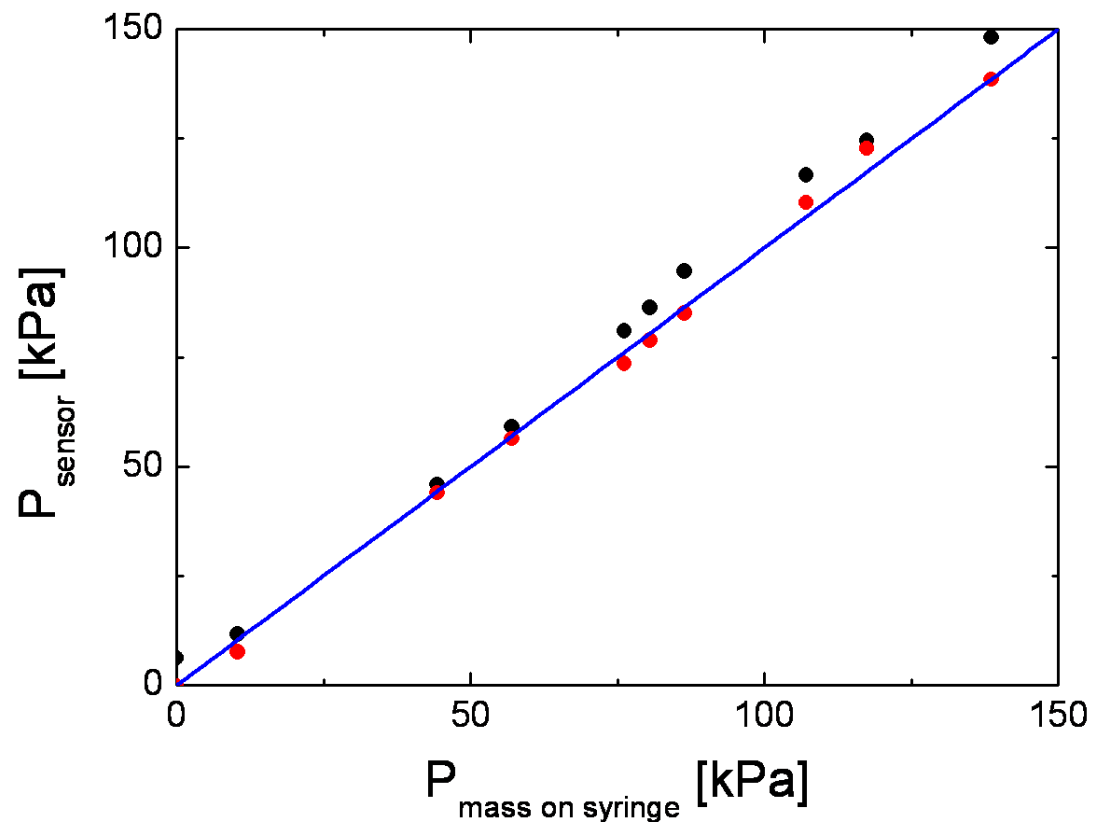

Figure 3.9: Linearity test of an applied pressure (blue line) and the measured pressures of the used pressure sensors P1 (back dots) and P2 (red dots) [91].

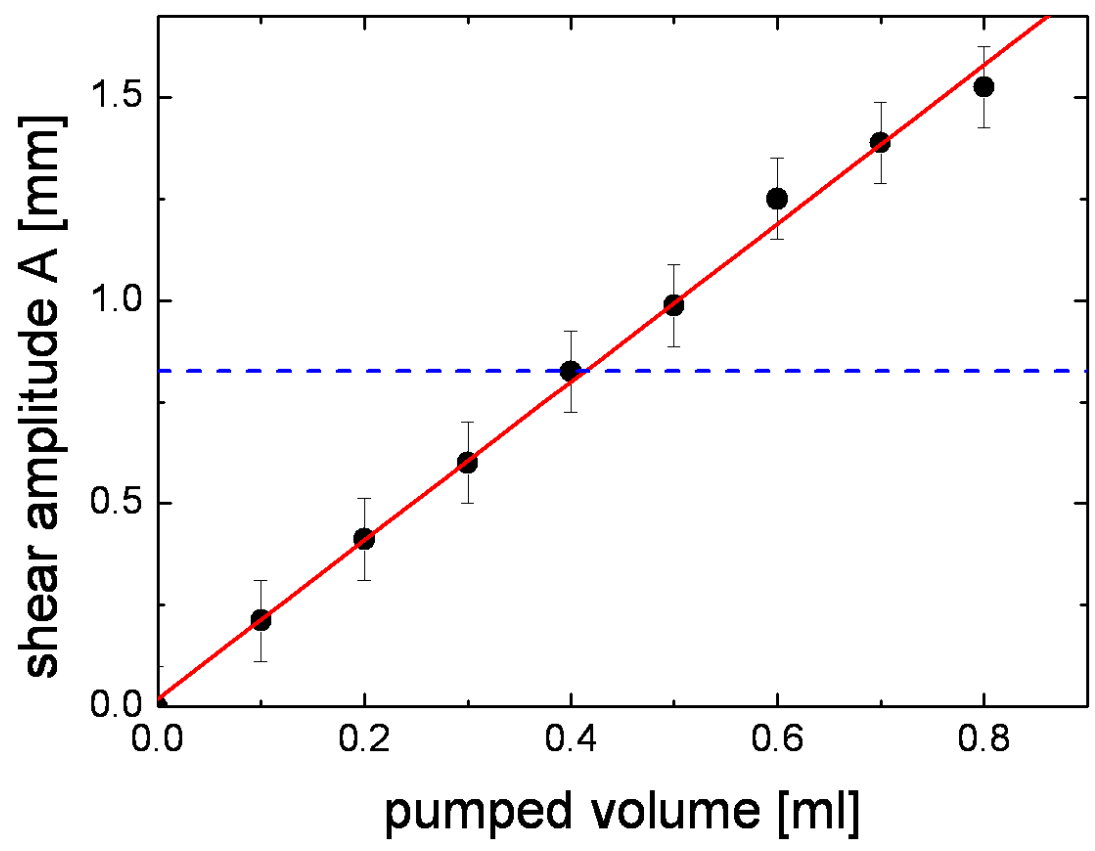

Figure 3.10: Shear amplitude (black dots) as a function of the pumped volume. The orange line shows a linear dependence and the dashed blue line shows the mostly used shear amplitude [91]. 
alized as a dashed blue line in Figure 3.10. The shear amplitude is much larger than the radius of the beads with $68 \mu \mathrm{m}$, so that many beads have to rearrange their positions with respect to the surrounding beads. Before a measurement was started the wet grains were sheared for more than ten cycles and a defined absolute pressure of $4 \mathrm{kPa}$ was applied. This pre-shearing was performed in order to obtain reproducible results.
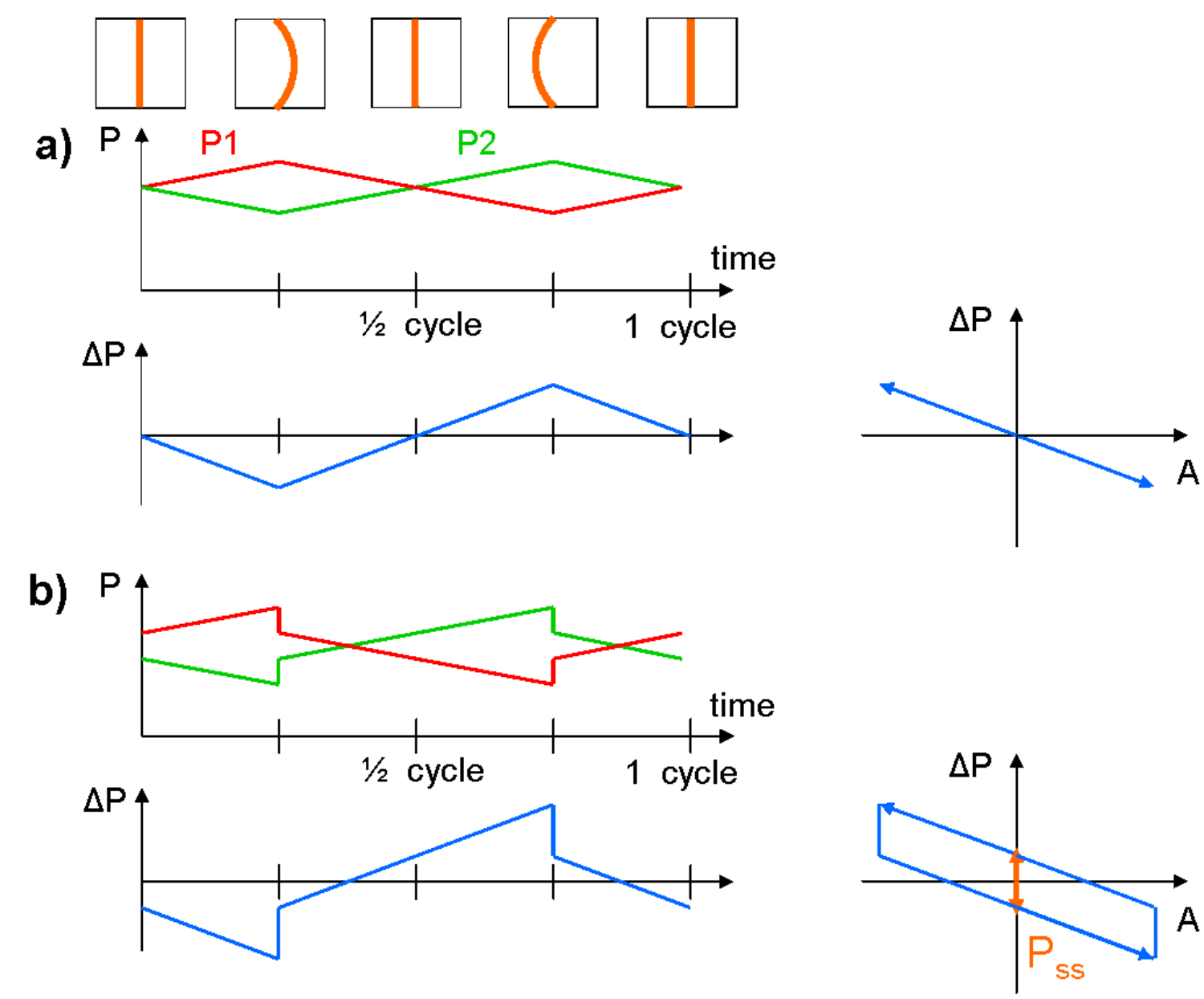

Figure 3.11: Schematic pressure signals during a shear cycle. The absolute pressure of the two sensors on left and right side of the shear cell (red and green lines), the differential pressure signal (blue line). The right graph shows the resulting hysteresis loops. Figure a) for an elastic membrane and $b$ ) for a granulate.

We will now use a schematic shear cell to explain the pressure signals, this is shown in Figure 3.11. On top the position of the membranes is sketched. The shear cycle is explained from left to right following the time axis of Figure 3.11 . We start with straight membranes as 
indicated on top. a) Illustrates the signals for an elastic membrane without granulate and b) with granulate filled into the shear cell. For shearing the granulate the volume in one reservoir was decreased and increased in the other reservoir by the same amount. This volume corresponds to a certain shear amplitude $A$. The upper graph of Figure 3.11 a) shows the time dependence of the absolute pressure in both cells. The red line shows a linear increase, if the volume is increased while the pressure in the other reservoir, corresponding to the green line, decreases linearly as shown due primarily to the elasticity of the membrane as well as the elasticity of the tubing system. After one fourth of a shear cycle the shear direction is reversed and accordingly the slope of the pressure signals change signs. At three fourth of the cycle the shear direction has reversed to the original direction. The lower left graph in Figure 3.11 a) shows the resulting differential pressure of the reservoirs, P2 - P1 (or P3 respectively). The right graph shows the differential pressure plotted against the shear amplitude. In an elastic system with only rubber membranes in the shear cell works reversibly and the elastic energy of the membranes lost by pushing will be regained by pulling. Only a small opening of the 'hysteresis' loop will be visible due to the small inelasticity of the membranes.

In the case where a granulate is sheared our expected results are shown in Figure $3.11 \mathrm{~b}$ ). Here steps are visible in the pressure signals. The inelastic granulate needs more force at the side pushing the granulate than at the other side moving away from the granulate. This is visible as jumps in the pressure signals. In the right graph of Figure 3.11 b) the differential pressure signal is plotted against the shear amplitude and an opening is observed. The opening height of this 'hysteresis' loop will be used as measurement signal and we label this pressure value as the shear stiffness $P_{s s}$. We want to compare the shear stiffness with the critical fluidization acceleration.

\subsubsection{Experimental results}

Having discussed a shear cycle of a virtual shear cell, we now show typical signals from a 'real' shear experiment in Figure 3.12 a). We see in a) that the absolute pressure is not constant during a shear cycle. The variance of the absolute pressure can be much larger than the mean value of the absolute pressure. The value for the absolute pressure was obtained by averaging the mean pressure value over one full shear cycle. Figure 3.12 b) shows the 

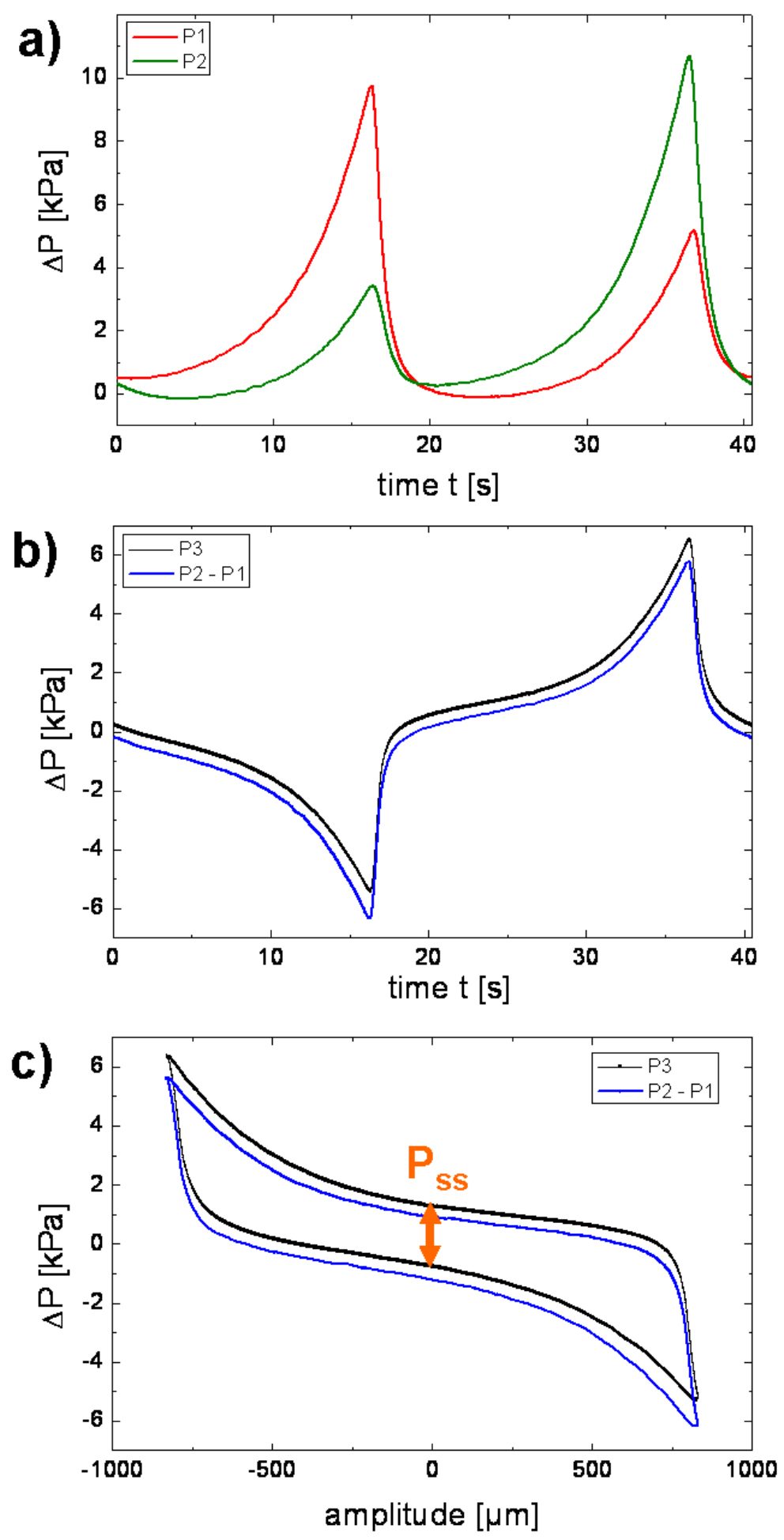

Figure 3.12: a) Absolute pressure in the two liquid reservoirs during one shear cycle. b) Differential pressure signal measured directly by the sensor P3 (black curve) and calculated from the two absolute pressure sensors (blue curve). c) Hysteresis loop from one shear cycle for both differential pressure signals. 
differential pressure from the sensor $\mathrm{P} 3$ (black) and the difference signal $\Delta P=P 1-P 2$ (blue). We shifted the curves by $0.2 \mathrm{kPa}$ from zero pressure in order to compare the curves more easily. We show an example of the differential pressure signal for one shear cycle as a function of the shear amplitude in Figure $3.12 \mathrm{c}$ ), where a hysteresis loop is visible. The shear stiffness $P_{s s}$ of the granulate is the opening of the hysteresis loop of the differential pressure plotted as a function of the shear amplitude. If we completely submerge the shear cell in water the differential pressure is close to zero as was shown in Figure 3.11 a). Only the pressure difference due to the stiffness of the membrane and the tubing is visible in the hysteresis loop. We measured $P_{s s}$ as the opening of the hysteresis loop by estimating the central area of the hysteresis loop to obtain an averaged value.

The pressure signals in Figure 3.12 show differences compared with the virtual shear cell shown in Figure 3.11, especially double peaks. This is a specific feature of the dilatancy of wet granulates and will not be discussed in detail, since it is currently being investigated by Sipahi in his thesis [91]. We observed that the packing density of our grains changes slightly during a shear cycle and the estimated change was on the order of $\Delta \rho=0.005$. This change results in a large pressure peak, since our tubing system is very stiff and any change in the sample volume will have large effects in the pressure signals, especially at the point where the shear direction is changed. On the other hand the differential pressure signals measured at the middle of the hysteresis loop are not affected by the dilatancy.

The shear stiffness $P_{s s}$ as a function of the shear amplitude for a wet granulate (black squares) with a liquid content $W=0.05$ is shown in Figure 3.13 [91], in comparison with a dry granulate (red dots). The error corresponds to the standard deviation of more than five shear cycles. The dry granulate has a small and nearly constant $P_{s s}$ of $0.8 \mathrm{kPa}$. A clear offset from the wet shear stiffness $P_{s s}$ to the dry shear stiffness is visible. The $P_{s s}$ of the wet granulate scales linearly with the shear amplitude. To increase the value of the shear stiffness a large shear amplitude is useful. Due to the design and working principle of the shear cell the maximal shear amplitude is limited to about $2 \mathrm{~mm}$. At large amplitudes the shear profile deviates from a parabolic shape. If not stated otherwise we used a shear amplitude of $0.8 \mathrm{~mm}$ which corresponds to more than ten bead radii.

To explore the influence of the absolute pressure on the shear stiffness we varied the absolute pressure $P$ from $0 \mathrm{kPa}$ to $30 \mathrm{kPa}$ as illustrated in Figure 3.14 [91]. The data for dry granulate is denoted by black squares and each data point shows the shear stiffness in one 


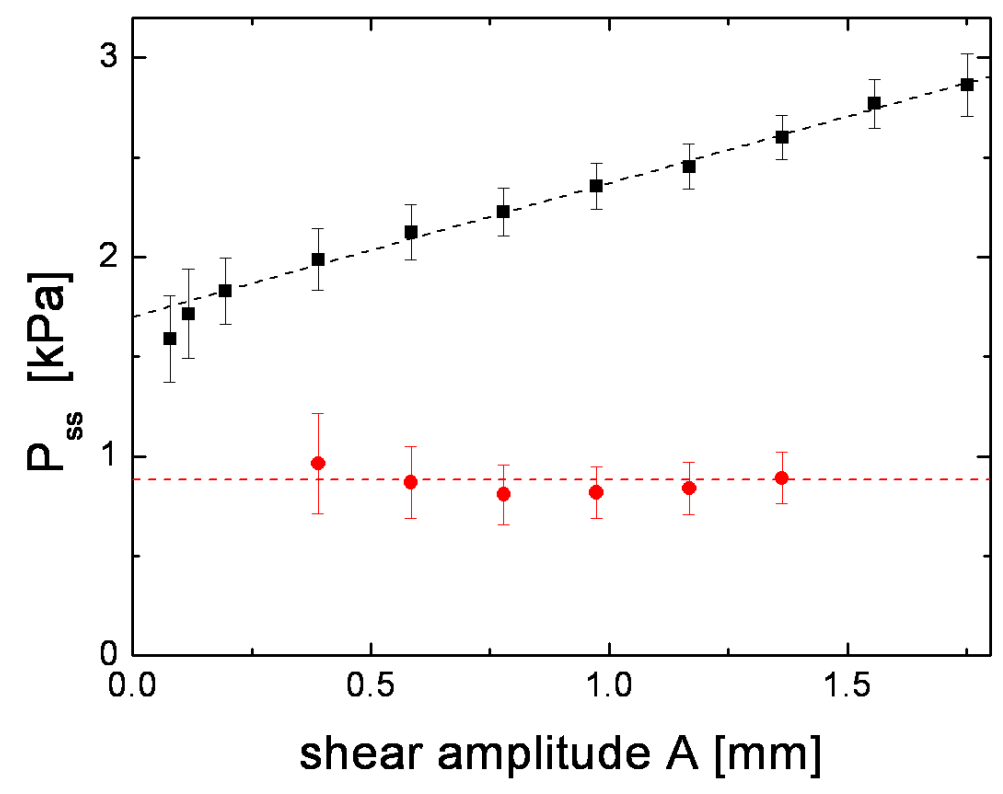

Figure 3.13: Differential pressure as a function of the shear amplitude for a dry (red dots) and a wet (black squares) granulate with a liquid content $W=0.05$ and a bead radius $R=68 \mu \mathrm{m}$, where the absolute pressure was $4 \mathrm{kPa}[\overline{91}]$.

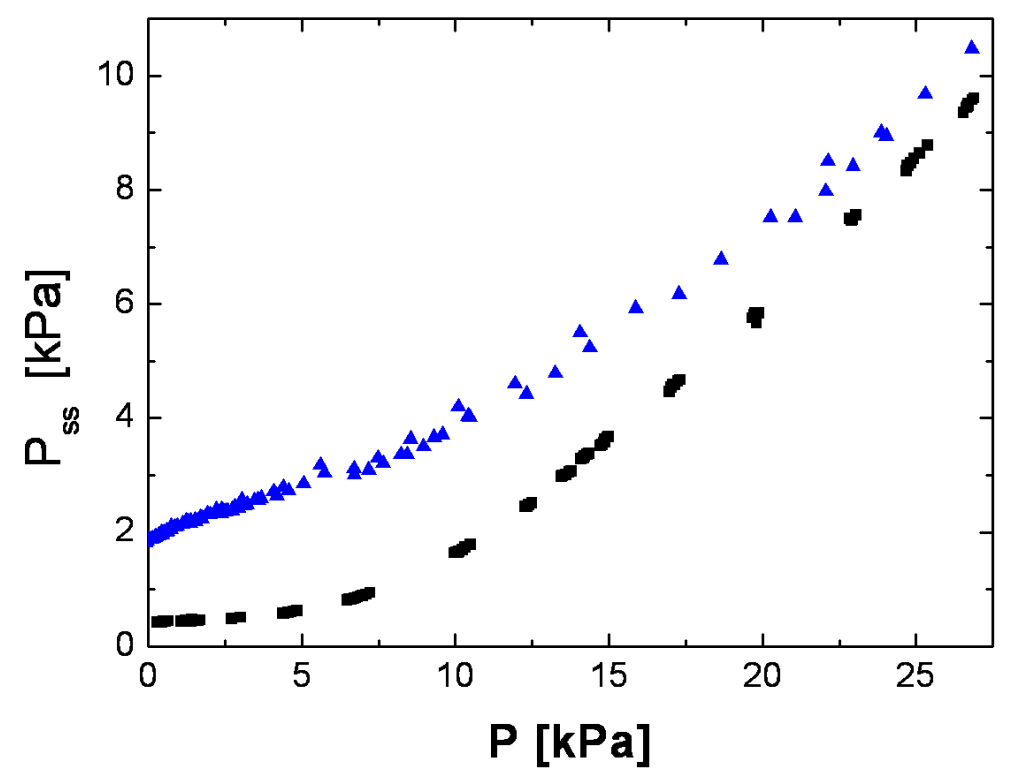

Figure 3.14: Typical results of the shear stiffness $P_{s s}$ as a function of the absolute pressure for a dry (blue triangles) and a wet granulate (black squares) [91]. The granulate consists of glass beads with a radius $R=68 \mu \mathrm{m}$ and a liquid content $W=0.05$ of water in the wet case. 
shear cycle. The measurement for a dry granulate starts at an absolute pressure $P=0 \mathrm{kPa}$ at a shear stiffness of $0.4 \mathrm{kPa}$. The stiffness of the membrane and tubing were not subtracted from these signals, because these effects were below $0.2 \mathrm{kPa}$. With increasing absolute pressure the shear stiffness $P_{s s}$ increased slowly, and at an absolute pressure $P>10 \mathrm{kPa}$ a linear increase of $P_{s s}$ was observed.

The shear stiffness of the wet granulate, the blue triangles, has an offset of $2.0 \mathrm{kPa}$ from the dry sample at $P=0 \mathrm{kPa}$. The wet shear stiffness also increases again with the applied pressure. For absolute pressures $P>15 \mathrm{kPa}$ a linear increase of $P_{s s}$ is observed with a similar slope to the one observed for a dry granulate.

The increase of the stiffness of the wet granulate is due to the capillary forces of the liquid morphologies. The linear increase of the shear stiffness is a result of friction between the grains which is proportional to the absolute pressure. At large absolute pressures friction effects dominate over the effects of the capillary forces of liquid morphologies.

The liquid dependence of the shear stiffness was measured at an absolute pressure of $4 \mathrm{kPa}$ which is large enough to be sure that the membranes are in contact with the grains and low enough, so that the friction between grains is not the dominant part of the shear stiffness.

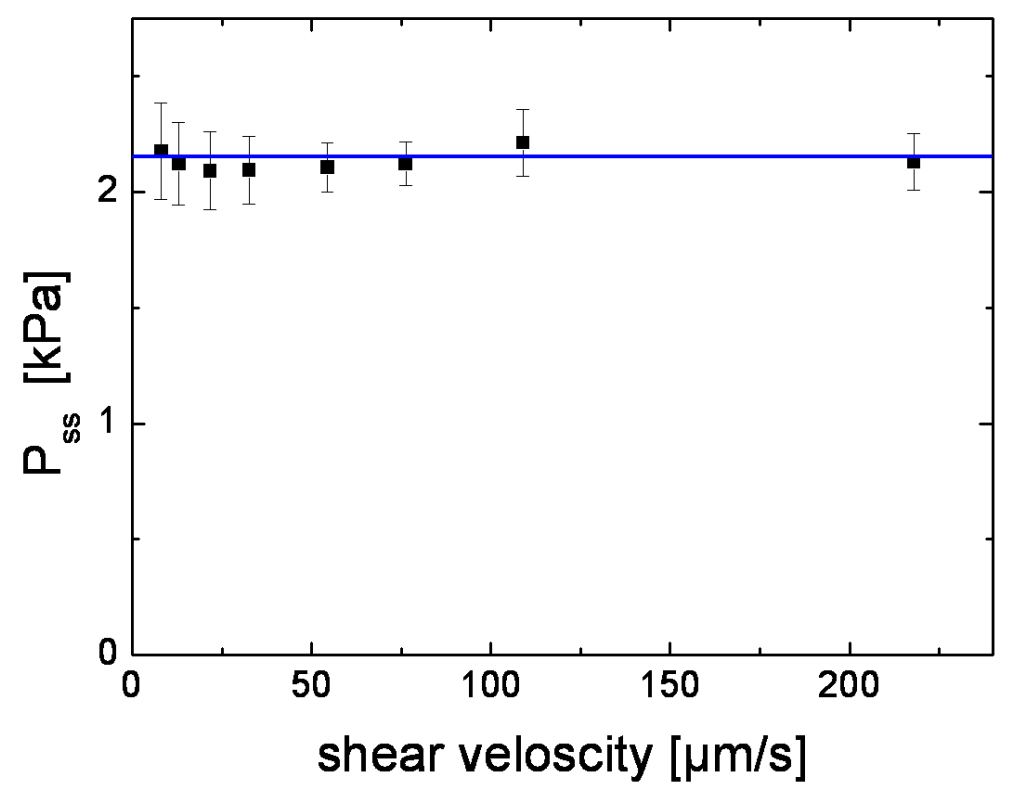

Figure 3.15: Shear stiffness $P_{s s}$ of wet granulate as a function of the shear velocity [91]. Bead radius $R=68 \mu \mathrm{m}$ and a liquid content $W=0.05$ of water and the absolute pressure was $4 \mathrm{kPa}$.

Figure 3.15][91] shows the shear stiffness $P_{s s}$ as a function of the shear velocity. The error corresponds to the standard deviation of more than five shear cycles with increased and de- 
creased shear velocities. We observed no velocity dependence in the range of 10 to $200 \mu \mathrm{m} / \mathrm{s}$, even if we systematically increased or decreased the shear velocities. At even larger shear speeds we saw an increase in the shear stiffness due to the fast flow speed of water in the tubing. We observed that slow packing rearrangements influence the shear stiffness by $10 \%$, especially if the granulate had only five presheared cycles at the preshearing step. In addition, Geromichalos [76] observed a weak dependence on the shear speed. He expected that reorganization of liquid in the sample decreases the shear stiffness. We observed only an influence of the shear stiffness by the changed packing density of the beads during many shear cycles and we can neglect the influences of the liquid on the shear velocity in our measurements.

For reproducible shear measurements of the liquid dependence a constant shear amplitude, a constant absolute pressure (mean over one cycle), and a bead radius of $50 \mu \mathrm{m}$ to $100 \mu \mathrm{m}$, must be used for our shear cell. Larger bead radii result in a smaller measurement signal, and for radii smaller than $50 \mu \mathrm{m}$, deviate the shear profile of the membranes from a parabolic shape due to cracks in the granulate.

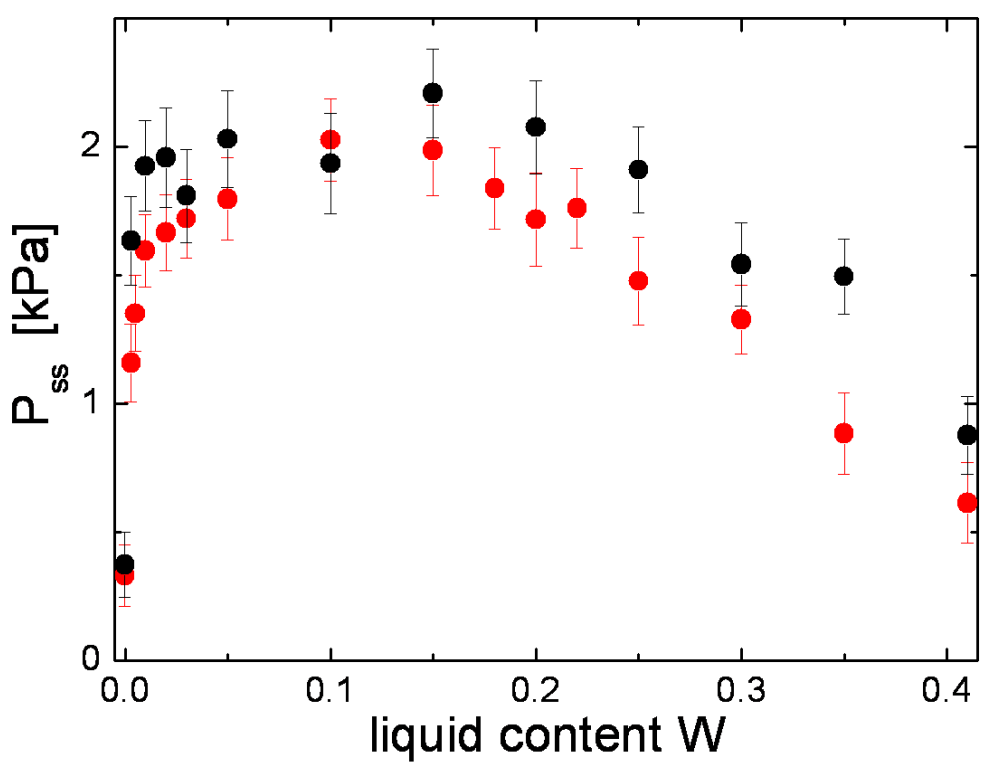

Figure 3.16: The dependence of the shear stiffness on the liquid content $W$ for glass beads (red dots) with a contact angle $\theta_{\text {pile }}=0^{\circ}$ and basalt beads (black dots) $\theta_{\text {pile }}=70^{\circ}$ [91]. Both bead types have a radius of $R=68 \mu \mathrm{m}$.

The shear stiffness $P_{s s}$ of our granulates was measured from the dry to the completely submerged case and the result is shown in Figure 3.16 [91]. The error corresponds to the standard 
deviation of more than five measurements. The red data points display the results obtained with glass beads with a radius $R=68 \mu \mathrm{m}$ and a contact angle $\theta_{\text {pile }}=0^{\circ}$. For increasing liquid content the shear stiffness $P_{s s}$ increases rapidly from $P_{s s}=0.4 \mathrm{kPa}$ at $W=0$. From $W=0.02$ the shear stiffness is further increased by about $20 \%$ to a maximum which is reached at $W=0.1-0.15$ at $P_{s s}=2.0 \mathrm{kPa}$. Further increase of the liquid content decreases the shear stiffness approximately linearly and at $W=0.41$ reaches the completely submerged state a shear stiffness of $P_{s s}=0.7 \mathrm{kPa}$.

The black data points show the shear stiffness for the basalt beads of the same size as glass beads and a contact angle $\theta_{\text {pile }}=70^{\circ}$. The dependence of the shear stiffness on the liquid content is similar to the glass beads. There is a faster increase at small liquid contents of the shear stiffness and nearly all of the other data points (black dots) in Figure 3.16 [91] have a $0.4 \mathrm{kPa}$ larger shear stiffness than a sample of glass beads.

The liquid forms complete capillary bridges between the smooth basalt beads at lower liquid contents than for the rougher glass beads, as was also observed with the critical fluidization acceleration measurements. The liquid dependence of the shear stiffness measured with the shear cell in Figure 3.16 [91] shows the same universal behavior: a low value in the dry sample and a fast increase to a plateau as was measured with vertical fluidization of glass and basalt beads in Figure 3.6 or sand in Figure 3.7. The main difference is that the shear stiffness of basalt beads is larger than it was with glass beads.

Why is the shear stiffness of basalt beads $\theta_{\text {pile }}=70^{\circ}$ at nearly all liquid contents larger than for glass beads $\theta_{\text {pile }}=0^{\circ}$ ? We expected only $1 / 3$ for the basalt beads. We found three components which increase the shear stiffness of basalt beads. One part is the increased packing density $\rho_{\text {packing }}=0.61$ of the basalt beads that makes rearrangements of the beads more difficult and results in an offset of the shear stiffness. This contribution is visible in the dry and submerged case where no capillary forces are present in the bead pile. The increased packing density increases the coordination number [92] and also the number of capillary bridges on a bead as shown in chapter 4 . Each effect increases the shear stiffness by about $10 \%$. This account for $2 / 5$ of the observed shear stiffness, which is insufficient to account for our observation.

The main contribution to the shear stiffness of basalt beads comes from stretched capillary bridges. During a shear cycle the beads are sheared. The packing density of the granulate in the shear cell increases and decreases during a shear cycle [91] as can be seen in the second pressure peak in Figure 3.12 a). When the separation distance $S$ between beads increases, 

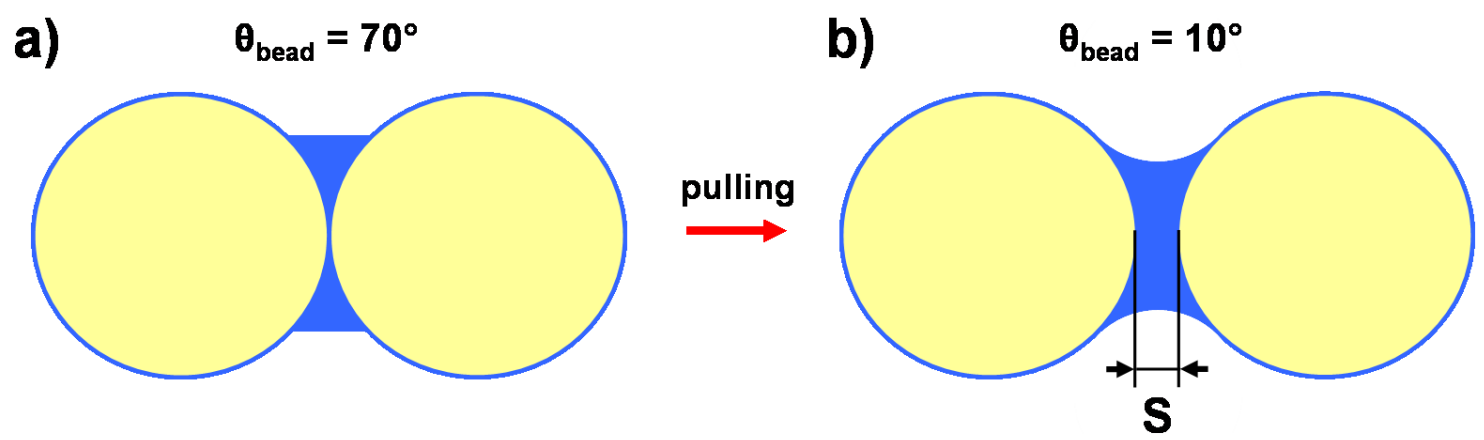

Figure 3.17: a) Schematic of a capillary bridge between basalt beads with a contact angle $\theta_{\text {bead }}=70^{\circ}$. b) The same capillary bridge after pulling the beads apart by a separation distance $S$. The large contact angle has decreased to the receding angle of $10^{\circ}$.

the contact angle decreases as shown schematically in Figure 3.17. The contact angle of the pulled capillary bridges decreases to the receding angle. The resulting capillary force of the capillary bridges increases due to the decreased contact angle $\theta_{\text {rec }}=10^{\circ}$ and decreases due to the increased separation distance as shown in chapter 1 by Willet et al. [34]. To generate an order of magnitude estimate for the force, we considered a cylindrical capillary bridge with an half opening angle $\beta=20^{\circ}$ and a contact angle $\theta_{\text {bead }}=70^{\circ}$ as illustrated in Figure 3.17 a). The capillary force is decreased by the cosine of the contact angle $\cos \left(\theta_{\text {bead }}=70^{\circ}\right)$ and has a value of 0.34 compared to 1 for $\theta=0^{\circ}$. If we stretch the capillary bridge by the separation distance normalized by the bead radius, $S^{*}=0.03$, the contact angle decreases to the receding angle $\theta_{\text {rec }}=10^{\circ}$ as illustrated in Figure $3.17 \mathrm{~b}$ ). The capillary force is now only decreased by the separation distance $S$ and has 0.84 of the maximal capillary force. The capillary force is more than doubled for a capillary bridge between beads with a separation distance, when compared with a capillary bridge between beads in contact. In the shear cell during a shear cycle some beads increase their separation distance $S$, some beads decrease $S$, and most beads are still in contact. The contact angle increases to the advancing angle $\theta_{a d v}=85^{\circ}$ and results in an almost zero capillary force for beads which have a capillary bridge between them and decrease their separation distance. If we sum over all of the capillary bridges, an increase of the shear stiffness will be obtained, but how large it will be is difficult to estimate. In addition, this process only occurs in systems with large contact angles and small separation distances. This effect discussed above of the stretched capillary bridges on the shear stiffness can be better characterized in a system where all capillary bridges are pulled. This has been done using a tensile strength measurement and is discussed in the next section. 


\subsection{Tensile strength measurements}

The general idea in measuring the tensile strength is to stretch a wet granulate pile until it ruptures. The maximal force that can be applied before rupture occurs is a measure of the tensile strength. Measurements were previously done by Pierrat et al. [73] and Richefeu et al. [74]. They filled wet grains into a cell containing two halves which can be split. One side was pulled and at the connection area between the two halves the pile broke. The drawback of this technique is that the position of the crack is predetermined and influences the tensile strength. Instead we implemented an improved technique where the crack position is not predefined. In the following section we will explain the measurement principle used in our tensile strength setup and discuss the results.

\subsubsection{Measurement technique}
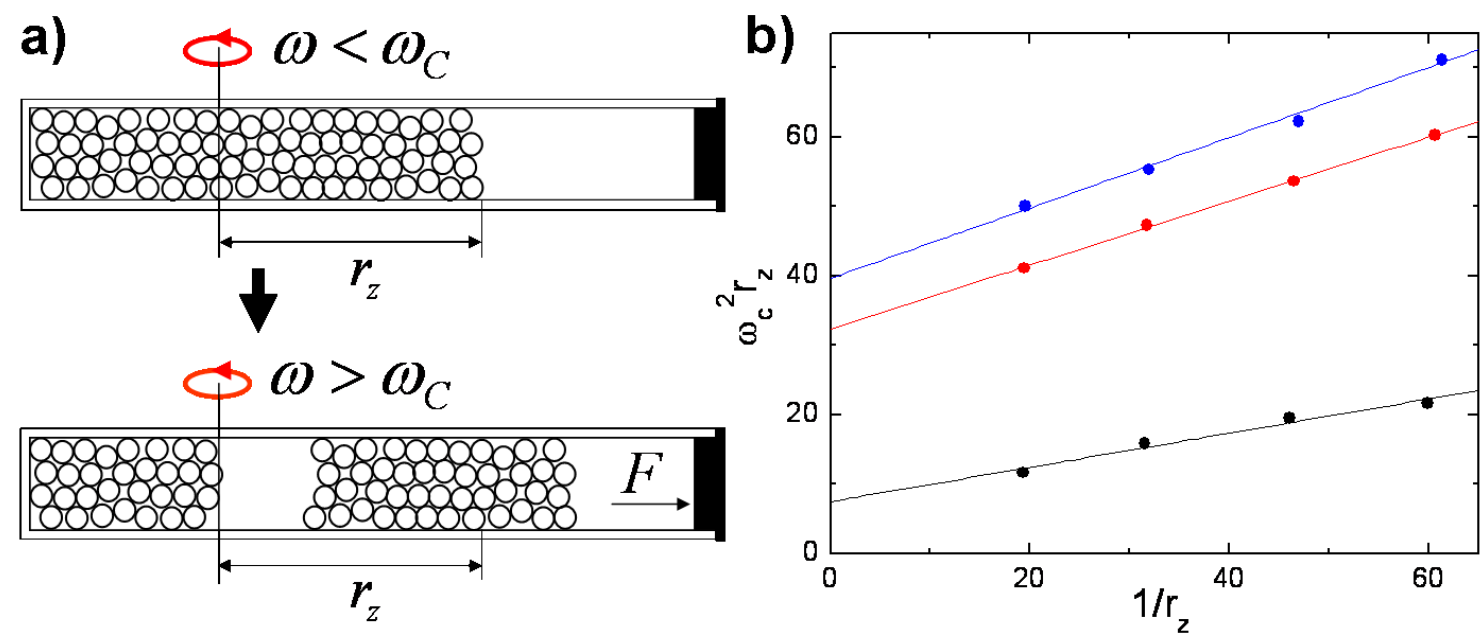

Figure 3.18: a) Schematic of the centrifuge before and after a pile rupture [79]. b) Typical rupture plot for a wet granulate as a function of different arm lengths. The beads radius is $R=141 \mu \mathrm{m}$ and liquid contents of $W=0.001$ (black dots), $W=0.01$ (red dots), and $W=0.05$ (blue dots).

As sample container we used a $20 \mathrm{ml}$ plastic syringe (polypropylene) with an inner diameter of $20 \mathrm{~mm}$ and a length of $70 \mathrm{~mm}$. After adding a liquid content $W$, the syringe was shaken, mixed with a lab spoon and shaken again until a homogenously wetted granulate was 
obtained. To generate a reproducible packing density the sample was tapped one time on the bottom of the syringe and then compressed from the top by applying a fixed force of $50 \mathrm{~N}$. The final packing for glass beads is $\rho_{\text {packing }} 0.59 \pm 0.01$. The samples were mounted on a rotating stage. The arm length $r_{Z}$ is the distance of the free end of the wet granulate to the symmetry axis of the rotating stage as shown in Figure 3.18 a) [79] top. The angular velocity $\omega$ was slowly increased until the granular pile in the sample container ruptured. This rupture is shown schematically in Figure 3.18 a) [79]. The wet granular pile in the plastic syringe always ruptures close to the symmetry axis. The arm length $r_{Z}$ of the granulate was varied and the angular velocity $\omega_{c}$ at rupture was recorded and used to calculate the tensile strength and the friction coefficient.

The tensile strength can be calculated from the force balance of the centrifugal force and the tensile force plus a friction force.

$$
F_{\text {centrifugal }}=F_{\text {tensile }}+F_{\text {friction }}
$$

The centrifugal force of the pile inside the centrifuge can be calculated by the integral:

$$
F_{\text {centrifugal }}=\int_{0}^{r_{Z}} \rho_{S} A \omega_{c}^{2} x d x=\rho_{S} A \omega_{c}^{2} r_{Z}^{2} / 2
$$

where $\rho_{S}=\rho_{\text {packing }} \cdot \rho_{\text {mass }}$ is the mass density of the sample, $\rho_{\text {packing }}$ is the packing density of the granulate and $\rho_{\text {mass }}$ is the mass density of the bead material. $A$ is the cross sectional area of the syringe and $r_{Z}$ is the arm length from the rotating axis to the free end of the pile as shown in Figure 3.18 a) and $\omega_{c}$ is the angular velocity where the pile ruptured.

The tensile force is defined as the tensile strength $\sigma$ multiplied by the cross sectional area $A$.

$$
F_{\text {tensile }}=\sigma \cdot A
$$

The friction force can be calculated from the wall capillary bridge force, the capillary bridge density and the surface area of the capillary bridges $O_{S}=2 r \pi r_{Z}$, where $r$ is the radius of the tube:

$$
F_{\text {friction }}=\mu F_{\text {cab }} \rho_{2 D_{-} \text {cub }} O_{S},
$$

$\mu$ is the friction coefficient between the beads and the container walls. The wall capillary 
bridge force is $F_{c a p}=4 \pi \gamma R \cos (\theta)$ as shown in the section critical fluidization acceleration measurements. The $2 \mathrm{D}$ capillary bridge density at the container walls for a cubic packing is $\rho_{2 D \_c u b}=0.25 \cdot 1 / R^{2}$ and experimentally a capillary bridge density of $0.21 \pm 0.01 / R^{2}$ was found, as explained previously in the section critical fluidization acceleration.

There is no need to take gravity into account, because gravity increases the attractive force of the lower capillary bridges in the tube and decreases the attractive forces of the upper capillary bridges equally so that the effects cancel out.

In order to extract the tensile strength $\sigma$ and the friction coefficient $\mu$ in a straightforward manner we obtained data for different arm lengths as shown in Figure $3.18 \mathrm{~b}$ ). We determined the tensile strength by plotting $1 / r_{z}$ as the independent variable and $\omega_{c}^{2} r_{Z}$ as the dependent variable. The tensile strength was taken as the slope $m$ of a linear fit to that data.

Rewriting the force balance using the terms mentioned above,

$$
\omega_{c}^{2} r_{Z}=\frac{2 \sigma}{\rho_{S} r_{Z}}+\frac{4 \mu \pi \gamma \cos \theta}{\rho_{S} r R}
$$

The tensile strength is given by $\sigma=m \cdot \rho_{S} / 2$ and the intercept of the Y-axis gives the wall friction coefficient $\mu=\frac{Y_{\text {intercept }} \rho_{S} r R}{4 \pi \gamma \cos (\theta)}$. The error in the tensile strength measurements corresponds to the confidence interval of the linear fit and will not be mentioned for the further tensile strength graphs.

\subsubsection{Experimental results}

The tensile strength measurements are very sensitive to the capillary forces, since the friction component can be clearly separated. The tensile strength measurements as a function of the liquid content are shown in Figure 3.19 [79]. We observe at low liquid contents an increasing tensile strength. At larger liquid contents above $W=0.01$ we observe a plateau value of the tensile strength of approximately $350 \mathrm{~N} / \mathrm{m}^{2}$. The plateau height is in agreement with literature values when the literature values are inversely rescaled by the bead radius to our bead radius of $141 \mu \mathrm{m}$. Pierrat et al. [73] measured $350 \mathrm{~N} / \mathrm{m}^{2}$ and Richefeu et al. [74] measured $500 \mathrm{~N} / \mathrm{m}^{2}$. This centrifuge technique is limited, at our bead radii, to liquid contents below $W<0.2$ due to drainage of the liquid. This technique can probably be extended to larger liquid contents by using smaller beads. 


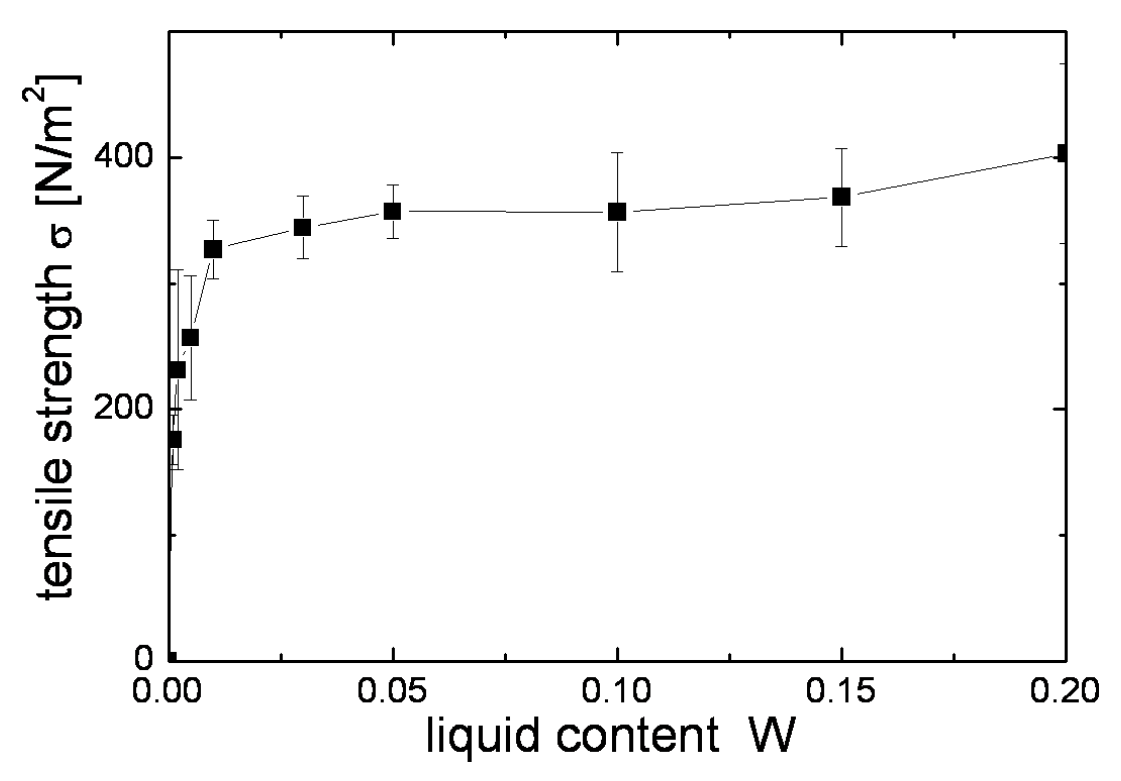

Figure 3.19: Tensile strength of glass beads with a radius $R$ of $141 \mu \mathrm{m}$ for various liquid contents [79].

The plateau values of the tensile strength can be basically predicted by the Rumpf model [93]. The idea is to count the number of capillary bridges in one slice within area $A$ and multiply them with the capillary force: $\sigma_{\text {Rumpf }}=\rho_{\text {packing }} N_{\text {bridges }} \gamma \cos (\theta) / 2 R . N_{\text {bridges }}$ is the number of capillary bridges on a bead. The Rumpf model gives $1000 \mathrm{~N} / \mathrm{m}^{2}$ for our bead radius, which is about 3 times larger than the experimentally measured values. The reason that a smaller force ruptures the pile is that a crack searches for the weakest points between the network of capillary bridges, and this decreases the tensile strength of a wet granulate.

In the Rumpf model [93] the tensile strength scales linearly with the surface tension $\gamma$ and is inversely proportional to the bead radius. We tested these scalings by using different liquids and by varying the bead size.

To test the dependence of the tensile strength on the surface tension $\gamma$ we used water, olive oil, and ethanol (cf. Table 3.1) as wetting liquids and the results are shown in Figure 3.20 a). We found that the tensile strength scales linearly with the surface tension in agreement with the Rumpf model and our experimental findings from the vertical fluidization measurements. The second scaling test of the tensile strength was the inverse dependence on the bead radius $R$, which is shown in Figure $3.20 \mathrm{~b}$ ). We used both cleaned beads (red dots) and beads as delivered (black dots) for radii in the range of $40 \mu \mathrm{m}$ to $300 \mu \mathrm{m}$. In both cases the data points 

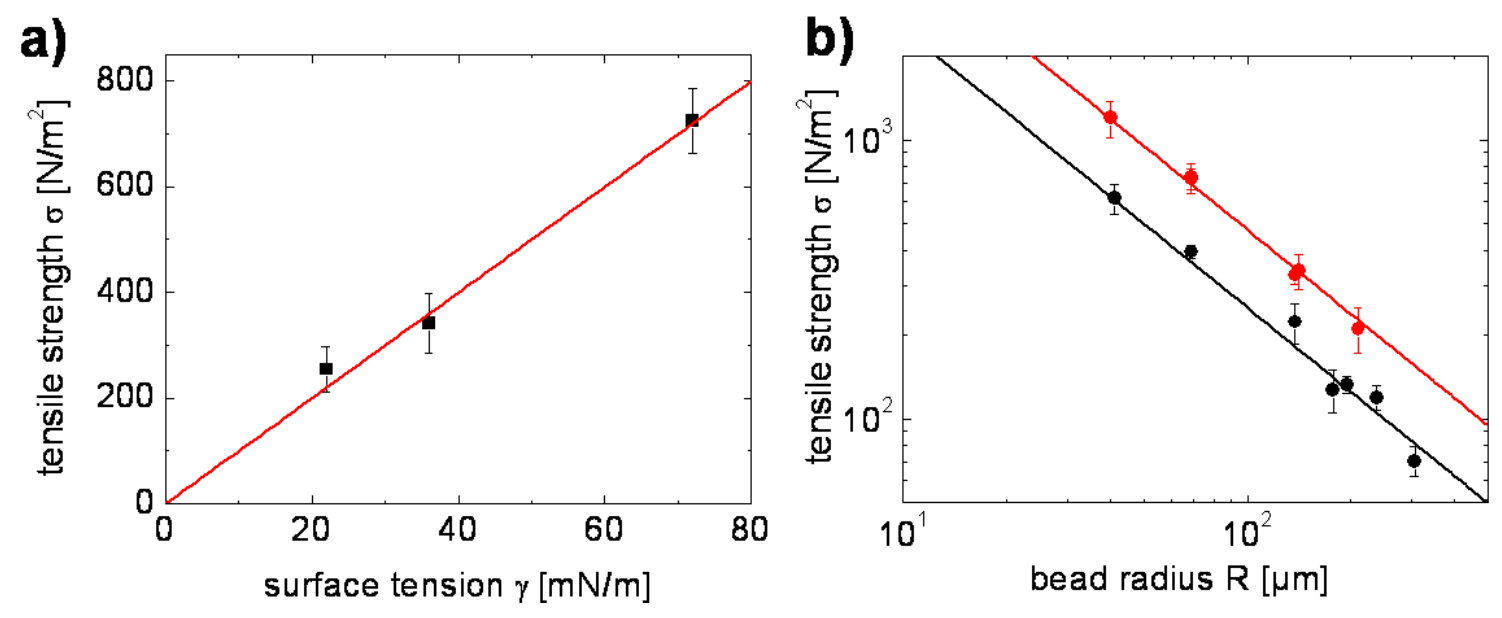

Figure 3.20: a) Tensile strength for the liquids ethanol, olive oil, and water with different surface tensions $\gamma$ for glass beads of $R=68 \mu \mathrm{m}$ and a liquid content $W=0.01$. b) Tensile strength as a function of the bead radius in a log-log plot. We used cleaned glass beads (red dots) and as delivered beads (black dots) in the range of $R=40 \mu \mathrm{m}$ to $300 \mu \mathrm{m}$ and a liquid content $W=0.01$.

follow the expected inverse proportionality of the bead radius dependence and the cleaned beads have nearly twice the tensile strength. It seems that the glass beads as they were delivered may lower the surface tension of the wetting liquid due to contamination, and as a result may have also increased the contact angle.

So far we have primarily used mixtures of glass beads and water where the liquid has a contact angle $\theta$ of around $0^{\circ}$ on the beads surface. When using other bead materials like basalt we can change the contact angle to $70^{\circ}$ which is expected to affect the tensile strength. In Figure 3.21 a) we compared the tensile strength of basalt beads to that of glass beads of the same radius of $68 \mu \mathrm{m}$ as a function of the liquid content. The tensile strength of glass beads (red dots) shows a plateau for liquid contents larger than $W>0.005$. The tensile strength of basalt beads has about the same value compared to glass beads at low liquid contents, but at $W>0.01$ a rapid decrease of the tensile strength is observed. In addition, the tensile strength of the basalt beads increases at lower liquid contents then with the glass beads. This is due to the roughness of the beads and was also observed with the critical fluidization acceleration and the shear cell techniques described previously. 

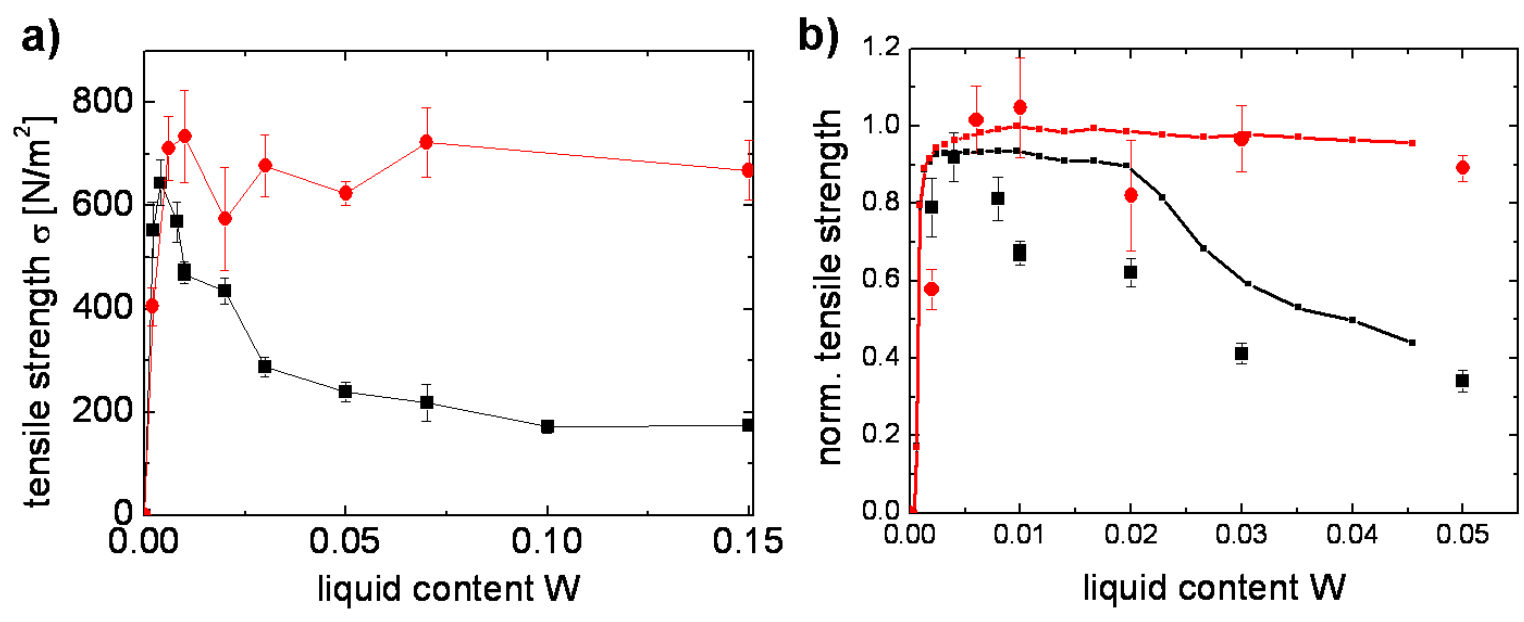

Figure 3.21: a) Tensile strength as a function of the liquid content of water for glass beads (red dots) with a contact angle of $0^{\circ}$ and basalt beads (black squares) with a contact angle of $70^{\circ}$ for the same bead radius $R=68 \mu \mathrm{m}$. b) Normalized tensile strength as a function of the liquid content for glass beads (red dots, $\theta_{\text {pile }}=0^{\circ}$ ) and basalt beads (black squares, $\theta_{\text {pile }}=70^{\circ}$ ) in comparison with calculated tensile strength values (red and black line). The effects of the pulled capillary bridges, the number of capillary bridges on a bead and the existence of open and compact clusters are taken into account in the calculated values.

The similar values for the tensile strength obtained for glass and basalt beads at low liquid contents can be explained by the fact that it takes more force to rupture stretched capillary bridges as discussed schematically in Figure 3.17

Possible explantation for the observed differences in the tensile strength at higher liquid contents between basalt and glass beads can be found in the number of capillary bridges and the existence of open and compact clusters and will be explained in chapter 4. A quick calculation should show that this effect exists. We start with cylindrical capillary bridges and calculate their liquid volumes and the respectively contact angles. Then the beads are pulled to the calculated normalized separation distance $S^{*}$ where the contact angle becomes $10^{\circ}$ (receding angle). The capillary force at $S^{*}$ and $\theta=10^{\circ}$ can be calculated from Eq. 1.8. This capillary force value is multiplied with the normalized number of capillary bridges on a bead calculated from Figure 4.4 and then plotted as normalized tensile strength as black line in Figure 3.21 b). For comparison is the calculated normalized tensile strength for the wetting case illustrated as red line. At large contact angles compact clusters have no attractive capillary forces as shown in end of the next chapter 4. A crack in the pile will always find the weakest path and if there are compact clusters, it will break through them. This makes the 


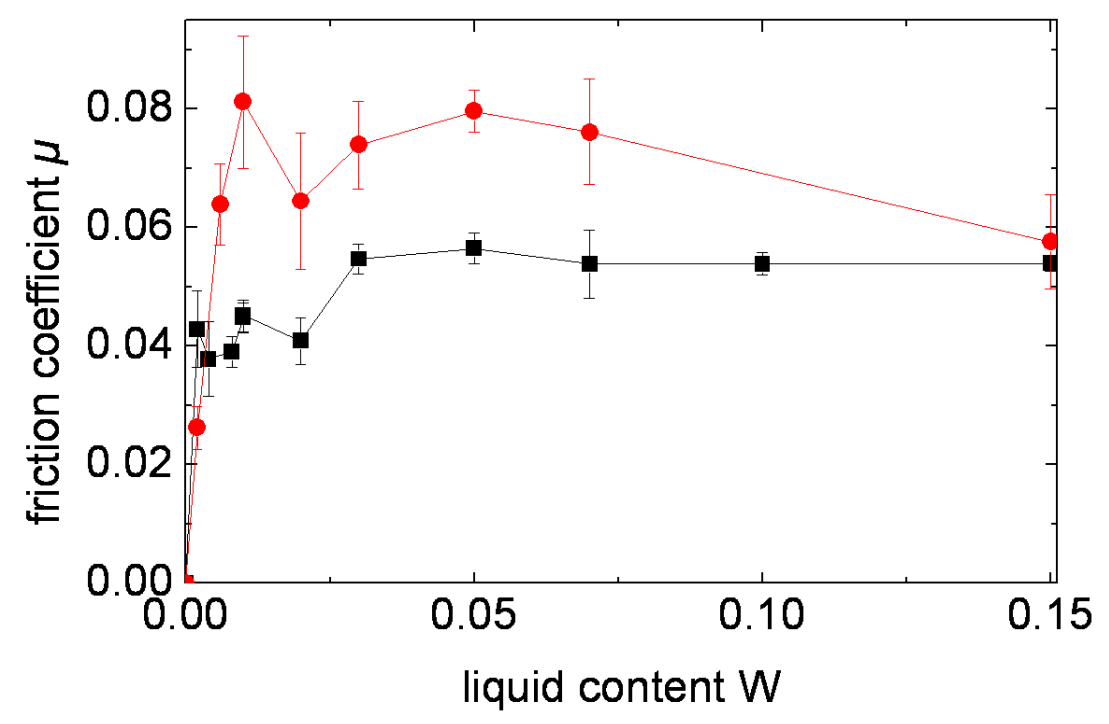

Figure 3.22: The friction coefficient $\mu$ of glass beads (red dots) and basalt beads (black squares) as a function of the liquid content.

tensile strength measurement very sensitive for the liquid morphology. This rough calculation of the pulled capillary bridges can be done much better with numerical minimization with the Surface Evolver [61].

Using the centrifuge setup we can measure the tensile strength in addition to the friction coefficient $\mu$ for capillary bridges between the beads or between beads and the container walls. In Figure 3.22 we show how the friction coefficient depends on the liquid content. At low liquid contents the friction coefficient increases, and at a liquid content of about $W>0.005$ peak values of about $\mu=0.07$ for glass beads and $\mu=0.05$ for basalt beads have been obtained.

A possible reason for this dependence might be that in the dry case the beads roll without sliding and therefore the friction coefficient is close to zero. With more liquid present in the sample the capillary bridges strengthen to give a constant attractive force as Halsey and Levine [51] have reported, and with the larger force present the friction coefficient $\mu$ increases. We have measured the friction coefficients of various bead radii and have found no size dependence in the range of 20 to $200 \mu \mathrm{m}$. 


\subsection{Comparisons}

We have used three measurement techniques to measure the properties of wet granulates; the critical vertical fluidization acceleration, the shear stiffness, and the tensile strength. All of the methods reveal the same generic behavior of wet granulates: the strength of the granulate increases dramatically for small liquid contents until a plateau is established and a further increase of the liquid content has little effect on the mechanical properties. At high liquid contents close to the fully submerged case the strength of the granulate decreases again. It is not clear yet why the merged capillary bridges into larger liquid morphologies at liquid contents of $W>0.02$ have no visible effect on the stiffness of the wet granulate.

For the basalt beads and water system, which has a large contact angle of $70^{\circ}$ between the beads and the liquid, differences were observed. The lower stiffness observed in the critical fluidization acceleration measurement could be explained by cosines $\theta$. The apparently contrarily larger stiffness measured with the shear cell can be explained by the effect of the pulled capillary bridges, an increased packing density compared with glass beads as well as the presence of a larger number of capillary bridges on a bead. The tensile strength measurements show the same pulling effect on capillary bridges, and the significant difference in the tensile strength at a liquid content $W>0.01$ could be explained by the number of capillary bridges on a bead and the presence of open clusters at low contact angles as well as compact clusters at large contact angles. The tensile strength measurement is very sensitive for the type of clusters, while a crack through the pile will find the weakest path. However, there should also be a visible decrease in the critical fluidization acceleration and the shear stiffness measurement, what is so far experimentally not observed. The different liquid morphologies and the liquid distribution present in a wet granulate is investigated using X-ray micro tomography and will be discussed in chapter 4 . 


\section{Chapter 4}

\section{Liquid distribution in a granular pile}

The addition of a wetting liquid to a dry granulate results in a remarkable increase in its' mechanical stiffness. In order to understand the underlying physical mechanism of the stiffening, we studied the distribution of the liquid and the packing geometry of the grains. The liquid content in the bead pile ranges from $W=0.005$ to $W=0.17$. The latter exceeds the quantity where only capillary bridges are present. The distribution of the liquid and the beads in random distributed beads piles is obtained using X-ray micro tomography techniques. In order to understand the influence of the contact angle of the liquid cluster formed in the granulate we have performed measurements using different liquids and bead materials.

\subsection{Liquid morphologies in a pile of beads}

Using X-ray micro tomography as introduced in chapter 2 we measured the liquid distribution in wet piles of randomly distributed beads of radius $R=140 \mu \mathrm{m}$ for various liquid contents. If not mentioned we prepared the samples with preparation method 1 which results in a nearly constant packing density of $0.57 \pm 0.01$. After preparation the samples were given several hours to equilibrate and to form the liquid morphologies discussed below. Slices through 3D tomographic images are shown in Figure 4.1 [79] for liquid contents of $W=0.01$, $W=0.03$, and $W=0.07$ respectively and bead radii $R=280 \mu \mathrm{m}$. At $W=0.01$ only capillary bridges, the liquid morphology between two beads, can be found in the sample. Whereas for a larger liquid content $W=0.03$ liquid clusters, the morphologies between three or more beads, 

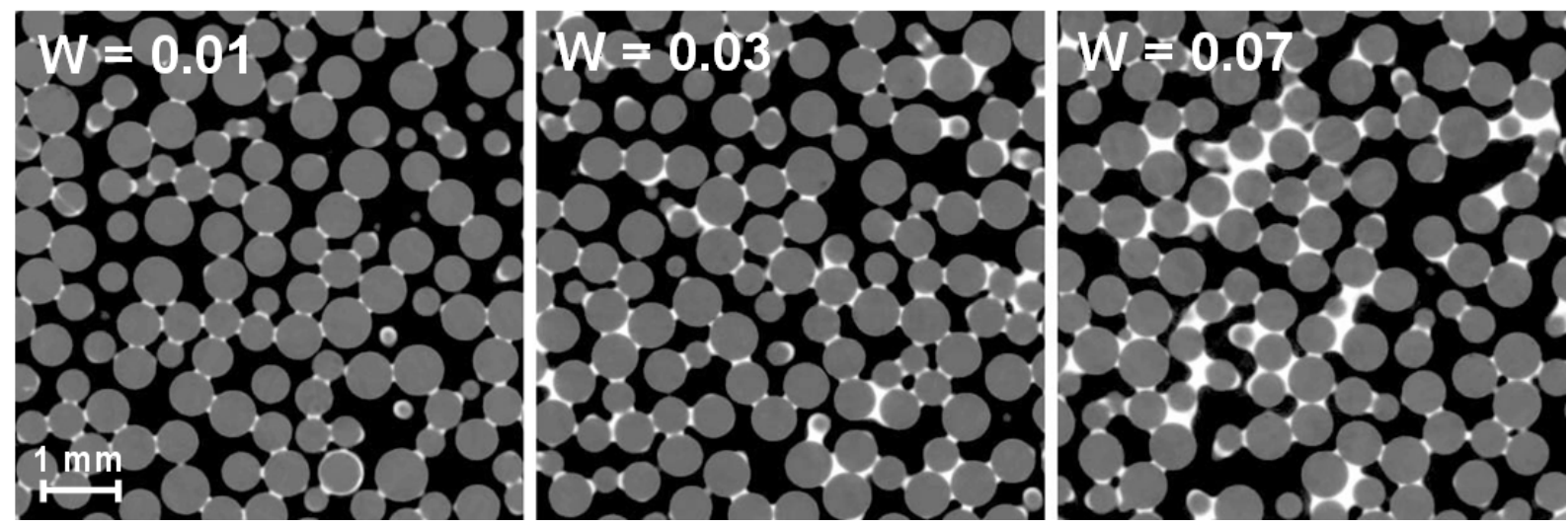

Figure 4.1: 2D slices through 3D tomographic images of randomly distributed glass beads with bead radii $R=280 \mu \mathrm{m}$ at various liquid contents $W=0.01, W=0.03$, and $W=0.07$ respectively [79].

are visible. For an even larger liquid content $W=0.07$, larger clusters between many beads have formed.

In the X-ray micro tomographic images we can clearly distinguish liquid capillary bridges and liquid clusters by their volume. Figure 4.2 shows a histogram of liquid capillary bridges and small liquid morphologies found in a bead pile with a bead radius $R=140 \mu \mathrm{m}$ and a liquid content $W=0.035$. The capillary bridge is the liquid morphology between two beads and a 3D capillary bridge is shown in Figure 4.2 top left [79]. The limited resolution of the X-ray micro tomography results in deviations from the real shape of the capillary bridges. For example capillary bridges are resolved as 'donuts' with a hole in the center. The diameter of this hole will decrease as the voxel resolution is increased. The smallest liquid cluster is formed between three beads. We named this cluster trimer, because a trimer has three contact points between the beads. A 3D trimer is illustrated in Figure 4.2 top [79]. The next cluster has five contacts between four beads and we named it pentamer. Between four beads, a tetrahedron gap, is also a tetrahedral cluster possible. It has six contacts points between four beads and we used the name tetrahedral cluster instead of 'hexamer'. A heptamer cluster has seven contacts between five beads. A larger 3D cluster is illustrated in Figure 4.2] top right [79].

The first volume peak in Figure 4.2 corresponds to capillary bridges with a volume of about 100 voxels. The second peak at 550 voxels can be identified as a trimer. A pentamer has a volume around 1000 voxels which is almost the volume of two trimers. The liquid morphologies corresponding to the further peaks are heptamers and filled tetrahedra gaps. All larger morphologies can not be identified just by their liquid volume but can be separated by the surface 

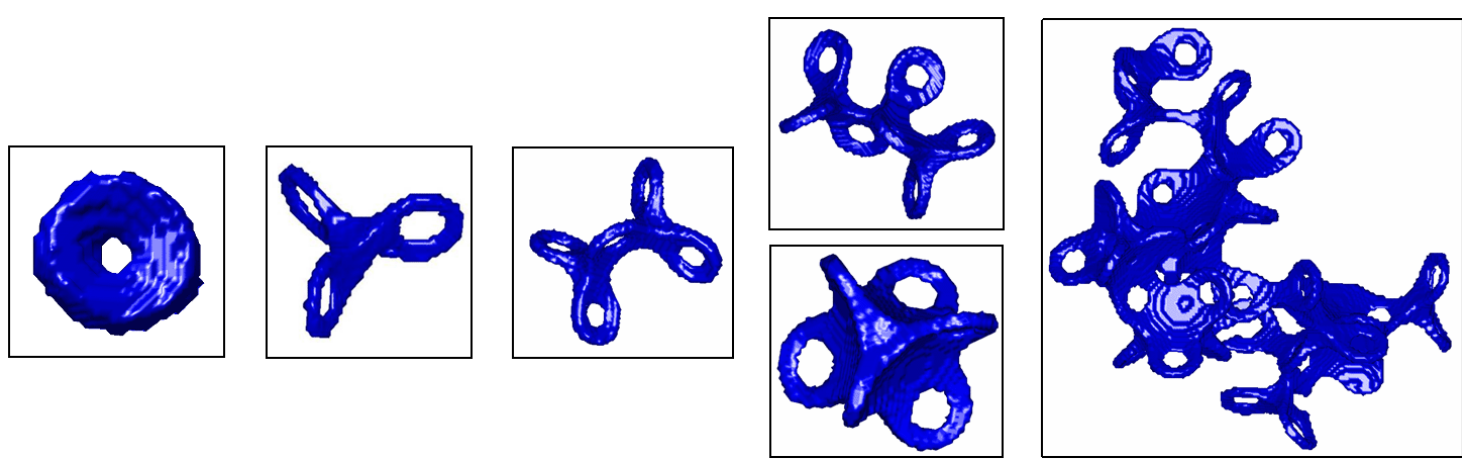

heptamers

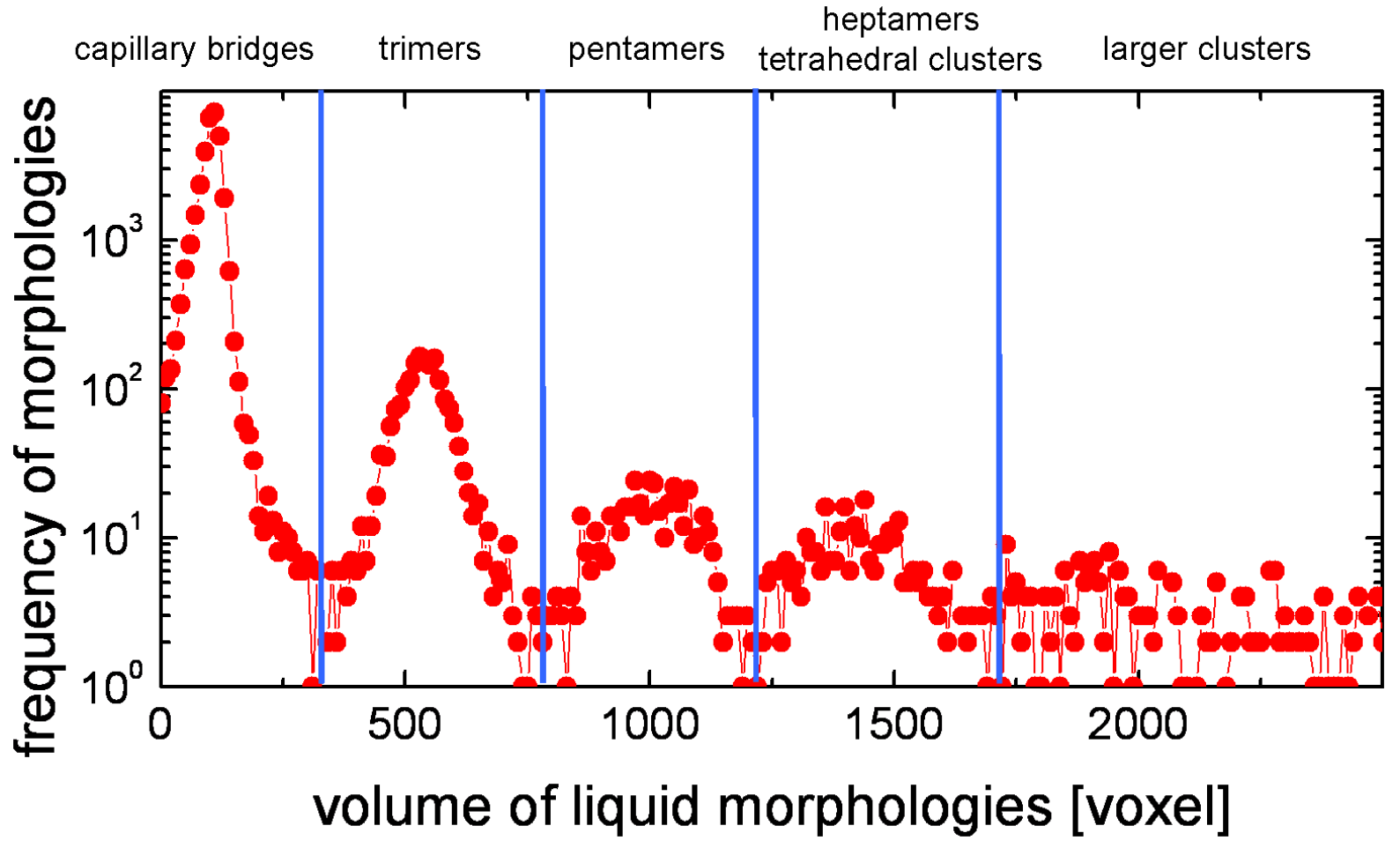

Figure 4.2: Histogram of the frequency of liquid morphologies as a function of their volume prepared for a liquid content $W=0.035$ and bead radius $R=140 \mu \mathrm{m}$. The volume peaks can be used to identify the liquid morphologies as shown in the top row as 3D clusters found in $\mathrm{X}$-ray micro tomographies [79]. 
area to volume ratio. The heptamers have a volume peak of 1450 voxels which is almost three times that of a trimer. A tetrahedral cluster contains about 1450 voxels which is more liquid than in a pentamer even if it has the same number of connected beads. We have also found clusters where a trimer was connected to a tetrahedral cluster. It seems that large clusters are built from trimers and tetrahedral clusters in a plane.

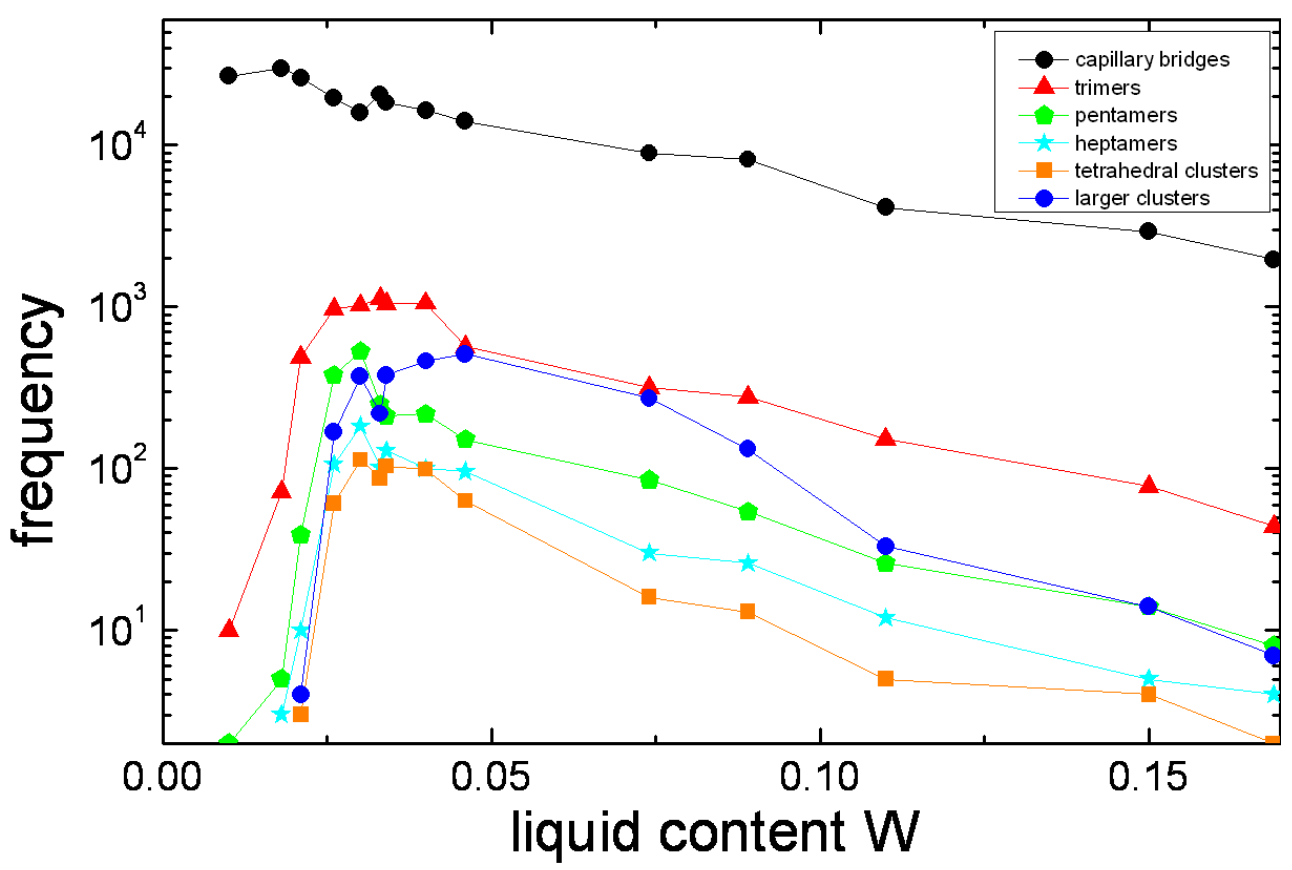

Figure 4.3: Frequency of capillary bridges and liquid clusters found in a pile of wet glass beads of $R=140 \mu \mathrm{m}$ for various liquid contents.

To explore the appearance of liquid morphologies for various liquid contents a histogram for each kind of the five smallest liquid morphologies is plotted, cf. Figure 4.3. The total number of capillary bridges in a sample decreases from 30000 at $W=0.01$ to only 2000 at $W=0.17$. Liquid clusters can be found above a critical liquid content. The critical liquid content of a trimer is $W_{\text {trimer }}=0.012 \pm 0.001$ while the number of trimers shows a maximum at $W=0.03$. Beside the capillary bridges the trimer is the most frequent morphology. Pentamers, heptamers, and tetrahedral clusters have a critical liquid content of $W=0.020 \pm 0.002$ and show a maximum of their frequency around $W=0.03$. The frequencies of clusters larger than heptamers and tetrahedral clusters are summed together. This number rises rapidly at a liquid content $W=0.025$ and shows a maximum at $W=0.045$. At larger liquid contents a very large 
cluster develops and at a liquid content $W_{p}=0.12 \pm 0.02$ the sample contains a percolating cluster [94]. However, this percolating cluster exists with a large number of capillary bridges and small clusters. This percolating cluster grows as the liquid content is further increased and finally all smaller clusters will be connected to the percolated cluster.

In the next sections we will examine the capillary bridges in more detail and later extend our analysis to larger liquid morphologies in section 4.5.

\subsection{Number of capillary bridges on a bead}

In granular piles of glass beads with liquid contents below $W=0.01$ only capillary bridges exist and no liquid cluster can be formed. Thus the stiffness of the wet bead pile is proportional to the number of capillary bridges on a bead. The larger the number of the capillary bridges the stiffer becomes the wet pile.

The number of capillary bridges on a bead can be estimated by the coordination number which is defined as the number of contacts between beads. The coordination number is derived from the 3D stability criteria for a pile of beads, where new beads are placed from top on a bed of beads. As a results a sphere has on average 6 contacts in the non friction case of an isostatic packing [95]. Only 4 contacts are enough for an isostatic packing with friction. The number of capillary bridges in experiments found at low liquid content is slightly larger than the coordination number, because capillary bridges can also be formed between beads with a small separation distance. A value close to 6 or 7 capillary bridges on a bead was also observed from Mason, Clark, and Bernal [96, 97, 98] in experiments where lacquer was added to a pile of beads. After drying the pile was broken into single beads and the number of capillary bridges was counted. Gröger et al. [99] investigated the number of capillary bridges as a function of the surface roughness in a simulated drying process and found 7.6 capillary bridges on a bead.

For our typical sample preparation the packing density of $\rho_{\text {packing }}=0.57 \pm 0.01$ was very reproducible as illustrated in Figure 2.11. We used this typical packing density for all further experiments. The number of capillary bridges as a function of the liquid content is shown in Figure 4.4 for a bead radius of $140 \mu \mathrm{m}$.

From optical microscopy measurements we found that the critical liquid content of the roughness regime, the formation of capillary bridges, is $W_{r_{c b}}=(6 \pm 2) \cdot 10^{-4}$ shown as orange dots in Figure 4.4 [100] and in well agreement with the value found for the critical fluidization 


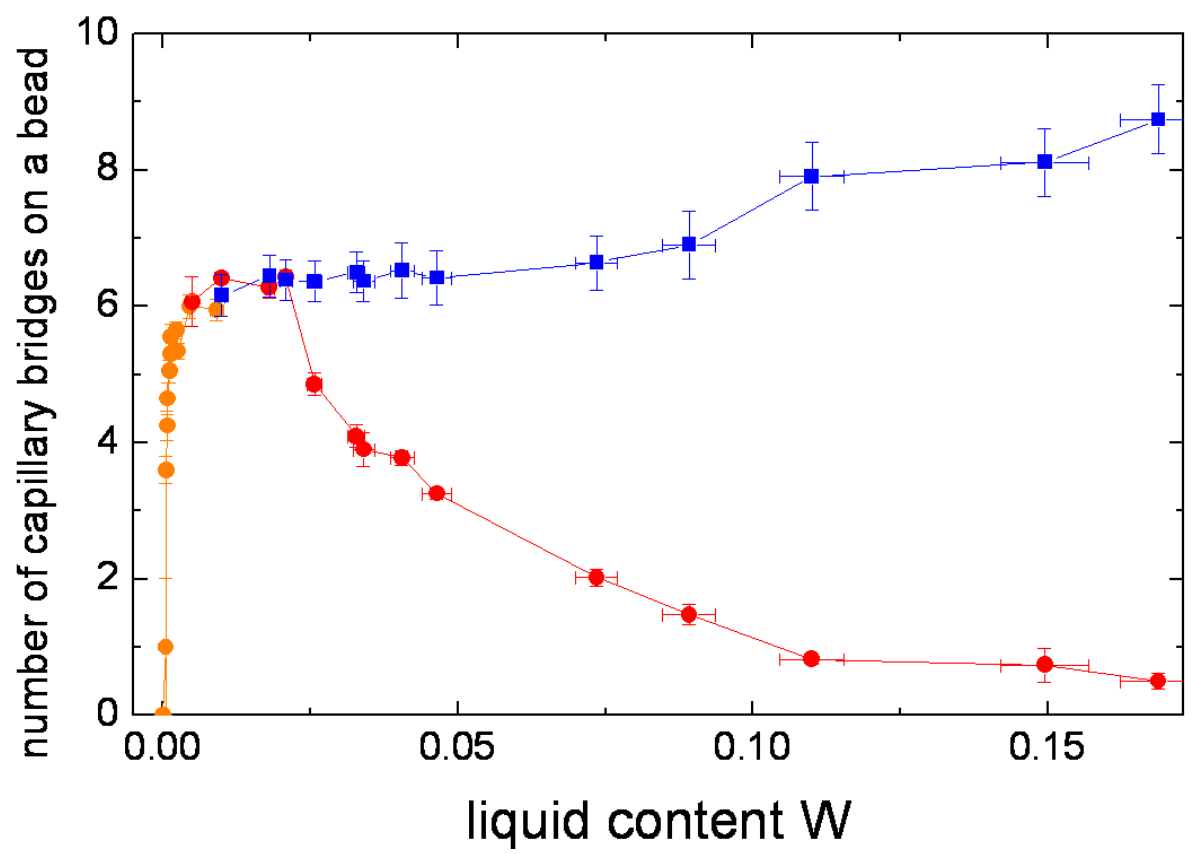

Figure 4.4: Number of capillary bridges and 'wet contacts' (blue squares) on a bead as a function of the liquid content derived by optical microscopy (orange dots) [100] and X-ray micro tomography (red dots) [62].

acceleration. At a liquid content of $W=0.01$ we observed that $6.5 \pm 0.3$ capillary bridges are present on each bead. With X-ray micro tomography we estimated the number of capillary bridges for larger liquid contents, shown as red dots in Figure 4.4 [62]. The critical liquid content where capillary bridges merge into clusters and the number of capillary bridges on a bead decreases is $W=0.025$ [62]. Even at a large liquid content of $W=0.17$ a certain number of isolated capillary bridges are still present in the sample.

At a liquid content $W>0.025$ the number of capillary bridges on a bead decreases, since capillary bridges merge into liquid clusters. We want to take these 'wet contacts' between beads in liquid clusters into account. A 'wet contact' exits between two beads whenever their distance is smaller then half a bead radius and they are wetted by the same liquid cluster. In this definition a capillary bridge counts as one wet contact and is shown in Figure 4.4 as blue squares. For larger liquid contents it becomes increasingly difficult to count the wet contacts since the beads connected to the same liquid cluster can have a large separation distance. The number of wet contacts rises slowly from 6 to 7 at a liquid content of $W=0.10$, which is very close to the percolation of the liquid. As the liquid content is further increased the number of wet contacts increases more rapidly. At the largest liquid content of $W=0.17$ we counted on 


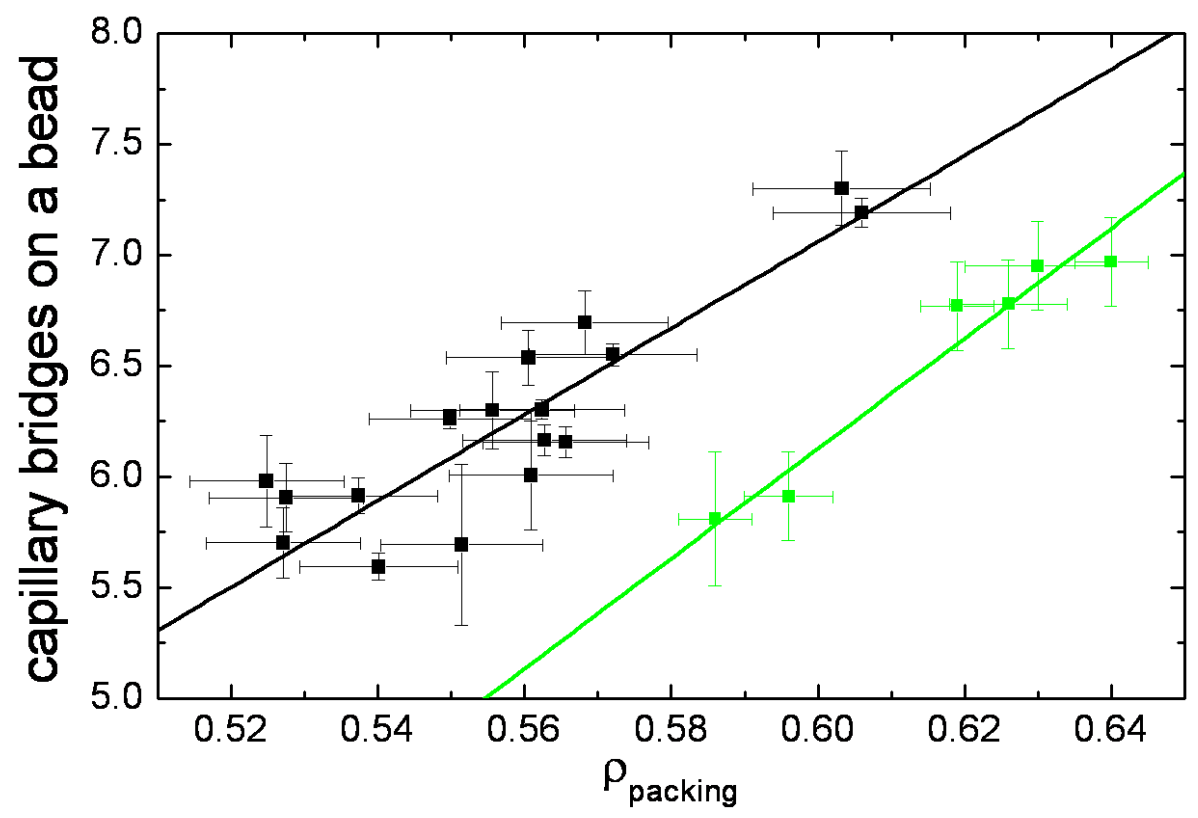

Figure 4.5: The average number of capillary bridges on a bead (black squares) for various packing densities in comparison with the average number of contacts (green squares) in a pile of dry beads investigated by T. Aste et al. [92]. Both data sets follow a linear dependence as shown by solid lines.

average $8.7 \pm 0.5$ wet contacts per beads.

To explore, how the number of capillary bridges on a bead varies with the packing density we prepared samples with a fixed liquid content of $W=0.01$. On the one hand, we know that capillary bridges are the only liquid morphologies in the sample at this liquid content, while on the other hand the liquid content is large enough to ensure that all contact points host fully developed capillary bridges. The packing density was varied from $\rho_{\text {packing }}=0.53$ to $\rho_{\text {packing }}=0.61$ using glass beads with an average radius of $R=280 \mu \mathrm{m}$. The number of capillary bridges on a bead was extracted from tomography images shown in Figure 4.5 as black squares. The number of capillary bridges on a bead increases linearly with the packing density and ranges between 5.6 and 7.3. A linear fit to the number of capillary bridges on a bead yields a slope of $19.4 \pm 1.0$ and a Y-axis offset of - $4.6 \pm 0.6$.

For comparison we plotted in Figure 4.5 the number of contacts on a bead for a random bead pile derived by X-ray tomography investigated by T. Aste et al. [92]. Obviously the number of dry contacts is smaller than the number of capillary bridges which can be spanned between separated beads. 


\subsection{Equilibration of liquid morphologies}

When the agitation of e.g. a vertically fluidized granulate is stopped, a certain time is needed for the liquid morphologies to equilibrate. During this process small capillary bridges will grow on the cost of larger capillary bridges. This equilibration comes to an end, if all capillary bridges have the same Laplace pressure. We explored the equilibration with the help of the fast X-ray micro tomography.

Our sample consist of glass beads with a radius of $280 \mu \mathrm{m}$ filled in a glass vial with an inner diameter of $10 \mathrm{~mm}$. We used an aqueous $\mathrm{ZnI}_{2}$ solution $\left(\gamma=75 \mathrm{mN} / \mathrm{m}, \eta=1 \mathrm{mPa} \mathrm{s}, \theta<10^{\circ}\right.$ on the glass beads, density of $1.3 \mathrm{~g} / \mathrm{ml}$ ). The sample vial was mounted on a lightweight shaker on top of the tomography rotation stage of ID15A as shown in Figure 2.5. A sinusoidal acceleration of more than $20 \mathrm{~g}$ was applied to fluidize the wet granulate. The agitation was stopped and with the shortest possible delay a time series of tomographies was recorded to capture the equilibration. Figure 4.6, a) shows the volume of individual capillary bridges as a function of the time elapsed after the agitation has been stopped [79]. The data sets show the evolution of capillary bridges averaged over a narrow volume range. The volume of small capillary bridges increases while the volume of large capillary bridges decreases with time. The equilibration time $t_{\text {equi }}$ obtained by an exponential fit for small and large capillary bridge volumes was found to be $(100 \pm 30) \mathrm{s}$.

Histograms of the normalized frequency of capillary bridges as a function of the volume are shown in Figure 4.6 b) at three points in time after the vertical agitation has been stopped. After $10 \mathrm{~s}$ we find a monotonous decaying distribution of the capillary bridges for increasing volume. After $350 \mathrm{~s}$ a maximum has developed at a volume of 230 voxels. At large times, in this case around $1300 \mathrm{~s}$ after stopping the agitation the distribution does not change noticeably. In this final distribution the maximum has shifted to a volume of 270 voxels while the maximum has decreased its half width. Due to the polydispersity (10\%) and the asphericity (aspect ratio $1.02 \pm 0.01$ ) of the beads employed in the experiments a certain minimal width of the volume maximum can be expected. A comparison of the data sets for glass beads and ruby beads, however, shows a paradox trend: the width of the volume maximum for ruby beads is much broader than the volume peak for glass beads, although the polydispersity and asphericity of the ruby beads is at last one order of magnitude smaller than for glass beads. 

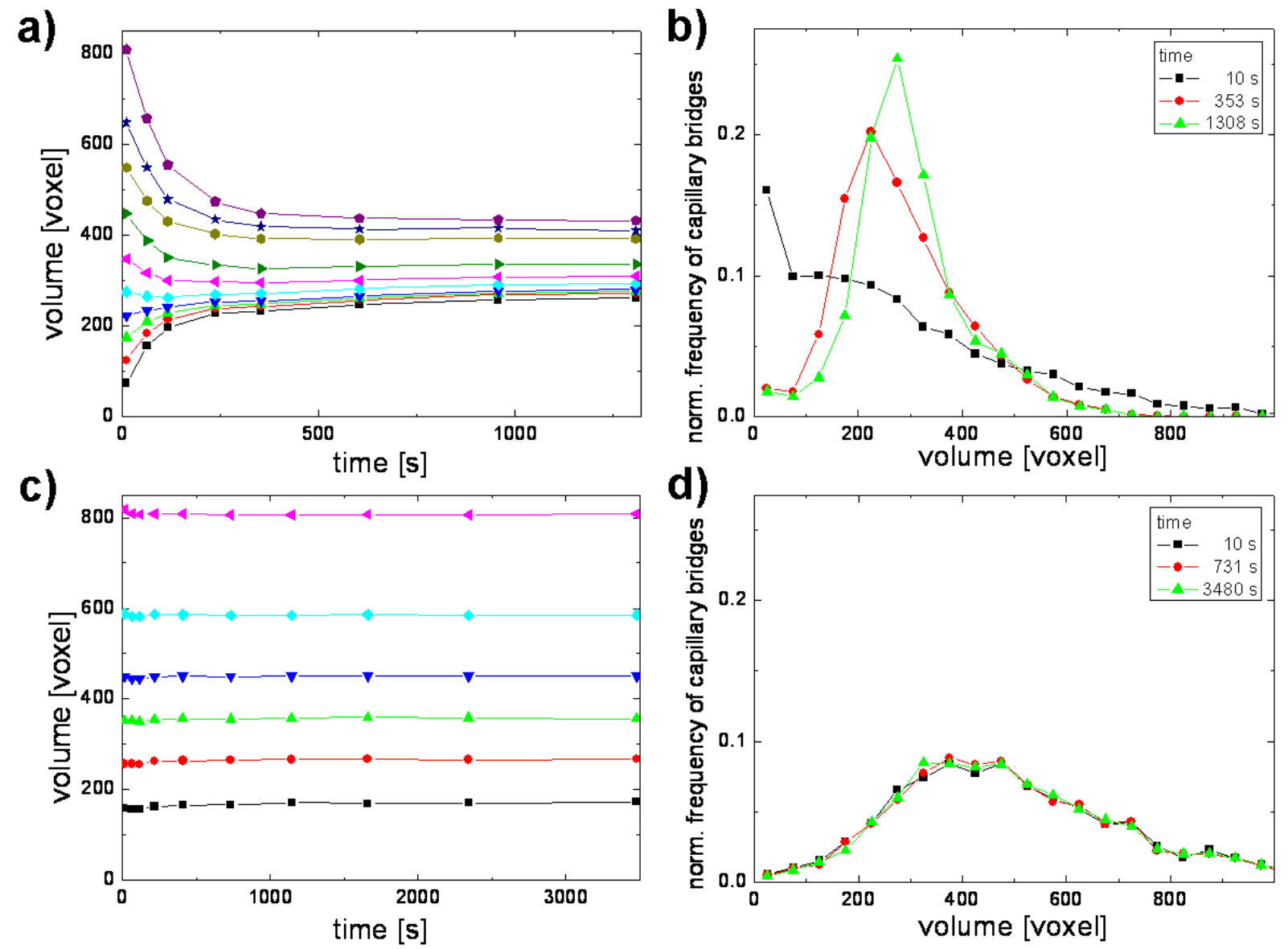

Figure 4.6: Equilibration of capillary bridges in a wet granulate after vertical agitation at a liquid content of $W=0.02$. Samples a) and b) are made of glass beads with an average radius of $280 \mu \mathrm{m}$ while samples c) and d) consist of ruby beads with $300 \mu \mathrm{m}$. a) Volume of capillary bridges as a function of the time elapsed after the agitation has been stopped [79]; and b) normalized frequency of the capillary bridge volume at three times. In c) and d) the same analysis is shown for ruby beads. 
The driving force for the equilibration is the Laplace pressure of the liquid. In case of a wetting liquid, i.e. with a hydrophilic contact angle, small capillary bridges exhibit a smaller Laplace pressure than larger capillary bridges. This pressure difference between small and large capillary bridges force small capillary bridges to receive volume from the large capillary bridges. During this volume exchange, the Laplace pressure of the individual capillary bridge will approach a constant value. The equilibration will end, once the Laplace pressure is the same in all liquid structures.

We repeated the equilibration experiment using the same aqueous salt solution but added a small quantity of dishwasher surfactant. The surfactant lowers the surface tension of the salt solution from about $\gamma=75 \mathrm{mN} / \mathrm{m}$ to about $\gamma=50 \mathrm{mN} / \mathrm{m}$. Using this system, we measured an equilibration time of $t_{\text {equi }}=(130 \pm 30) \mathrm{s}$.

A possible reason for the longer equilibration time with surfactant is the lower driving force because the Laplace pressure scales linearly with the surface tension. Also possible is a reduced thickness of the liquid surface film.

Obviously a certain mechanism that allows for the transport of liquid volume is required to explain the pressure equilibration of capillary bridges. This exchange may proceeds via a thin wetting layer on top of the beads. For volatile liquids, i.e. with a low vapor pressure an additional volume transfer can occur by diffusion through the ambient vapor phase.

To explore the effect of the contact angle on the time scale of equilibration we performed a series of experiments using less hydrophilic ruby beads instead of glass beads. The ruby spheres had a radius of $300 \mu \mathrm{m}$ while the contact angle with the salt solution was $55^{\circ} \pm 10^{\circ}$. Large contact angles will likely decrease the thickness of the liquid film or suppress its formation. In both cases we expect an increase of the equilibration time.

Figure 4.6 c) shows the volume of individual capillary bridges between ruby beads as a function of the time elapsed after agitation has been stopped. The volume of all capillary bridges remains constant in time.

Figure 4.6 d) shows histograms of the normalized frequency of capillary bridges of certain volume for three different times after agitation has been stopped. The histogram taken after $10 \mathrm{~s}$ shows a maximum frequency at a finite volume in contrast to the corresponding histogram for glass beads in Figure $4.6 \mathrm{~b}$ ). In contrast to the time series for glass beads the distribution of volumes does not change with time. Compared to the glass beads, the peak of the distribution 
of volumes is decreased by a factor of three while the half width of the maximum is three times wider.

Using ruby beads and the standard $\mathrm{ZnI}_{2}$ solution we observed only small changes in the volume of the capillary bridges. This can be viewed as a strong indication for the suppression of the main transport mechanism which is present for $\mathrm{ZnI}_{2}$ solutions on glass beads. Therefore we conclude that the liquid transport occurs in our system via a surface film.

We want compare the equilibration time $t_{\text {equi }}$ derived from several experiments using different liquids. We assume that the equilibration time scales inversely proportional with the surface tension $\gamma$ and linearly with the viscosity $\eta$. The following expression takes this two factors into account for a liquid $\mathrm{A}$ and a liquid $\mathrm{X}$ which equilibration time should be scaled to the parameters of liquid A.

$$
t_{\text {equi_scaled_A }}=t_{\text {equi_X }} \cdot \frac{\gamma_{X}}{\gamma_{A}} \cdot \frac{\eta_{A}}{\eta_{X}}
$$

The Eq. 4.1 is a first order approximation without taking effects into account like varied film thickness, bead radius, surface roughness, and contact angles.

For the equilibration time experiments we used an aqueous $\mathrm{ZnI}_{2}$ salt solution with a density of $1.3 \mathrm{~g} / \mathrm{ml}$ and label this liquid as $\mathrm{ZnI}_{2} \mathrm{~A}$. The same salt solution with dishwasher surfactant is labelled as $\mathrm{ZnI}_{2}$ B. The in Scheel et al. [62] used $\mathrm{ZnI}_{2}$ salt solution with a density of $2 \mathrm{~g} / \mathrm{ml}$ is labelled as $\mathrm{ZnI}_{2} \mathrm{C}$ and the found equilibration time $t_{\text {equi }}$ was $300 \mathrm{~s}$. The from Kohonen et al. [100] used liquid squalene gave an equilibration time $t_{\text {equi }}$ in the range of $2-16 \mathrm{~h}$ and had observed that capillary bridge increases there volume in an equilibration process [100, 101].

\begin{tabular}{|c|c|c|c|c|c|}
\hline liquid & $R[\mu \mathrm{m}]$ & $\gamma[\mathrm{mN} / \mathrm{m}]$ & $\eta[\mathrm{mPa} \mathrm{s}]$ & $t_{\text {equi }}[\mathrm{s}]$ & scaled $t_{\text {equi_scaled }}[\mathrm{s}]$ \\
\hline$\overline{\mathrm{ZnI}}{ }_{2}, \mathrm{~A}$ & 280 & $\overline{75}$ & $\overline{11}$ & $\overline{100 \pm 30}$ & $\overline{c 100 \pm 30}$ \\
\hline $\mathrm{ZnI}_{2}, \mathrm{~B}$ & 280 & 50 & 1 & $130 \pm 30$ & $85 \pm 20$ \\
\hline $\mathrm{ZnI}_{2}, \mathrm{C}$ & 390 & 75 & 2 & $300 \pm 100$ & $150 \pm 50$ \\
\hline ualene [] & 570 & 30 & 30 & $(7-58) \cdot 10^{3}$ & $90-770$ \\
\hline
\end{tabular}

Table 4.1: Comparison of the equilibration time of several liquids to the liquid A scaled equilibration time by Eq. 4.1 for various liquids, bead radii, surface tensions, and viscosities.

Table 4.1 show the liquid parameters viscosity and surface tension, the bead radii, and equilibration times of four experiments. The to the liquid A scaled equilibration time for the four liquids shows the same value in respect of the error range. 

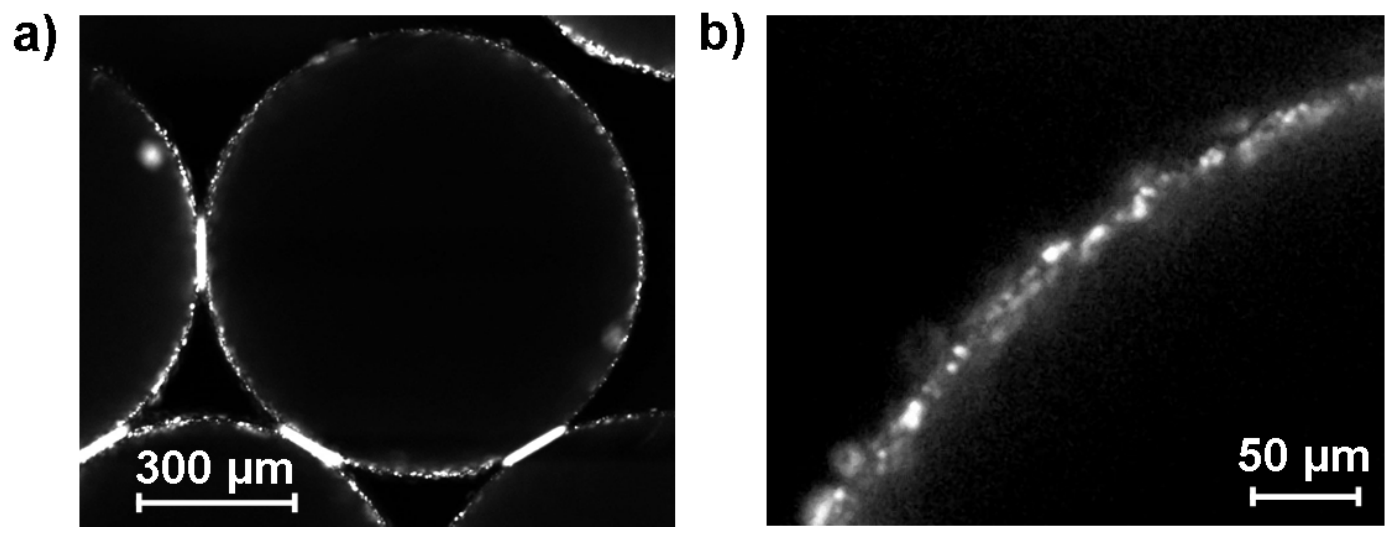

Figure 4.7: High resolution image of a fluorescence liquid on the surface of glass beads with radii of $390 \mu \mathrm{m}$ and a liquid content $W=0.01$ obtained by a confocal microscope. a) Show a slice threw a glass bead and b) a small part of the bead surface. The fluorescence liquid is visible in bright spots.

Since our transport happens via the liquid surface film on the beads we want to estimate its thickness. We used water with Fluorescein dye and homogenously mixed the liquid with glass beads with radii of $390 \mu \mathrm{m}$. The surface of the wetted beads was imaged with a confocal microscope. Since the sample contains glass beads, air, and water with different refractive index the image resolution of the confocal microscope is reduced. Figure 4.7 a) shows a high resolution image of the surface layer at liquid content of $W=0.01$. The fluorescence liquid is visible as bright spots as shown in Figure $4.7 \mathrm{~b}$ ). The thickness of the film is smaller than the height resolution of $0.8 \mu \mathrm{m}$ of the confocal microscope.

Tegzes et al. [102] measured the angle of repose for small liquid contents and saw an increase of the angle already at $10 \mathrm{~nm}$ large oil films.

We assume that the wetting film have a thickness in the range of $10 \mathrm{~nm}$ to $0.8 \mu \mathrm{m}$. Future measurements with more advanced measurement techniques will be needed to explore the film thickness. 


\subsection{Laplace pressure in a pile of wet beads}

In the section 4.1 we have discussed the spectrum of liquid morphologies which form in a granular pile of spherical beads. Time resolved measurements reveal that capillary bridges and larger liquid structures exchange volume due to differences in their Laplace pressure. Hence, we assume that for aqueous $\mathrm{ZnI}_{2}$ solutions on glass beads all liquid structures exhibit the same Laplace pressure after equilibration. Hence, the capillary bridges can be used as a pressure probe to determine the equilibrated Laplace pressure in the pile for various liquid contents. The geometry of capillary bridges between spherical beads of equal radius is well known, see, e.g. Refs. [34, 46, 57]. Using the calculated Laplace pressure of a capillary bridge as a function of liquid volume we are able to determine the Laplace pressure of an individual capillary bridge from its tomography image. The Laplace pressure should be identical not only for the set of capillary bridges but also for larger liquid morphologies. We derived the Laplace pressure (LP) in samples consisting of glass beads with radius $R=140 \mu \mathrm{m}$ and for liquid contents ranging from $W=0.005$ to $W=0.17$, as shown in Figure 4.8 a) [62].

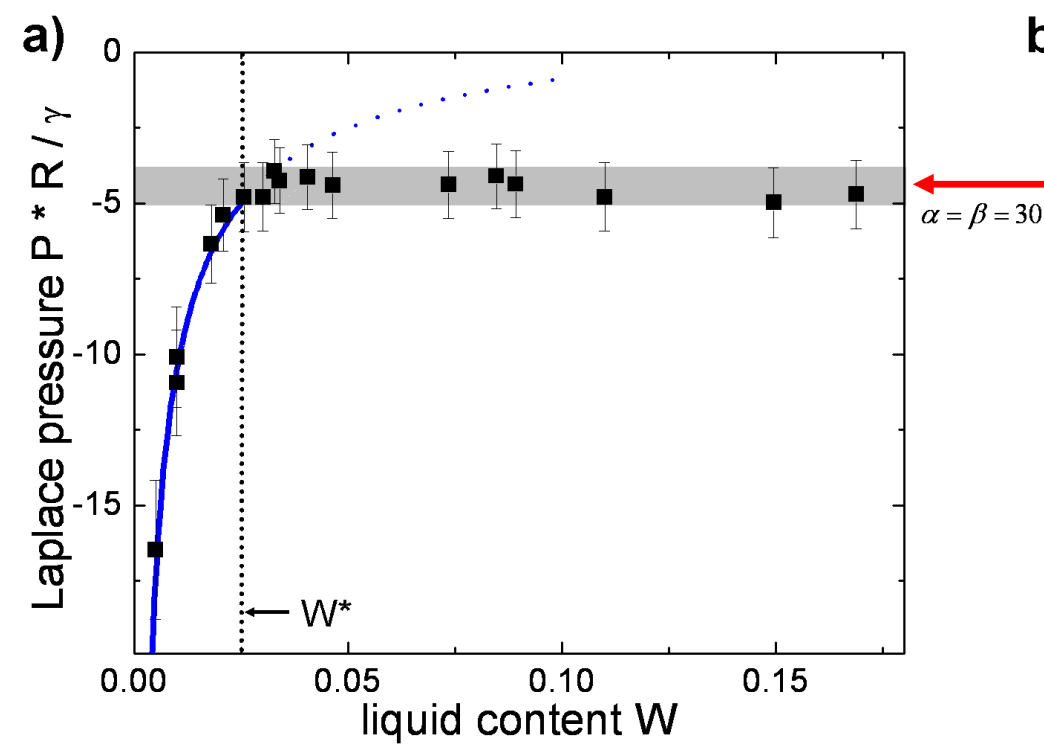

b)

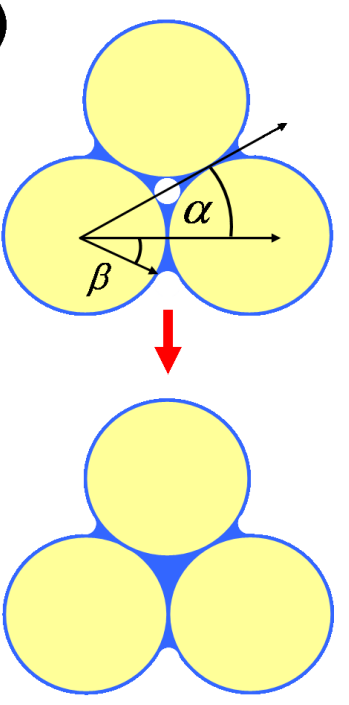

Figure 4.8: a) Normalized Laplace pressure of capillary bridges in a random bead pile as a function of the liquid content [62]. The blue line denotes the normalized Laplace pressure of capillary bridges derived by numerical minimization [60]; the gray bar indicates the critical normalized Laplace pressure, where three capillary bridges merge into a cluster, for the contact angle range between $0^{\circ}$ to $15^{\circ}$. b) Schematic of three beads in contact with an opening angle $\alpha=30^{\circ}$. Top: three capillary bridges with a half opening angle $\beta$. Bottom: a trimer consisting of three merged capillary bridges. 
The Laplace pressure of the capillary bridges is normalized by the bead radius $R$ and the surface tension $\gamma$ and is compared with the blue line of the normalized LP of capillary bridges derived by numerical minimization from M. Brinkmann [60] using the software package Surface evolver [61]. At low liquid contents the normalized LP of the capillary bridges derived from X-ray micro tomography (black squares) follows the blue line indicating the numerically obtained values for increasing liquid volume. At a critical liquid content $W^{*}=0.024$ [62] the experimentally determined normalized LP becomes constant with a value of $-4.5 \pm 0.3$. The deviation from the dotted blue line indicating the LP of a capillary bridge can be explained by the formation of trimers, cf. section 4.1.

The trimers represents the most frequently found clusters in our wet grain samples over a large range of liquid contents. Figure 4.8 b) shows a schematic of an 'ideal' three beads configuration with an opening angle $\alpha=30^{\circ}$. In the top view three capillary bridges are shown where the half opening angle $\beta$ of the capillary bridges becomes equal to the opening angle $\alpha$ of the beads. At this point the three capillary bridges merge into an trimer as shown in Figure $4.8 \mathrm{~b}$ ) bottom. The coalescence of the three capillary bridges $\beta=30^{\circ}$ occurs at the critical normalized LP value of -4.46 . For a contact angle of $0^{\circ}$ we find the simple expression [62]:

$$
P_{\text {max }}=-(1+2 \sqrt{3}) \gamma / R
$$

$P_{\text {max }}$ is shown as grey bar in Figure 4.8 a) for contact angles ranging from $0^{\circ}$ to $15^{\circ}$. The coalesced trimer has a smaller normalized LP as compared to the capillary bridges before coalescence, as will be discussed in more detail in the following section. After coalescence the trimer increases its volume by an exchange of volume with its neighboring bridges or clusters. Note, that the shape of a trimer and that of three capillary bridges before coalescence is almost identical provided the Laplace pressure is the same for both liquid morphologies. The only difference between these morphologies is the central opening of the triangular bead configuration being either filled or empty. A trimer can coalesce further with neighboring capillary bridges belonging to other triangular bead configurations forming pentamers, heptamers and even larger liquid clusters. All of these liquid morphologies are build of trimer subunits, i.e. the with liquid filled volume between three beads. The coalescence of these morphologies including capillary bridges will occur at essentially the same LP as given by the geometric rule in an ideal triangular bead configurations. 


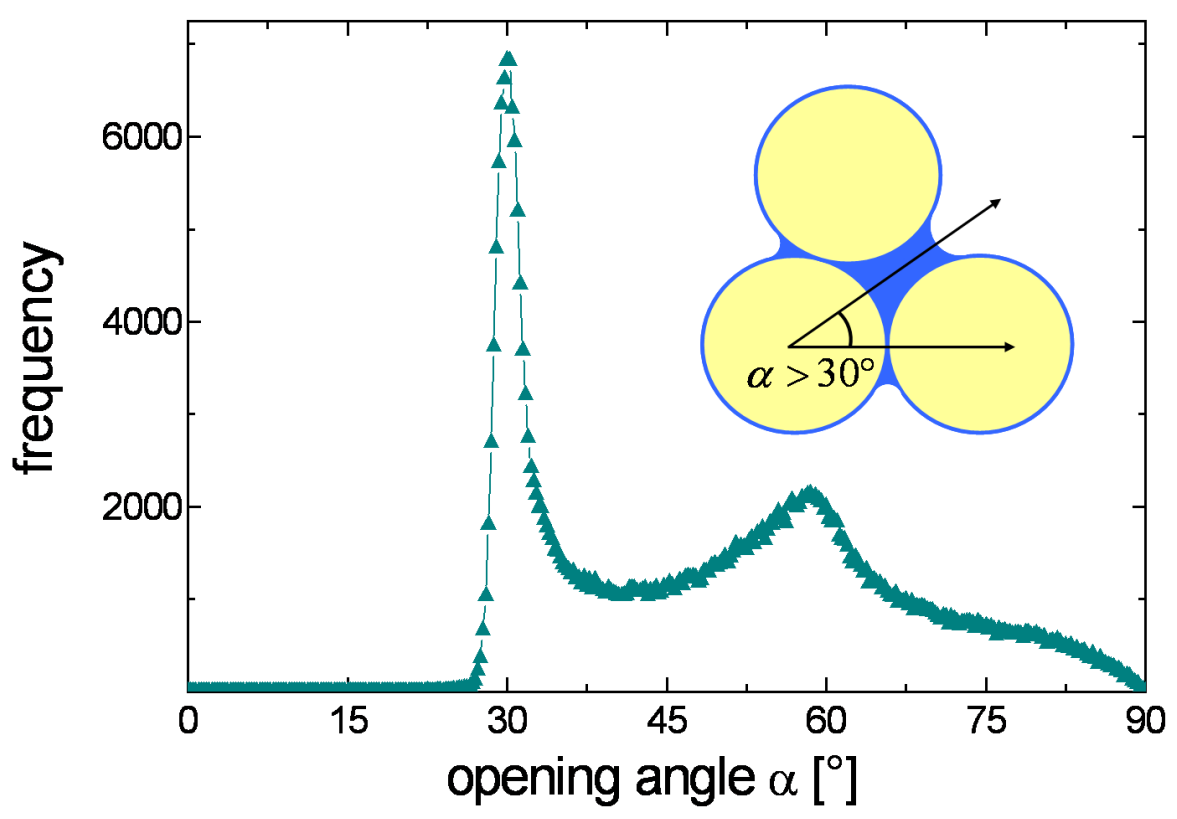

Figure 4.9: Frequency of pairs of contact points on a bead as a function of the opening angle $\alpha$ in a random bead pile [79]. In the inset is a trimer between beads with an opening angle $\alpha>30^{\circ}$ illustrated.

In a random bead pile, however, the triangular bead configurations exhibit a certain distribution of the opening angles $\alpha$. Figure 4.9 [79] shows the frequency of pairs of contact points on a bead as in our random bead pile as a function of the opening angle $\alpha$. In a generic configuration in a random bead pile not all three beads can be found in mutual contact which results in an increased opening angle $\alpha>30^{\circ}$, as depicted in the schematic inset in Figure 4.9 The frequency of pairs of contact points displayed in Figure 4.9 show a large 'peak' at $30^{\circ}$, i.e. the ideal bead configuration where all three beads are in contact. In addition, counts for smaller values than $30^{\circ}$ are an effect of not perfectly round glass beads. The smaller 'peak' close to an opening angle of $60^{\circ}$ can be explained as a pair of next nearest neighbor contact points.

If we condense the wetting liquid in a dry granular pile, the capillary bridges will form exclusively at the contact points. In the course of condensation the capillary bridge increase their half opening angle $\beta$ until it reaches the first peak in Figure 4.9. As the capillary bridges grow further and, hence, their half opening angle $\beta$, a larger number of capillary bridges in triangular configurations with $\alpha<\beta$ will transform into trimers. 
If we evaporate the wetting liquid from our granular pile the trimer units will break at a normalized Laplace pressure being smaller than the normalized LP at coalescence of three capillary bridges in the respective triangular bead configurations. The hysteresis in the Laplace pressure as well as the volume transitions between clusters and capillary bridges will be discussed in the next section.

\subsection{Volume transitions of liquid clusters}

Driven by the Laplace pressure the typical equilibration time for water was 2 minutes. Hence, after half and hour one can safely assume that the Laplace pressure has equilibrated and is identical for all liquid morphologies in the sample. Moreover, the Laplace pressure reaches a constant value as soon as $W>0.025$, i.e. the liquid content is large enough for liquid clusters to be formed. As we explained in the last section, this plateau of the Laplace pressure is a result of the cluster formation.

In this section we determine the Laplace pressure of capillary bridges, trimers and larger liquid morphologies from the measured mean curvature of the liquid/air interface as described in chapter 2. We present a detailed analysis for ideal trimers $\left(\alpha=30^{\circ}\right)$ and trimers with a separation distance between two of the three beads $\left(\alpha>30^{\circ}\right)$. For our wetting experiments we employed monodisperse ruby beads placed on a microscopy slide and took time series of optical microscopy images. Aqueous $\mathrm{ZnI}_{2}$ solution with added dishwasher surfactant was used to decrease the contact angle hysteresis on the ruby surface. The volume of the liquid morphologies can be controlled by evaporating and condensing liquid from the vapor phase. In this way, we were able to study the hysteresis related to the formation and decay of certain liquid morphologies in various bead geometries.

\subsubsection{Transition between trimer and capillary bridges in an ideal trian- gular bead geometry}

Increasing the liquid volume of three capillary bridges in an ideal triangular bead configuration (same radius, all beads in contact), a trimer is formed once the Laplace pressure reaches the critical value $P_{\max }$, cf. Eq. 4.2. Here, we assume that volume can be exchanged such the Laplace pressure is the same in all capillary bridges. If we evaporate liquid the trimer breaks 
into three capillary bridges at another critical Laplace pressure $P_{\min }$, as shown in the schematic of Figure 4.10 a). The experimental results will be compared with numerical minimizations of the interfacial energy in triangular bead configurations performed by M. Schwamberger and M. Brinkmann [54, 60].

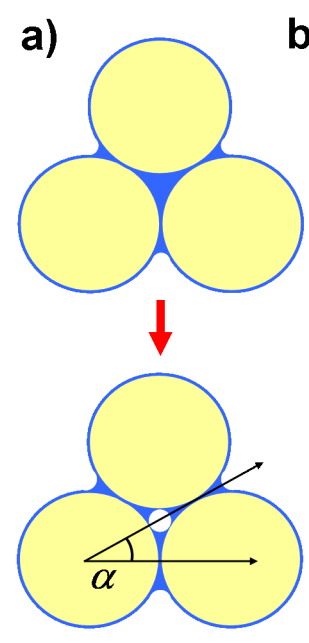

b)

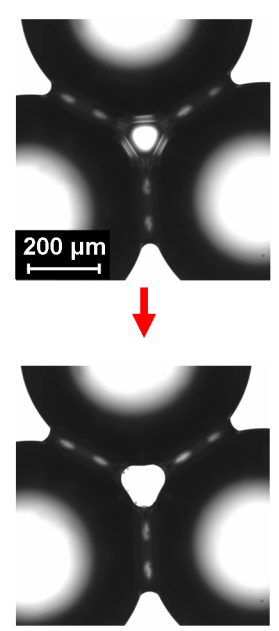

A trimer break into three capillary bridges. c)

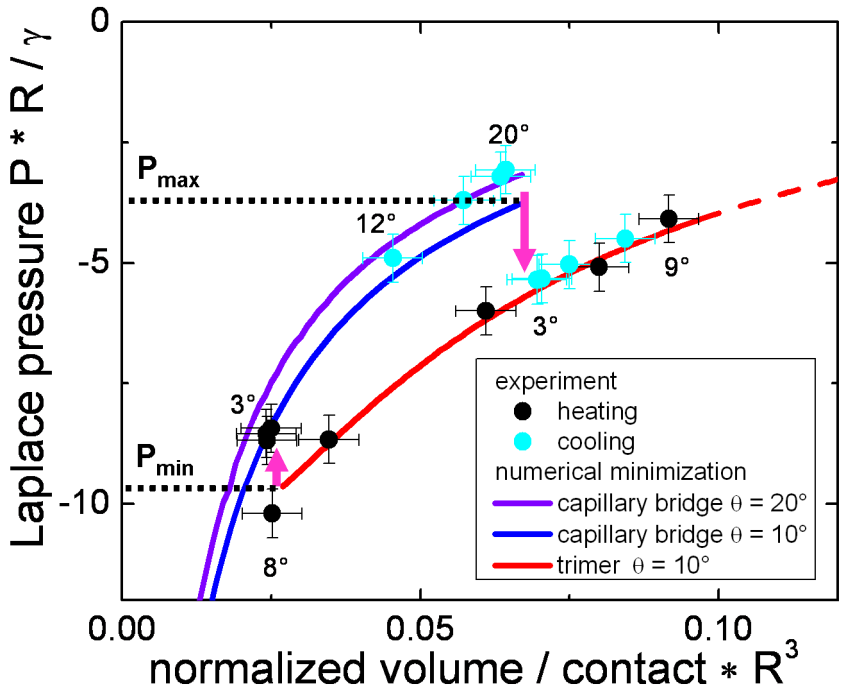

Figure 4.10: a) Schematic of a trimer (top) which breaks into three capillary bridges (bottom) b) Optical microscope images of an evaporating trimer which breaks at the critical Laplace $P_{\min }$ pressure into three capillary bridges. c) Laplace pressure of trimers and capillary bridges as a function of their volume normalized by the bead radius and the number of contacts. An evaporating trimer (black dots) decays into three capillary bridges. The condensing capillary bridges (blue dots) merge into a trimer. The experimental data is compared to lines derived by numerical minimization [54, 60] of capillary bridges $\left(\theta=10^{\circ}\right.$ blue line, $\theta=20^{\circ}$ pink line $)$ and trimer $\left(\theta=10^{\circ}\right.$ red line $)$.

Figure 4.10 b) shows optical microscope images of an evaporating trimer between ruby beads with radii $R=300 \mu \mathrm{m}$. At the critical minimal Laplace pressure $P_{\min }$ the trimer decays into three capillary bridges. A complete transition loop between trimers and capillary bridges, i.e. formation and breaking, is shown in Figure 4.10 c) [54, 60]. The volume is normalized by the bead radius and by the number of contacts in order to allow a comparison of the results for different liquid clusters and capillary bridges. The experiment was started with three capillary bridges between three ruby beads in contact. By use of a peltier cooling device, the temperature was lowered and the capillary bridges' volume increased by condensing water from the gas phase as shown by the blue dots in $4.10 \mathrm{c}$ ). As the capillary bridges reached a volume per contact $V / C$ of 0.067 corresponding to the normalized critical Laplace pressure 
$P_{\max }=-4.5$ the capillary bridges merged into a trimer and the normalized LP dropped to a value of -5.7. With a further increase of the volume the Laplace pressure also increased. Then the sample was heated and the volume of the large trimer decreased as shown by the black dots in Figure 4.10 c) and the Laplace pressure dropped. At a normalized volume per contact $V / C$ of 0.027 and a normalized LP of $P_{\text {min }}=-9.9$ the trimer decayed into three capillary bridges with a normalized LP of -7.8. As the liquid volume is decreased the Laplace pressure decreases further. The blue line $\left(\theta=10^{\circ}\right)$ and the pink line $\left(\theta=20^{\circ}\right)$ in Figure 4.10 c) display the normalized Laplace pressure of a capillary bridge and the normalized LP of a trimer $\left(\theta=10^{\circ}\right)$ as a function of the normalized liquid volume derived by numerical minimization [54, 60]. In this measurement we have captured the complete transition loop from a trimer to capillary bridges and back into a trimer.

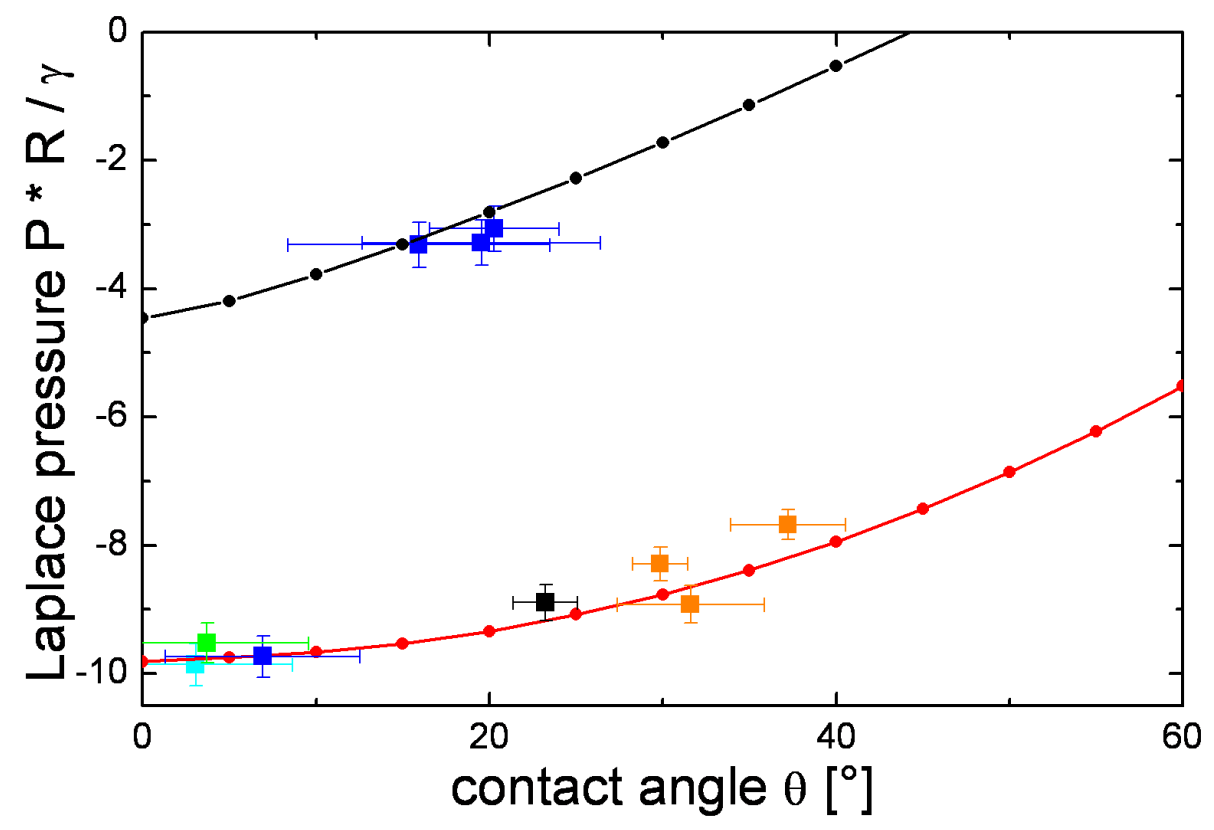

Figure 4.11: The critical normalized Laplace pressure $P_{\min }$ and $P_{\max }$ for the instability of a trimer, respectively three capillary bridges as a function of the contact angle $\theta$. As wetting liquids, water (light blue square), ethylene glycol (black square), diiodomehtane (orange square), $\mathrm{ZnI}_{2}+$ surfactant (blue square) and, silicone oil (green square) were used. The experimental data is compared to the values of $P_{\min }$ and $P_{\max }$ obtained by numerical minimizations [54, 60].

Figure 4.11 displays the critical minimal and maximal normalized LP, i.e. where the trimer, respectively, three capillary bridges become unstable for various liquids as a function of the contact angle. Here, we used water (light blue squares), $\mathrm{ZnI}_{2}$ salt solution with added 
dishwasher surfactant (blue square), silicon oil (green square), ethylene glycol (black square), diiodomethane (orange square) as wetting liquids in order to vary the contact angle on the ruby beads. We found that both critical Laplace pressures $P_{\min }$ and $P_{\max }$ increase with increasing contact angle. We compared our experimental results with numerical minimizations [54, 60] and found good agreement within the errors of the measurements. It turned out to be difficult to measure small normalized Laplace pressures larger than -2 because the shape of the trimer becomes irregular due to pinning effects.

\subsubsection{Transitions between trimers and capillary bridges in a disordered triangular bead geometry}

Even for perfectly monodisperse beads we will find triangular bead configurations in a random pile which exhibit a small separation distance between two of the three beads. In the following, we will demonstrate that the transition between a trimer and three capillary bridges in such a typical bead geometry with an opening angle $\alpha>30^{\circ}$ differs significantly from the transition in an ideal configuration of three beads. To explore the influence of the opening angle we employed the measurement setup described in the previous section. We focused on evaporating the wetting liquid to measure the critical minimal normalized Laplace pressure $P_{\min }$ corresponding to the decay of a trimer. Optical microscopy images of a trimer in a triangular bead configuration with an opening angle $\alpha>30^{\circ}$ are shown in Figure 4.12 a) and b). As expected the trimer decays into three capillary bridges for opening angles close to an 'ideal' triangular bead configuration. If the opening angle is larger than approximately $32^{\circ}$ the trimer will decay into only two capillary bridges as shown in Figure $4.12 \mathrm{~b}$ ).

The experimentally measured curvatures of the liquid / air interface were used to calculate the normalized Laplace pressure shortly before the trimer becomes instable. The experimental obtained values are shown in Figure $4.12 \mathrm{c}$ ) and compared to corresponding results of numerical minimizations [54, 60]. We found three possible scenarios in which a trimer transforms into capillary bridges. The trimer can break up into three capillary bridges in a similar way as an ideal trimer at $\alpha=30^{\circ}$ (red dots), or with a flip into two capillary bridges (blue dots), or without a flip into two capillary bridges (green dots). Besides the liquid volume the opening angle $\alpha$ between the beads acts as a control parameter and defines the type of instability. Below a critical opening angle $\alpha<31.95^{\circ} \pm 0.05^{\circ}$ the trimer decays into three capillary 

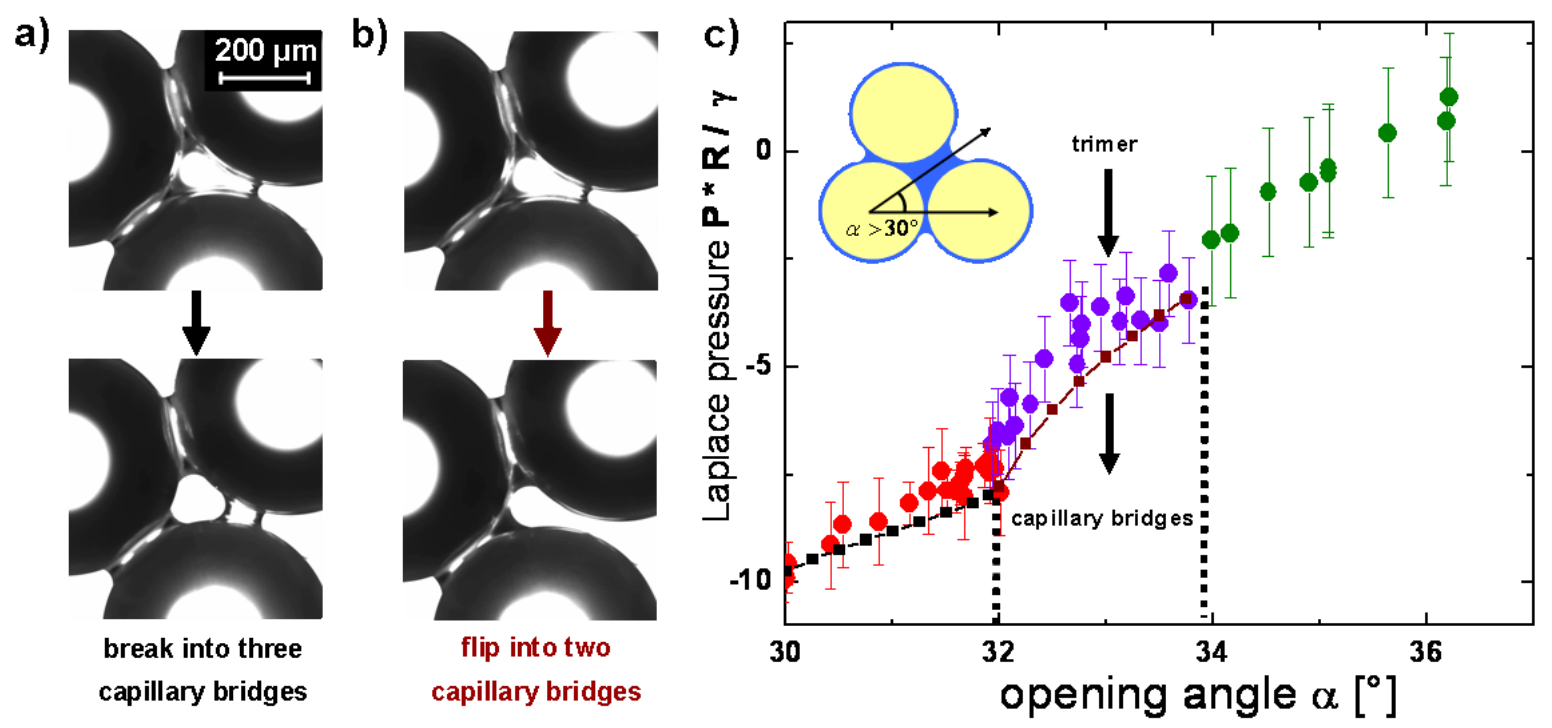

Figure 4.12: a) Optical microscopy images of a trimer with an opening angle $\alpha<32^{\circ}$. The trimer decays into three capillary bridges. b) At larger opening angles $\alpha>32^{\circ}$, only a flip into two capillary bridges occurs. c) Critical minimal normalized Laplace pressure $P_{\text {min }}$ of a trimer as a function of the opening angle $\alpha$. One can distinguish three different regimes: break up into three capillary bridges (red dots) between $\alpha=30^{\circ}$ and $\alpha=32^{\circ}$, a flip of the contact line and a subsequent break up into two capillary bridges (pink dots) between $\alpha=32^{\circ}$ and $\alpha=34^{\circ}$, and a slow transformation of a trimer into two capillary bridges at $\alpha>34^{\circ}$ (green dots). The solid lines are derived by numerical minimization [54, 60]. Inset: Definition of the opening angle.

bridges but the minimal normalized LP $P_{\min }$ increase with increasing $\alpha$. At an opening angle of $\alpha=31.95^{\circ}$ the minimal normalized LP $P_{\text {min }}$ reaches a value of -7.8. At $\alpha=31.95^{\circ}$ the type of instability changes. For opening angles larger than $31.95^{\circ}$ the trimer flips into two capillary bridges after the liquid meniscus has jumped into positions. At a further increase of $\alpha$ the jump becomes less pronounced and for opening angles above $\alpha>33.9^{\circ} \pm 0.1^{\circ}$ this discontinuous jump turns into a gradual motion.

In Figure $4.12 \mathrm{c}$ ) the experimental findings are compared with values of the normalized LP at trimer instability obtained from numerical minimization [54, 60]. As already found in our experiments, two regimes of instability can be distinguished, one corresponding to trimers decaying into three capillary bridges (black squares) and another corresponding to a decay into two capillary bridges (brown squares). The normalized LP at the numerically determined points of instability are slightly smaller than the values measured in experiment. This might be due to a contact angle of the wetting liquid being not exactly zero. 
Limited by the value of $P_{\min }$ the magnitudes of the Laplace pressure of trimers found in a random bead pile are smaller as compared to the LP of ideal trimers. Hence, the attractive capillary forces in such a pile will be, on average, weaker than in a regular dense packing of spherical beads.

\subsubsection{Transitions of larger clusters}

More complex liquid clusters such as pentamers, heptamers or tetrahedral cluster may undergo a variety of possible instabilities. Starting out from the fundamental units of a liquid cluster, i.e. the trimer and a filled tetrahedral gap we will illustrate the decay into smaller clusters and capillary bridges and the coalescence into larger liquid morphologies at a growing liquid volume. For these measurement we used an optical microscopy setup in transmission mode.

Since we are interested in liquid morphologies as they appear inside a random pile the liquid must not wet the cover slide. Evaporation and condensation of the wetting liquid was slow compared with the typical time scale of the hydrodynamic equilibration inside the cluster.

\section{Pentamer}
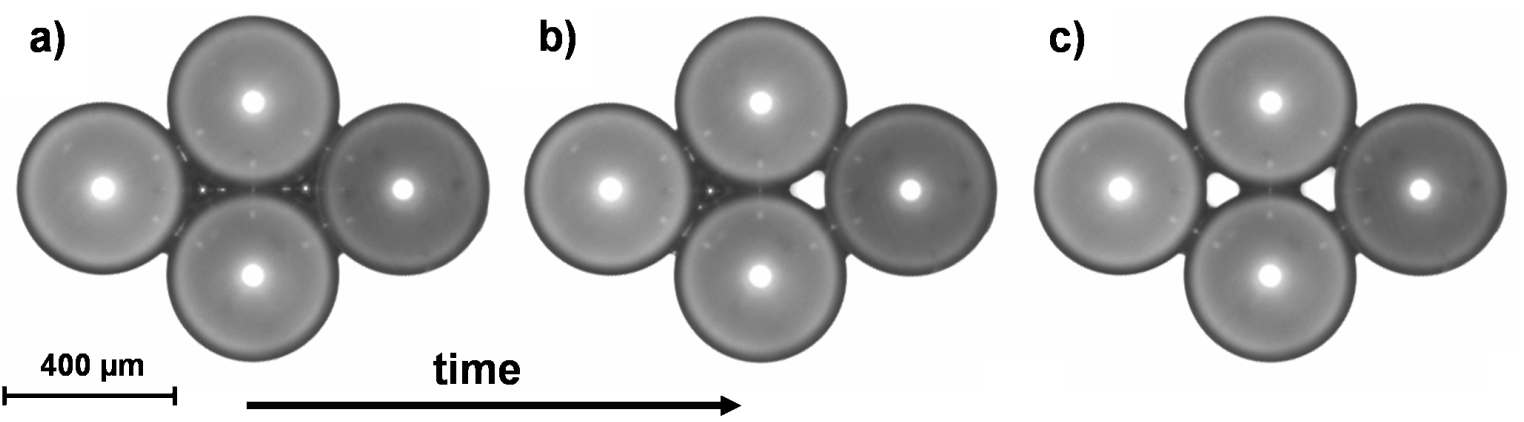

Figure 4.13: Optical microscopy images of a) an evaporating pentamer which breaks up into b) a trimer and later into c) five capillary bridges.

Figure 4.13 displays optical microscopy images of a pentamer consisting out of two trimer units. After decreasing the liquid volume the pentamer shown in Figure 4.13 a) breaks up into one trimer unit and two capillary bridges as illustrated in Figure $4.13 \mathrm{~b}$ ). These liquid morphologies are mechanically stable for the actual liquid volume. Further reduction of the liquid volume causes the trimer to rupture into three capillary bridges, cf. Figure $4.13 \mathrm{c}$ ) and 
the previous section. After full equilibration the Laplace pressure will be the same in all five capillary bridges.

The time delay between the first and second instability can be explained from a transport of volume from the trimer unit which breaks up to the remaining trimer unit. Due the fast equilibration process inside the remaining trimer, the Laplace pressure increases slightly which has a stabilizing effect.

The inverse process at increasing the liquid volume, i.e. the formation of a pentamer from five capillary bridges involves a coalescence of a trimer with two neighboring capillary bridges.

\section{Heptamer}
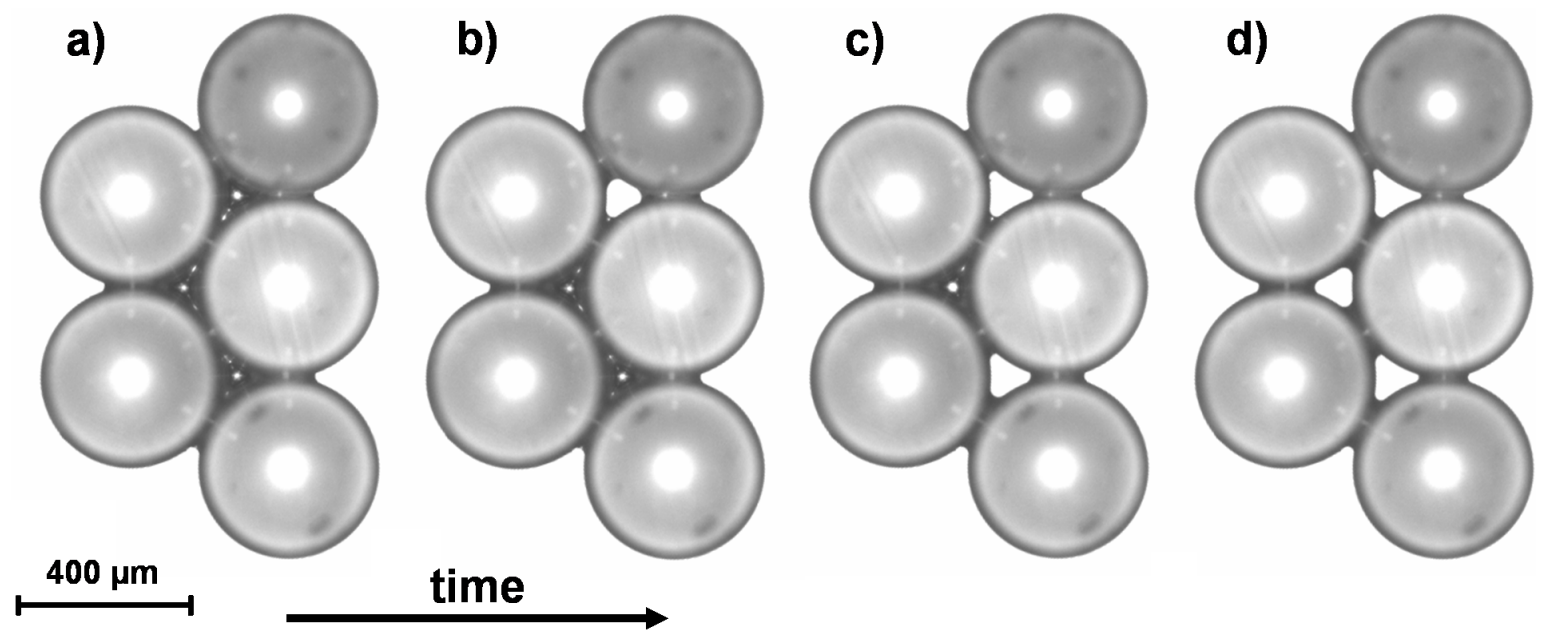

Figure 4.14: Optical microscopy images of an evaporating heptamer a) which breaks up into two capillary bridges and a pentamer b). The latter morphology breaks up into a trimer and two capillary bridges c). Final morphology consisting of seven capillary bridges d).

An liquid heptamer as shown in Figure 4.14 a) decays along two possible paths. In the first scenario the middle trimer unit breaks up into two trimers and a capillary bridge. In the second scenario a terminal trimer unit breaks up leaving behind a pentamer and two capillary bridges, cf. Figure 4.14 b). Upon a further reduction of liquid volume the pentamer decays into a trimer and two capillary bridges as shown in Figure $4.14 \mathrm{c}$ ). In the final state seven capillary bridges remain as illustrated in Figure 4.14 d).

For an increasing total liquid volume, the heptamer can be formed by merging two trimers with a capillary bridge or by merging two capillary bridges with a pentamer. 
One can easily see that the number of possible pathway grows with the cluster size, i.e. the number of capillary bridges. A large liquid cluster can be formed in many different paths. Each single transitions on this pathway are intrinsically hysteretic.

\section{Tetrahedral cluster}

We performed similar wetting experiments for a tetrahedral cluster. Judging from the bead geometry it is more challenging to image this non-planar structure by optical microscopy. As illustrated in the schematic in Figure 4.15 a) we observe the system from the top [60]. In all experiments we observed two consecutive interfacial instabilities: in the first instability the tetrahedral cluster shown in Figure 4.15 b) decays into a trimer and three capillary bridges. The second instability can be identified with the break up of a trimer, cf. Figure $4.15 \mathrm{c})$. After the break up of the trimer light can pass through the top bead if the microscope is used in transmission mode.
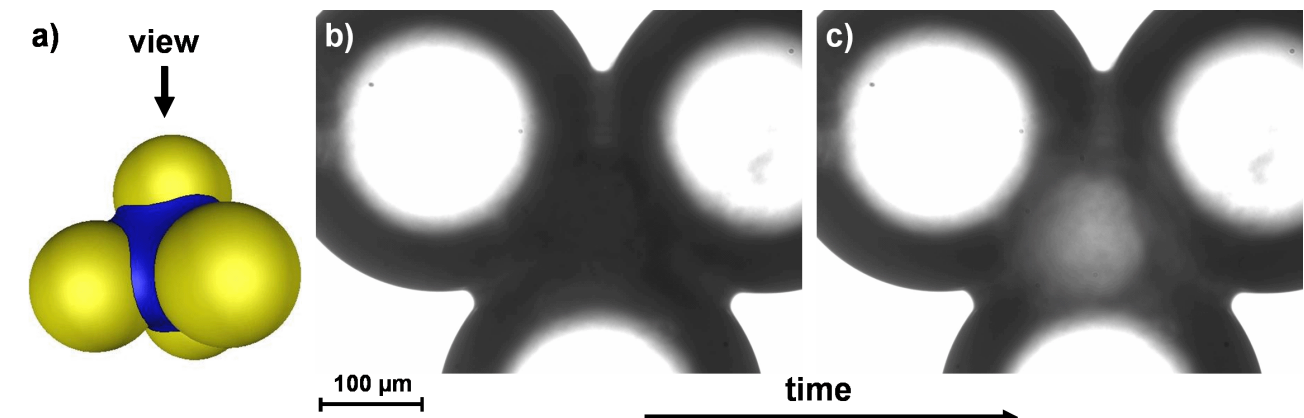

Figure 4.15: a) The arrow indicated the direction of view onto the tetrahedral cluster [60]. b) and c) show optical microscopy images of a tetrahedral cluster before instability. b) and after c) visible in the bright area in the center of the three visible beads.

Numerical minimizations of the interfacial energy in the corresponding tetrahedral bead geometry predict that the first instability occurs at a normalized Laplace pressure of -11 which is slightly smaller than the value at the instability of a trimer at -9.9. A tetrahedral cluster between equally sized beads in mutual contact breaks up into a trimer and three capillary bridges. However, it was not possible to reach the numerically predicted normalized LP at the instability in our experiments.

In this section we discussed transitions between small liquid morphologies, i.e. clusters involving a small number of capillary bridges. Optical microscopy is not able to capture transition between large liquid morphologies. 


\subsubsection{Forces of single clusters}

The force of capillary bridges and small clusters can be evaluated by numerical minimization of the interfacial energy using the Surface Evolver [61] as performed by Brinkmann [60]. In Figure 4.16 the attractive forces of a liquid wetting spherical beads of equal size in mutual contact are shown for a capillary bridge, a trimers, a pentamers, and a tetrahedral cluster as a function of the liquid volume at a fixed contact angle of $10^{\circ}$. The force is normalized by the maximal force of a capillary bridge between two beads in contact and the volume is normalized by the number of contacts and the bead radius. Only the axial component of the attractive force acting on a contact is shown. The force of capillary bridges between two beads in contact decrease monotonously with increasing volume. The normalized volume of a capillary bridge per contact is 0.065 at a half opening angle of $\beta=30^{\circ}$. At this point the capillary force is $20 \%$ smaller than the asymptotic value at small volumes. The trimer induces an almost volume independent force in the admissible range of volumes. The volume of the trimer is limited to small values by break up into capillary bridges on the one hand and to larger values by coalescence with neighboring capillary bridges on the other hand. As illustrated in the inset of Figure 4.16 two types of contacts corresponding to two different values of the attractive force can be distinguished in the pentamer [60]. The forces of the outer contacts (blue) is almost equal to the value found for a contact in a trimer (red, cf. inset in Figure 4.16). The central contact (green) of a pentamer exhibits a $10 \%$ larger force than the maximal capillary bridge force. The capillary force induced by a tetrahedral cluster is more sensitive to changes in the volume than in 'planar' geometries. We find a maximal normalized capillary force close to 1 at a normalized volume of approximately 0.055. For all liquid clusters the variation of the capillary force acting on a contact between two beads differs by only $20 \%$ from the maximal force due to the presence of a single capillary bridge.

Since we know that large liquid clusters are built out of trimer and tetrahedral units we can assume that the cohesive capillary forces in a wet granular pile does not change greatly for wetting liquids, even for larger clusters. The constancy of the Laplace pressure, the building principle of large clusters, and the volume insensitivity of the capillary forces explain the fact that the mechanical properties of wet granular piles remain constant over two decades of liquid content. 


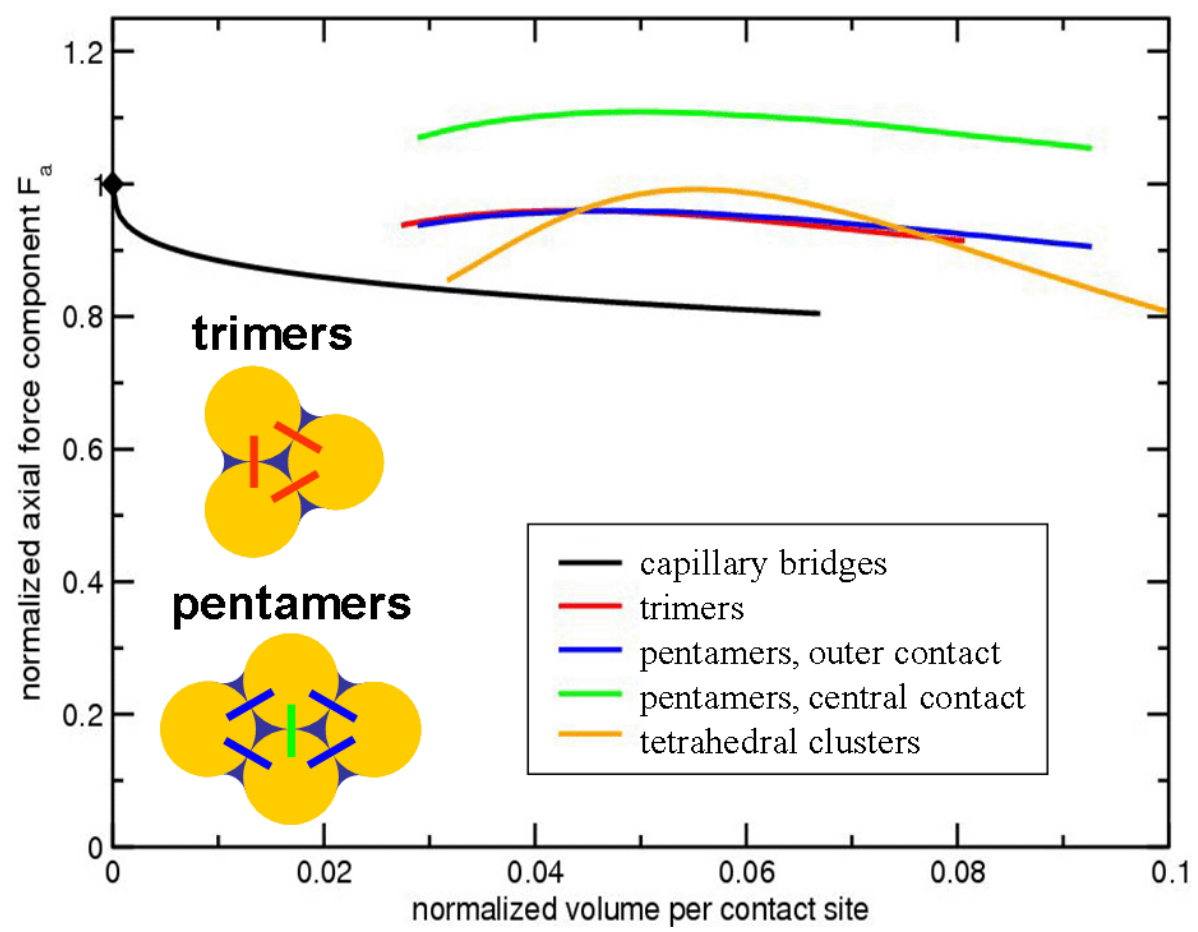

Figure 4.16: Capillary force acting on a contact as a function of the normalized volume per contact for different liquid morphologies [60]. The forces are divided by the maximal force of a capillary bridge The variation of all cluster forces differs only $20 \%$ from the maximal force of the capillary bridges.

\subsection{Large liquid clusters}

In the previous section we discussed the Laplace pressure and interfacial instabilities of capillary bridges and small liquid clusters. In experiments larger liquid cluster are difficult to observe with optical microscopy. We investigated larger liquid clusters at various liquid contents in random piles of glass beads using X-ray micro tomography. In experiments we employed aqueous $\mathrm{ZnI}_{2}$ solution as a wetting liquid and glass beads with a radius of $R=140 \mu \mathrm{m}$. With the image processing discussed in chapter 2 we sorted the liquid clusters according to their volume. In this way, we identified trimers, pentamers, heptamers, and tetrahedral clusters. One method to distinguish between different morphologies is to characterize them by their total surface area (liquid / air and liquid/glass interface) and volume. 


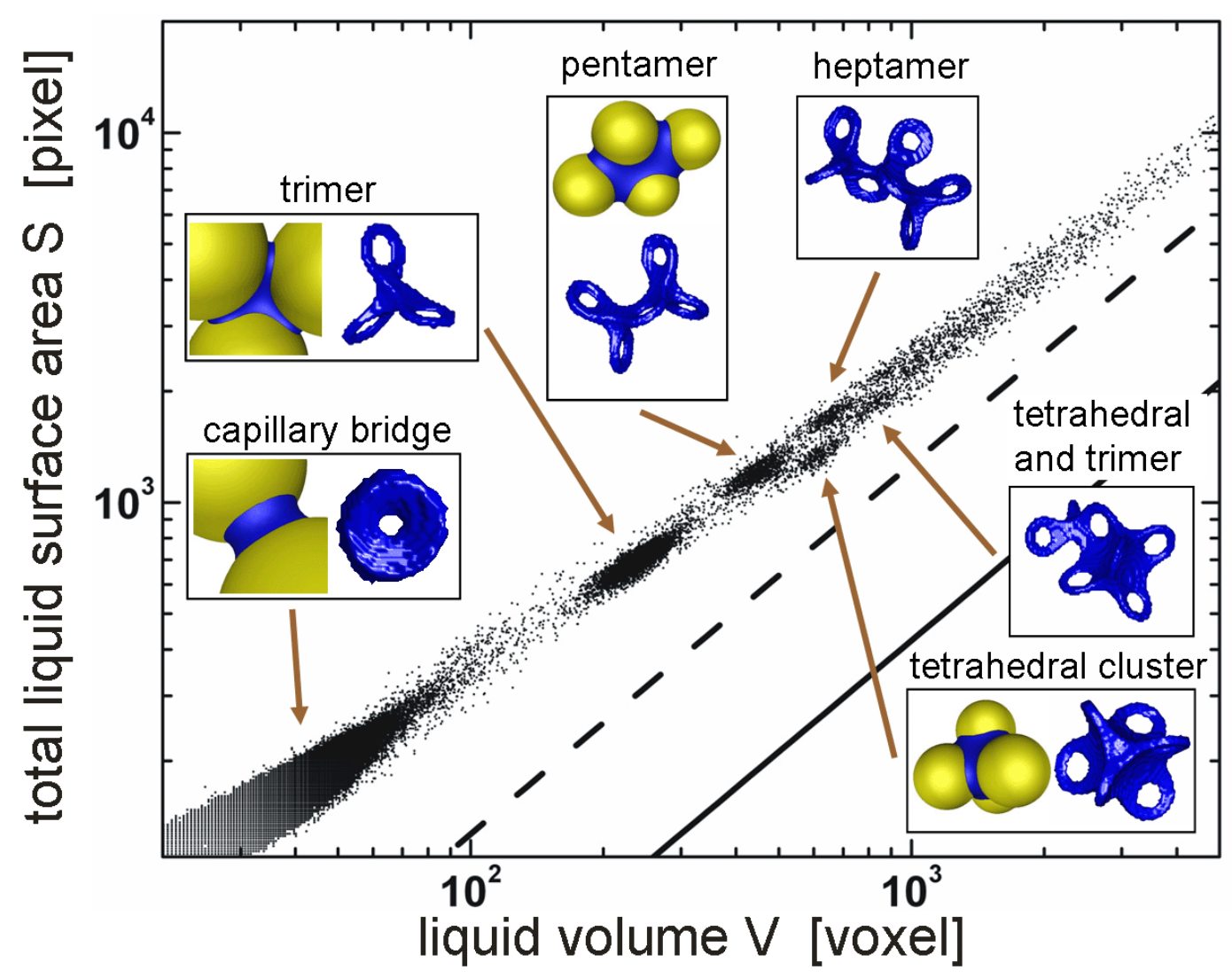

Figure 4.17: Surface area as a function of the liquid volume of individual liquid morphologies found by X-ray micro tomography in a random pile of glass beads with a radius of $R=140$ $\mu \mathrm{m}$ and at a liquid content $W=0.035$. Each data point in one of the clouds corresponds to a liquid cluster or capillary bridge [79]. The insets show examples of these morphologies in comparison to numerical minimization of the interfacial energy [60]. As indicated by the dashed line the clusters show a linear scaling between the total liquid surface area and the liquid volume. The solid line is the area to volume scaling of a compact cluster. 
We extracted the volume and total surface area (liquid/grain surface plus liquid/air surface) of all capillary bridges and liquid clusters in our X-ray tomography images of samples with a liquid content $W=0.035$ and plotted them in Figure 4.17] [79]. In this plot certain accumulations of data points in the form of 'clouds' are visible which represent a specific liquid morphology such as a capillary bridge, a trimer, a pentamer, a heptamer, a tetrahedral cluster, a tetrahedral cluster with a trimer, and larger clusters. The inset show examples of the morphologies corresponding to the clouds obtained by X-ray tomography and by numerical minimization [60]. All liquid clusters seem to follow a scaling curve becoming linear at sufficiently large volumes. In Figure $4.18 \mathrm{~b}$ ) the asymptotic linear scaling at large volumes, i.e. the surface area to volume ratio of a large cluster is indicated as a dashed line [62]. The solid line in Figure 4.17 [62] is the scaling of a compact liquid cluster, i.e. a cluster which completely fills the available pore space between the beads. The surface area to volume ratio of a compact cluster can be calculated for a pile of beads in a total sample volume $V_{0}$. The total surface area of the beads is $S=4 \pi N R^{2}$ where $\mathrm{N}$ is number of beads in the sample. The packing density of the beads is $\rho_{\text {packing }}=4 \pi N R^{3} / 3 V_{0}$ which is derived by the ratio of the volume occupied by the beads to the total sample volume. The volume of the liquid cluster liquid, $V$, equals the volume of the void space, and is given by $V=\left(1-\rho_{\text {packing }}\right) V_{0}$, where $\rho_{\text {packing }}$ is the packing fraction of the beads. Thus we find the ratio for the total surface area to the volume [62]:

$$
\frac{S_{\text {total }}}{V}=\frac{3 \rho_{\text {packing }}}{R\left(1-\rho_{\text {packing }}\right)} .
$$

The packing density of our grains is $\rho_{\text {packing }}=0.57 \pm 0.01$ as derived in chapter 2 .

If we compare the data clouds in Figure 4.17 with the solid line corresponding to compact liquid clusters according to Eq. 4.3 the clouds are systematically shifted to larger values. This result is independent of the liquid content and was found for all samples considered ranging between $W=0.005$ and $W=0.17$.

A large percolated cluster at a liquid content of $W=0.11$ and a bead size of $780 \mu \mathrm{m}$ is shown in Figure 4.18 a) [62]. The liquid content is in agreement with the percolation threshold found in Scheel [94]. An visual inspection of the cluster in Figure 4.18 a) reveals that the large cluster is constructed of trimer and tetrahedral cluster units. Increasing the liquid content of the bead pile will cause filling of triangular openings or tetrahedral gaps, leading to a constant surface to volume ratio of large clusters and a branched open structure. 
a)
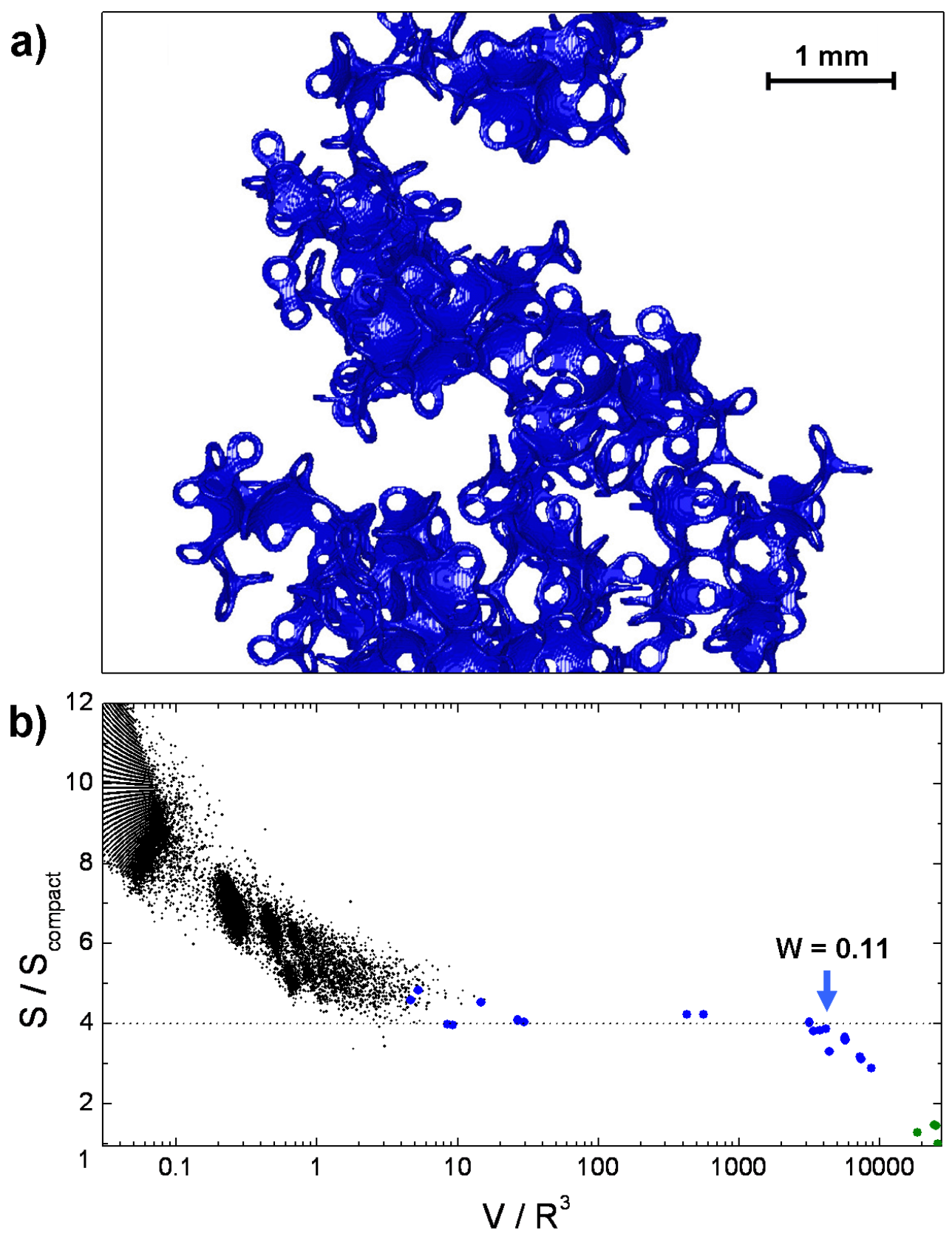

Figure 4.18: a) A large 3D rendering of a percolating liquid cluster as found by X-ray micro tomography at a liquid content of $W=0.11$ and bead size of $780 \mu \mathrm{m}$ [62]. b) Surface area to liquid volume ratio of all liquid clusters and capillary bridges in the sample (black dots) normalized by the surface area to volume ratio of a compact cluster at the same volume, cf. Eq. 4.3. The largest clusters found in different samples (blue dots) approach the dotted line indicating a surface to volume ratio of four, cf. also the dashed line in Figure 4.17. Only for very large percolating clusters found at liquid contents above $W>0.11$ the surface area to volume ratio approaches the value corresponding to compact filling (green dots). 
To visualize the excess of surface area of an open liquid cluster over a compact cluster we normalized the data in Figure 4.17 b) by Eq. 4.3 , i.e. we divided the experimentally determined surface area of a liquid cluster by the expected surface area of a compact cluster of the same volume. The cluster and capillary bridges (black dots) are plotted in Figure $4.18 \mathrm{~b}$ ) as a function of the normalized volume at a liquid content $W=0.035$ together with the normalized surface area to volume ratio of the largest clusters in several samples for liquid contents ranging from $W=0.01$ to $W=0.17$ (blue dots). Completely filling (green dots) corresponds to a normalized value of 1 . The capillary bridges and clusters are visible as clouds with a normalized value of approx. 9 for capillary bridges and approx. 7 for trimers. Large clusters approach a value of 4 (dotted line) and are found always remote from surface area to volume ratio for complete filling. At liquid contents of $W>0.11$ which is close to the estimated percolation threshold at $W=0.12 \pm 0.02$ [94], the largest cluster transforms into more compact structure. The largest clusters found in the sample exhibit a three times larger surface area then compact clusters. The green dots corresponds to a completely submerged sample, i.e. at the maximal liquid content.

For comparison we added the numerically obtained surface area to volume ratio of capillary bridges (blue line), trimers (red line), pentamers (green line), heptamers (rosa line), and tetrahedral clusters (orange line) to the experimental data at $W=0.035$, cf. Figure 4.19 . Each cluster curve is parameterized by the Laplace pressure between values $P_{\min }=-9.9$ and $P_{\max }=-4.5$, cf. Eq. 4.2. The end points of each curve match well with the surface area to volume ratio in the center of a cloud of the corresponding morphology at $P_{\max }$. This, again, is a strong evidence for the universal building principle of liquid clusters from trimer and tetrahedral clusters as basic units. 


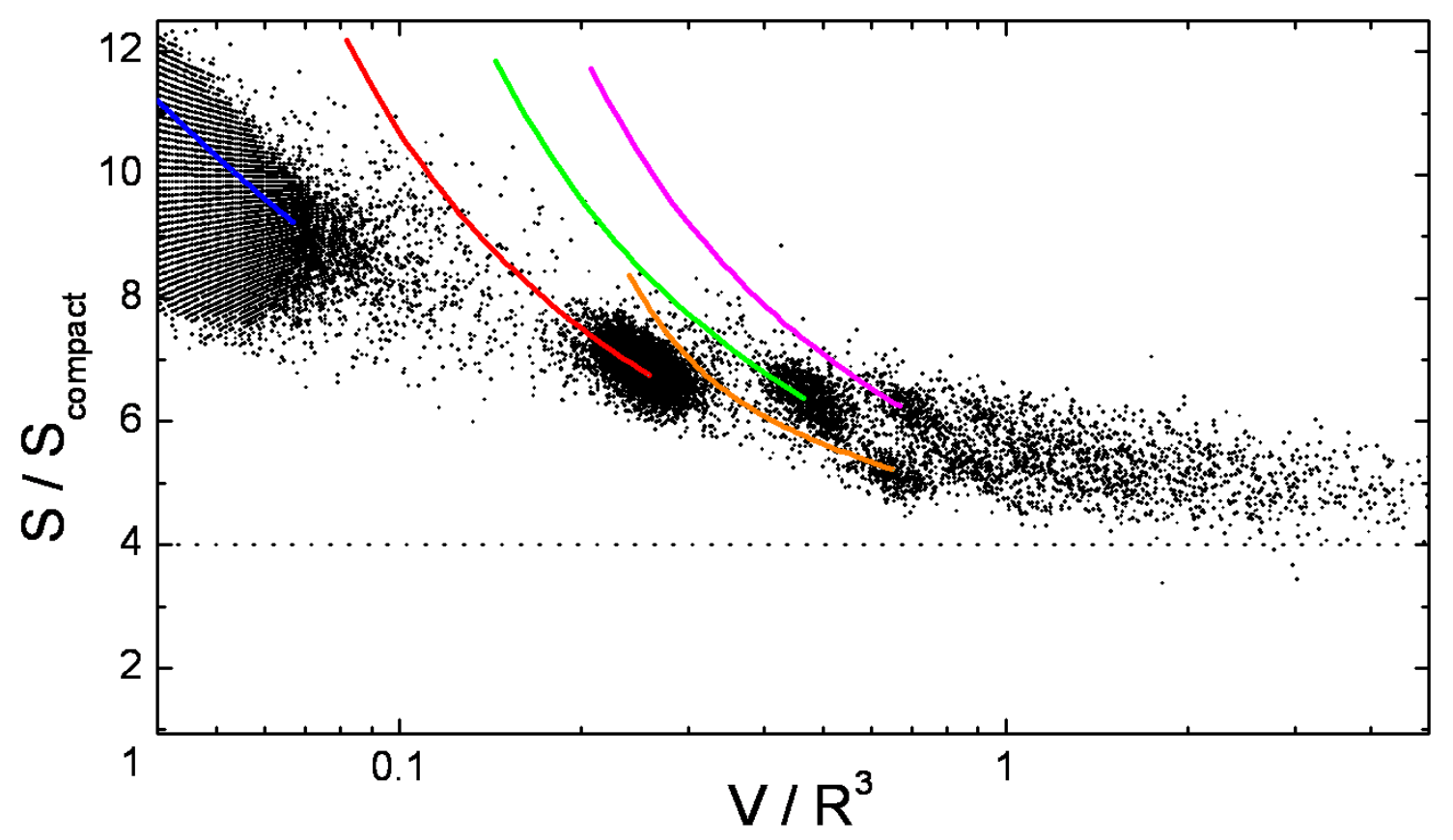

Figure 4.19: Surface over volume plot of the same data as in Figure 4.17 compared with predictions for capillary bridges (blue line), trimers (red line), pentamers (green line), heptamers (pink line), and tetrahedral clusters (orange line) obtained by numerical minimization [60]. The starting and end points of the curves are given by the critical Laplace pressure $P_{\text {min }}$, respectively, $P_{\max }$. The end points meet the corresponding clouds of the liquid morphologies. 


\subsection{Characterization of sand in comparison with glass beads}

The linear scaling of the surface area to volume ratio found in random piles of glass beads poses the question whether this results still holds for random packings of other irregularly shaped grain such as wet sand. In order to obtain samples with a comparable size we sieved sand (received from a glacial deposit near Marzahne, Brandenburg, Germany) to the same size fractions of $355-500 \mu \mathrm{m}$ as in previous experiments with glass beads. We measured the surface to volume ratio of the liquid clusters in exactly the same way as was done for the clusters in a pile of glass beads. The analyzed X-ray micro tomography images at a liquid content $W=0.03$ are shown in Figure 4.20 in comparison to glass beads of the same size [62]. The insets illustrate the clusters in a pile of glass beads and in wet sand. By inspection of Figure 4.20 the surface area to volume ratio for liquid clusters in a sand pile exhibits the same linear scaling behavior as for liquid clusters in a random pile of glass beads. The only difference is that there were no distinguishable clouds of liquid clusters. This can be easily explained by the polydispersity in shape and size of sand grains.

Mechanical measurements of the cohesive forces in wet sand show the same insensitivity with respect to the liquid content as was found in piles of wet glass beads. This agrees with the observation of open and branched liquid clusters in a wet sand pile. The building principle for liquid cluster in piles of spherical beads appears to be also valid in piles of sand grains. So far, we have used only liquids with a small contact angle on the surface of the grains. The influence of the wettability of the grains, i.e. the effect of the contact angle on the morphology of liquid clusters is not yet clear.

\subsection{Open versus compact morphology}

Most of the mechanical measurements and all X-ray micro tomographies of wet piles of glass beads, respectively, wet sand presented in the last section were done using a wetting liquid. In chapter 3 we have seen that the contact angle influences the capillary cohesion between the grain in a pile significantly. Optical microscopy imaging of a fluorescing liquid which forms a small contact angle on the surface of the beads, once again shows the existence of an open and branched liquid morphology in equilibrium, cf. Figure 4.21 a). At large contact angles $\left(\theta=45^{\circ}\right)$, e.g. for water in a pile of PMMA beads, more compact liquid morphologies 

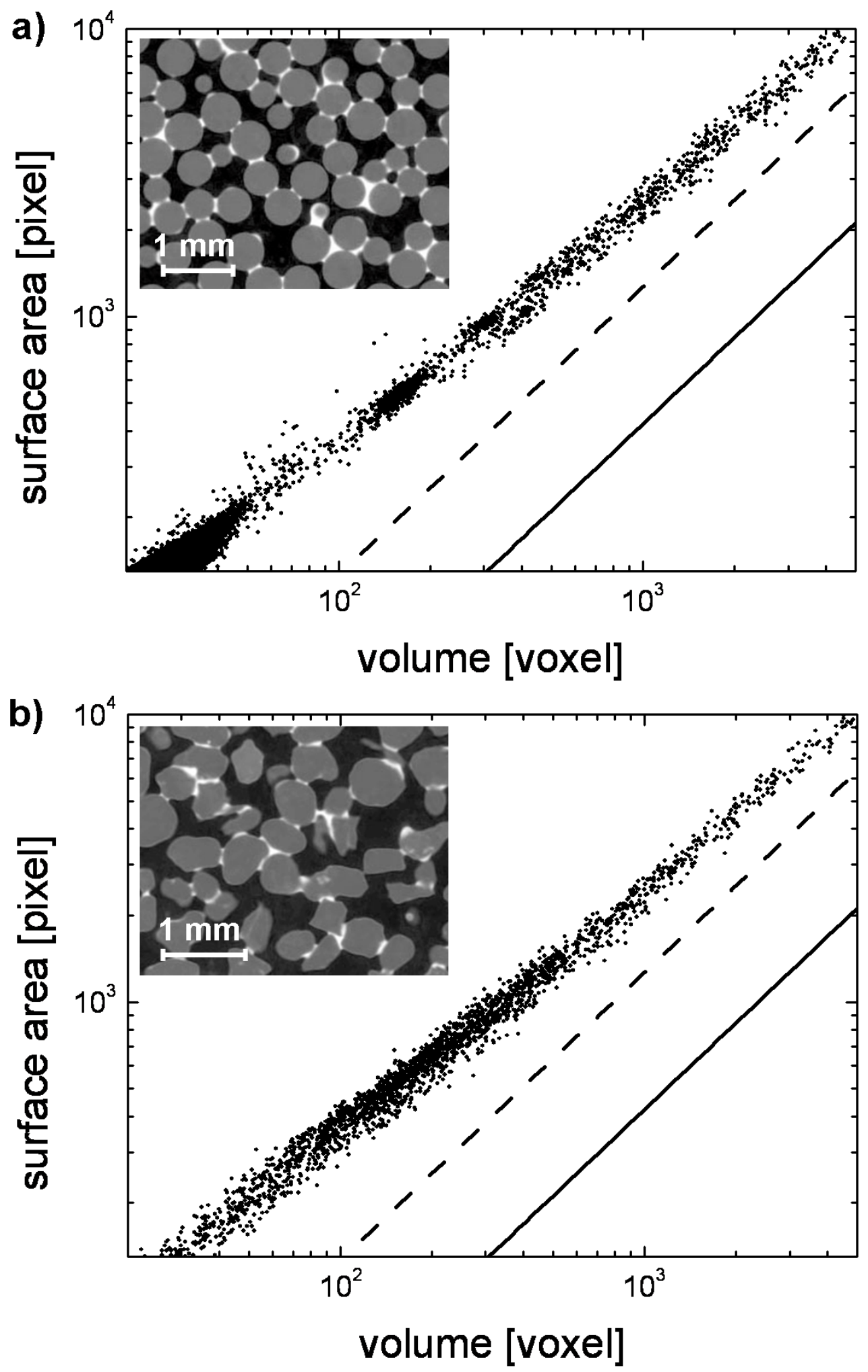

Figure 4.20: Comparison of the surface area to volume ratio of liquid morphologies in a) a random pile of glass beads and in $b$ ) a sample of wet sand [62] for a liquid content of $W=0.03$. The insets show a slice through X-ray micro tomography images of the samples. 
are formed as shown in Figure $4.21 \mathrm{~b}$ ).

In addition to the contact angle, the liquid content and packing density of the beads control the formation of certain liquid morphologies. Obviously, higher packing densities will force the liquid into a compact morphology already at lower liquid contents. Even for low contact angles, compact clusters are found at liquid contents sufficiently close to saturation.

We performed experiments with a variety of bead materials with different contact angles (glass beads, PMMA beads, ruby beads, diamonds, hydrophobic sand, basalt beads) and at different packing densities. The results are summarized in Figure 4.21 c). Here, open symbols correspond to open morphologies while closed symbols to compact morphologies. Figure 4.21 c) illustrates the experimentally found types of liquid morphologies in comparison to theoretical predictions in the plane of packing densities and contact angles. For wetting liquids with zero contact angle on glass beads we did not find any compact structure in the whole range of available packing densities. At contact angles larger than approximately $50^{\circ}$, however, we found compact liquid morphologies in all samples.

The boundaries in the plane of packing densities and contact angles between regions of open and compact morphologies can be calculated from a simple model [60]. According to this model, one may distinguish two main cases: In the first case, a network of trimers (open morphology) represents the minimum of interfacial energy in a certain intermediate range of liquid contents. In the second case, either capillary bridges or compact morphologies represent the stable phase, leaving out the network of trimers as a global energy minimum. In a certain range, the liquid in the pile may be found in one or the other state depending on the history of preparation. The red line in Figure $4.21 \mathrm{c}$ ) is the limit above which the trimer phase is missing [60]. The blue line in Figure 4.21 c) delimits the region where definitely the trimer network appears as a minimum of the interfacial energy in a certain range of liquid contents [60]. Between both lines open and compact liquid morphologies can be found.

Our experimental data are consistent with the regions assigned to certain liquid morphologies, as predicted by the model calculations. Below the blue line only open morphologies have been observed. In the region between both lines we found compact and open morphologies. Above the red line exclusively compact morphologies appeared. 


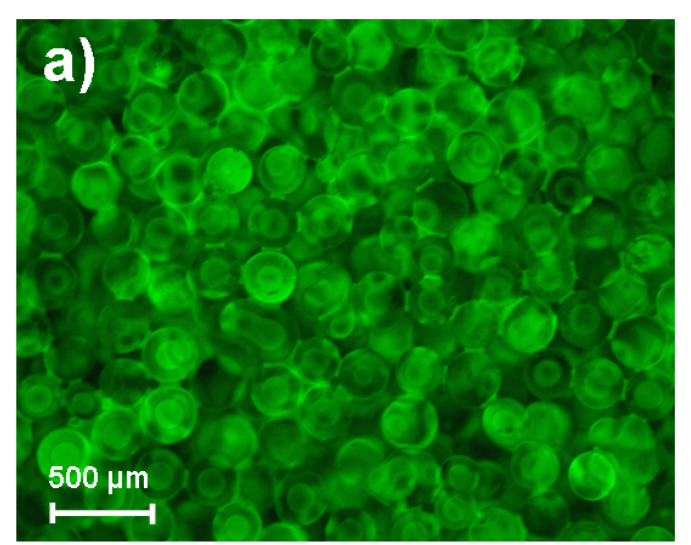

open morphology, $\theta=0^{\circ}$

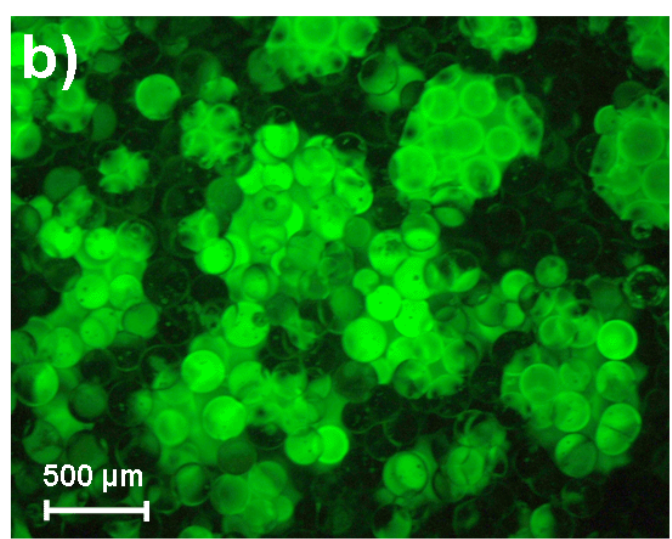

compact morphology, $\theta=45^{\circ}$

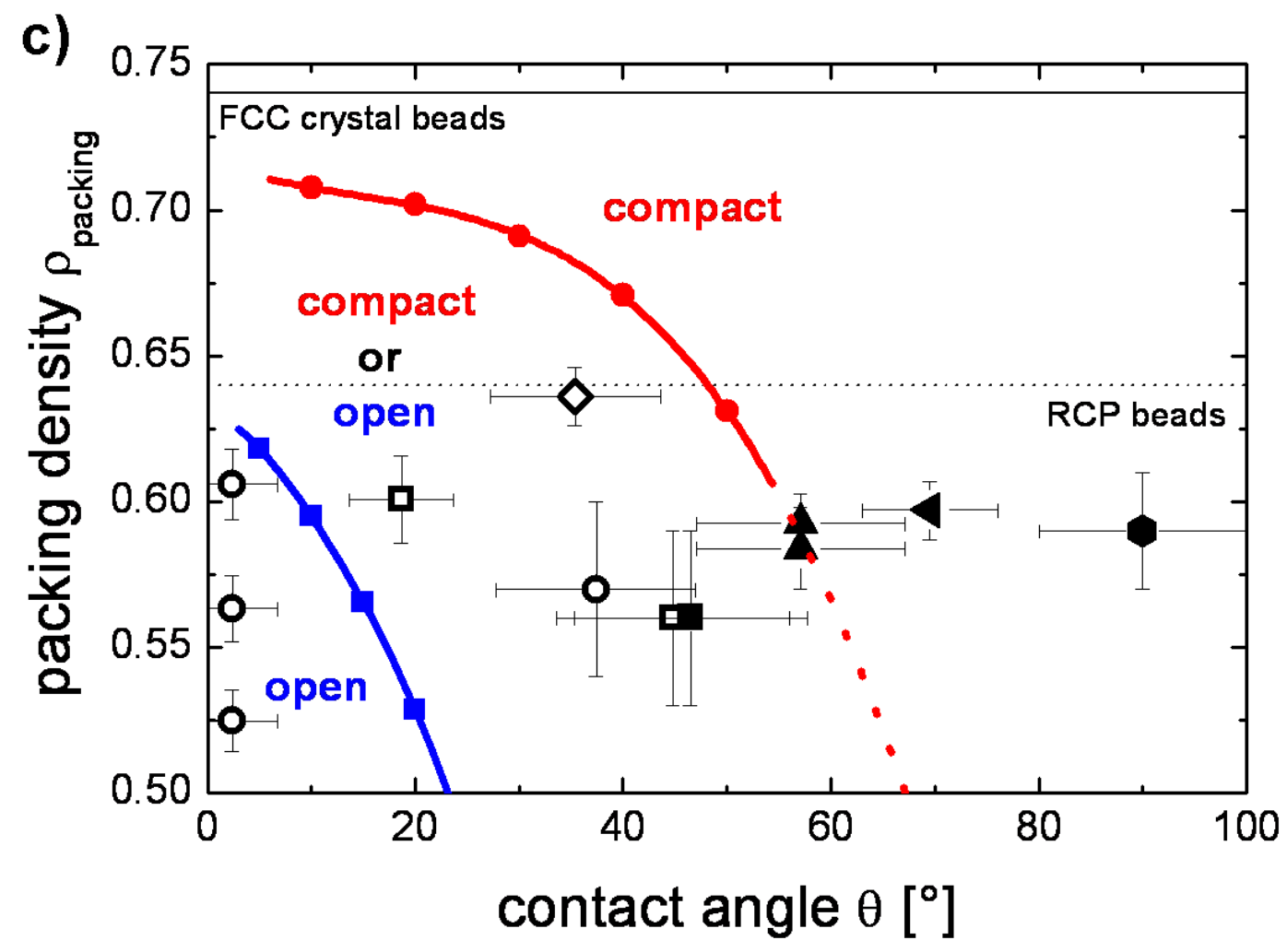

Figure 4.21: a) Optical fluorescence microscopy image of a fluorescence liquid in a pile of glass beads with a small contact angle $\theta=0^{\circ}$ and shows an open liquid morphologies whereas b) shows liquid in a pile of PMMA beads at a large contact angle $\theta=45^{\circ}$ shows a compact liquid morphologies. c) Morphological diagram for open and compact liquid morphologies as a function of packing density and contact angle. The used beads are glass beads (round symbols), PMMA beads (square), ruby beads (triangle), diamonds (diamonds), hydrophobic sand (pentamer), and basalt beads (left pointing triangle). Open symbol means that the liquid forms an open morphology and closed symbol a compact morphology. The red line is the limit above only compact morphologies are formed [60] and the blue line is an upper limit where only open morphologies are formed [60]. The black solid line is for a FCC bead crystal and the dotted line for a RCP of beads. 


\section{Chapter 5}

\section{Summary and Outlook}

\subsection{Summary}

The constancy of the mechanical properties of a wet granulate over about two decades of liquid content $W$ (liquid volume / total volume) for the wetting case was observed by three mechanical experiments. We used a shaker for the vertical critical fluidization acceleration, a shear cell for the shear stiffness, and a centrifuge for the tensile strength. Employing X-ray micro tomography we explored the liquid morphologies up to the completely submerged case. We found the trimer as the liquid cluster with the highest frequency. Larger liquid clusters are composed of connected trimers and tetrahedral clusters as basic units. For wetting liquids these large clusters exhibit an open morphology provided the liquid content in the sample is not too close to saturation. We observed an equilibration process driven by Laplace pressure between the capillary bridges and clusters. For the particular aqueous salt solution we used as a wetting liquid the volume transport proceeds via a liquid film on the surface of the grain. The Laplace pressure after equilibration is independent on the liquid content $W$ between 0.025 and 0.20 since clusters with a Laplace pressure larger than a critical value will coalesce with surrounding clusters or capillary bridges. The constancy of the Laplace pressure together with the formation of open liquid morphologies causes the insensitivity of the mechanical properties of a wet granulate with respect to the liquid content. At large liquid contents close to the fully submerged case the Laplace pressure rises and the clusters become more compact; at the same time the stiffness of the material is reduced.

For a partial-wetting configuration like water on basalt beads with a large contact angle $\theta=70^{\circ}$ the mechanical properties of wet grains differs significantly from the case of a wetting liquid. 
The lower stiffness in the critical fluidization acceleration could be explained by a decrease of the capillary forces with increasing contact angle. The larger stiffness measured with the shear cell, contradicting the expected reduction, could be mostly explained by contact angle hysteresis during stretching of a capillary bridge. The tensile strength measurements show the same effect at small liquid contents. The decrease of the tensile strength at $W>0.01$ could be explained by the decreased number of capillary bridges on a bead and the existence of compact clusters at large contact angle. The different liquid morphologies, open and compact, were explored by X-ray micro tomography and we found open and compact morphologies depending on the packing density and the contact angle.

To check the universality of our findings we investigated the effect of grain shape on the mechanical behavior and the liquid morphologies inside the pile. Measurements of the critical fluidization acceleration demonstrates that wet sand has the same mechanical behavior as a pile of glass beads at the same liquid content. Images of wet sand taken with X-ray microtomography reveal an open morphology of the wetting liquid and evidence that large clusters are again built out of small clusters as in the case of spherical beads. This allows us to state that our findings are also valid for non-spherical grains.

\subsection{Outlook}

The largest part of my thesis is devoted to X-ray micro tomography. This technique allows us to answer many interesting questions. Four example projects will demonstrate the possibilities X-ray micro tomography offers. The first example project is the time resolved observation of propagating liquid fronts in a piece of sandstone, which could clarify how mineral oil can be pumped out of the rock. The second project is a flight path measurement of tracer beads in a fluidized granular bed, giving access to parameters like diffusion and granular temperature. The objective of the third project is imaging of liquid morphologies forming at contact points in a network of cylindrical fibers. The shape of the liquid at the contact points can be recorded by tomography and compared with corresponding morphologies derived by numerical minimization [60] using the software Surface Evolver [61]. The fourth and last project is the experimental measurement of packing densities of cylindrical segments of different aspect ratio. In a certain range of the aspect ratio, cylinders allow a higher packing density compared to a random close packing of spherical beads. 


\subsubsection{Liquid fronts in sandstone}

Liquid fronts are very important in science. One field of application is oil recovery, where oil is pressed out of the oil-bearing stone. In an oil field, not all oil can be pumped out of the stone. Normally, about $50 \%$ remains in the ground, depending on the type of stone.

Driven liquid fronts in sandstone can be visualized very well with X-ray micro tomography since a complete 3D tomography last less than one second if performed at beamline ID15A (ESRF, 2008). The first steps in this direction have already been done. We showed that driven oil and water fronts can be visualized using X-ray micro tomography. Figure 5.1 a) shows the dry sandstone whose pores are of about $200 \mu \mathrm{m}$ on size. Figure 5.1 b) was filled with an aqueous $\mathrm{ZnI}_{2}$ salt solution. All free pores are now filled with the liquid which appear bright in the image. At the left- and right hand sides of the image, there are many pores not filled with salt solution but with epoxy glue, to channel the liquid. Figure 5.1 c) shows the sample after oil was pumped through. Some of the pores are now dark due to the oil. The last image, Figure 5.1 d), shows the sample as the pumps were driven in reverse and the salt solution was pumped back. Some of the oil remains in the sample. All the images show how good a sample of oil and water could be visualized with X-ray micro tomography.
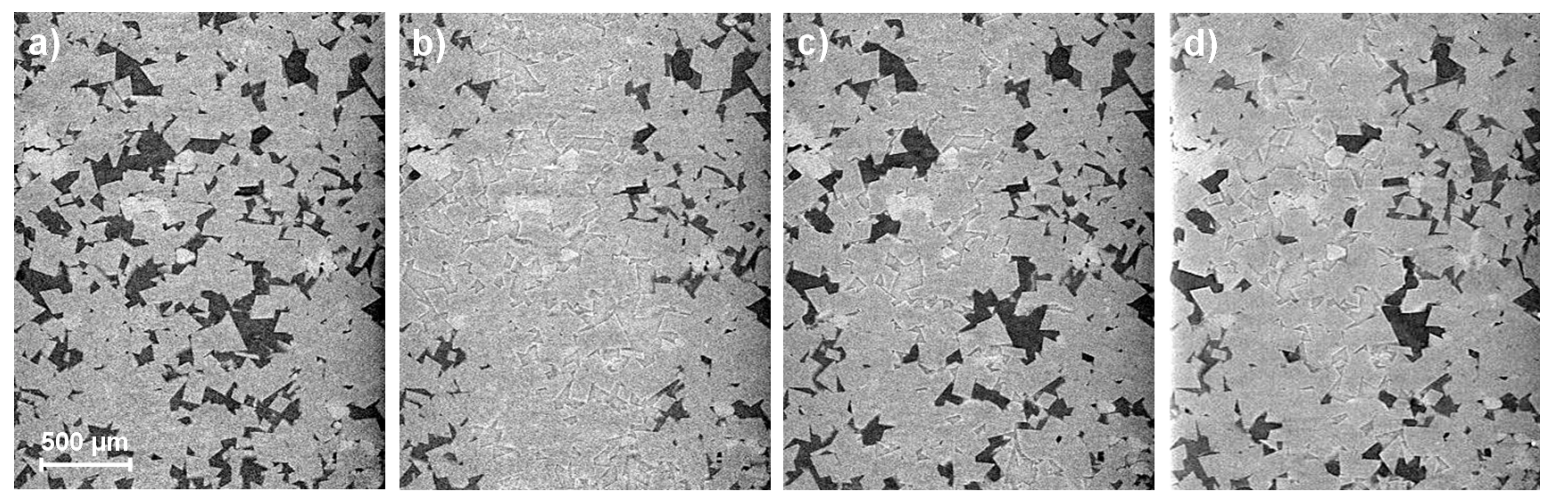

Figure 5.1: All images show a sandstone in the same vertical slice. a) The dry stone, b) filled with $\mathrm{ZnI}_{2}$ salt solution, c) after oil was pumped threw the sample c) after the salt solution was pumped back. 


\subsubsection{Flight path measurements}

Flight path measurements give access to parameters like diffusion, convection, and the granular temperature. Many two-dimensional experiments was done in the past but threedimensional experiments are very rare, and experiments with wet grains are even rarer.

The technique of our flight path measurement was developed during this thesis. The X-ray images were taken at the beamline ID15A (ESRF). We improved the measurements and optimized the choice of the tracer particles. The best tracer was a highly absorbing gold bead of radius $R=120 \mu \mathrm{m}$ with a lacquer covering. These tracer beads have nearly the same average density as glass beads, so buoyancy effects can be neglected. In Figure 5.2 ten lacquer-gold tracers are shown. The red line is the path of one tracer and shows a convection roll with diffusion and caging effects. The flight path measurement is a project of Zeina Khan at the MPI-DS Göttingen and Ralf Seemann at Saarland university.

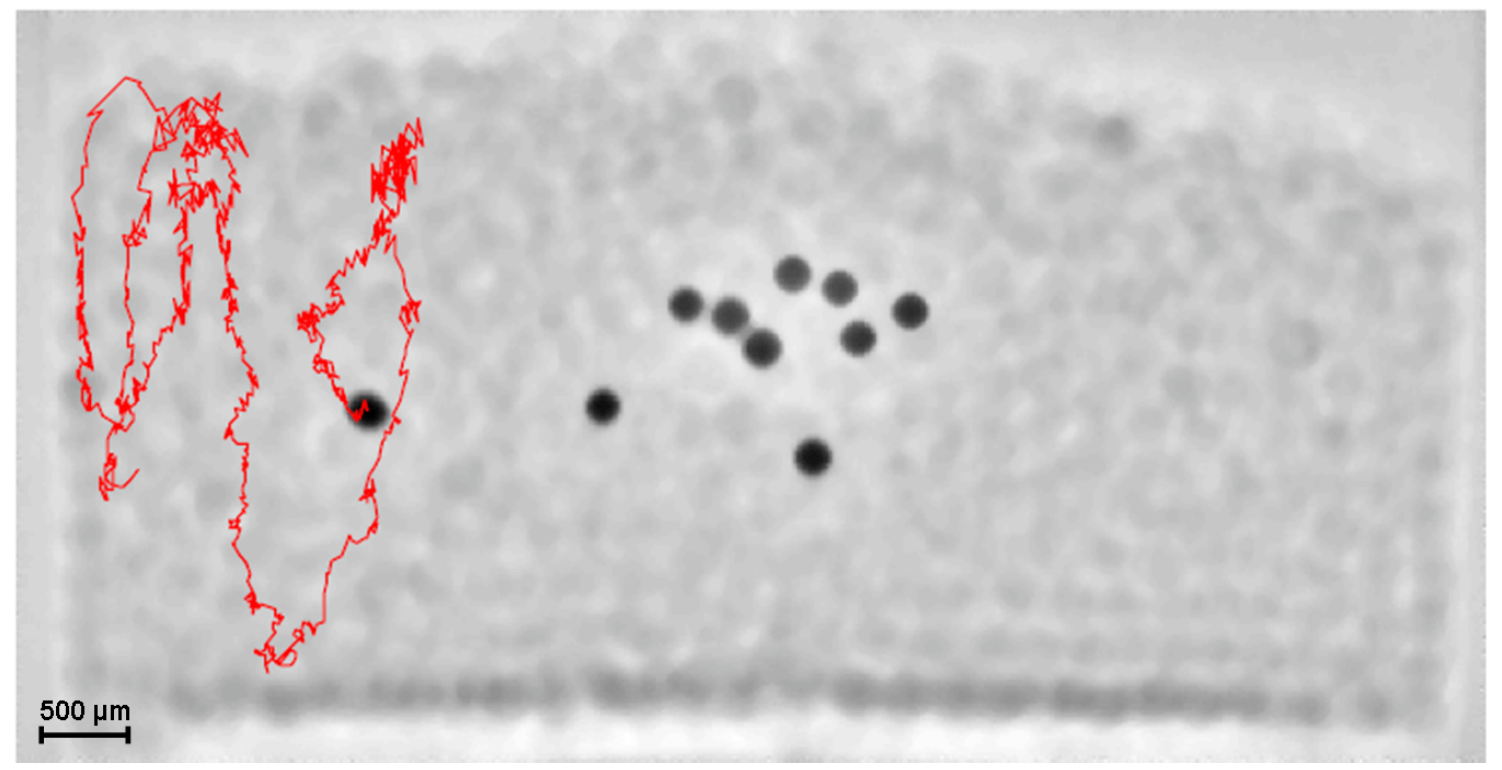

Figure 5.2: Ten gold tracer surrounded by a lacquer layer (black circles) are in fluidized glass bead sample with a bead radius of $170 \mu \mathrm{m}$. The position of one tracer during vertical agitation is shown as a red line. 


\subsubsection{Liquid clusters in a fiber network}

$\mathrm{X}$-ray micro tomography is an appropriate tool to study the formation of liquid clusters in a network of cylindrical glass fibers. The glass fibers shown in Figure 5.3 a) are $10 \mathrm{~mm}$ in length and with a radius of $150 \mu \mathrm{m}$. The fibers are loosely packed and the capillary forces of the liquid at $W=0.01$ are strong enough to rearrange the fibers into groups or stacks. Liquid clusters between fibers can be analyzed in the tomography images and compered with liquid shapes derived by numerical minimization [60] with the program Surface Evolver [61]. Figure 5.3 b) illustrates that liquid clusters between fibers can be in an elongated or localized, droplet-like state. This project is currently persuit by Martin Brinkmann at the the MPI-DS in Göttingen.
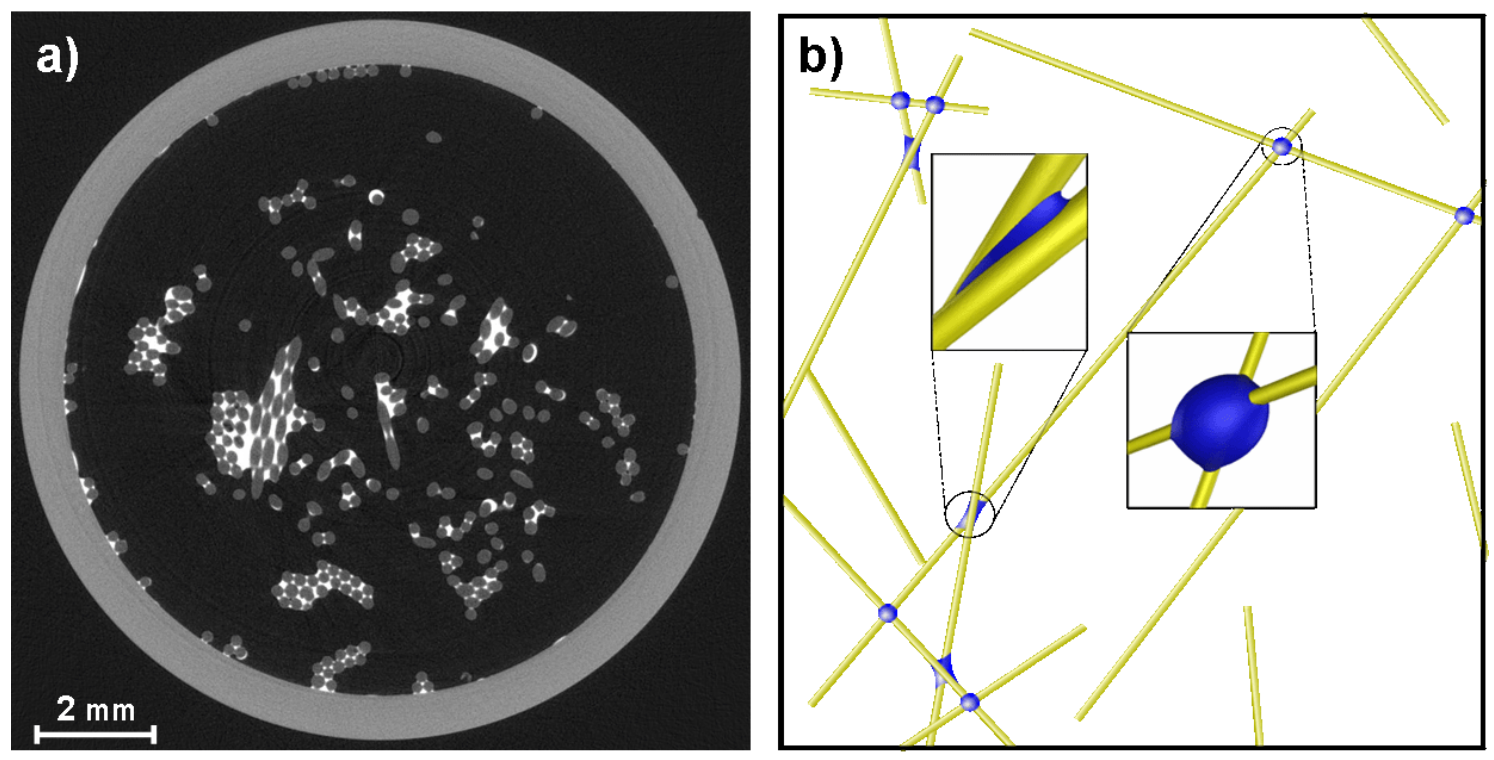

Figure 5.3: a) Slice though a X-ray micro tomography image of wet glass fibers. The fibers were $10 \mathrm{~mm}$ long and had a radius of $150 \mu \mathrm{m}$. b) Liquid morphologies between fibers obtained by numerical minimization of liquid clusters between fibers [60]. The insets show enlarged examples of capillary bridges between fibers. 


\subsubsection{Packing density of cylinders}

We analyzed X-ray micro tomography images and measured the packing density of cylinders at different aspect ratios. For this experiment, we produced cylinders with a diameter of $500 \mu \mathrm{m}$ and aspect ratios (length / diameter) in the range of 0.8 to 10 . X-ray micro tomography as shown in Figure 5.4 for the aspect ratios a) 1, b) 2, c) 6 .

Figure 5.4 d) shows the packing density of cylinders as a function of the aspect ratio. At an aspect ratio of 1 a clear maximum is visible at a packing density of $\rho=0.67$, which is larger then the RCP $\rho_{R C P}=0.64$ of beads. For comparison we show the the packing density of cylinders with the theory for ellipsoids investigated by Donev et al. [32]. They show calculated packing densities for various aspect ratios (length / diameter) from 0.3 to 3.5 i.e., from oblate to prolate. The theory of the packing density of ellipsoids shows a minimum at an aspect ratio of 1 , whereas the experimentally measured cylinder packing shows a maximum at this point. A theory for cylinder packing might be developed in future.

The X-ray micro tomography gives access to the number of contacts of ellipsoids or cylinders. The X-ray micro tomography is a useful technique for many fields where 3D information on a multiple phase system is needed. 


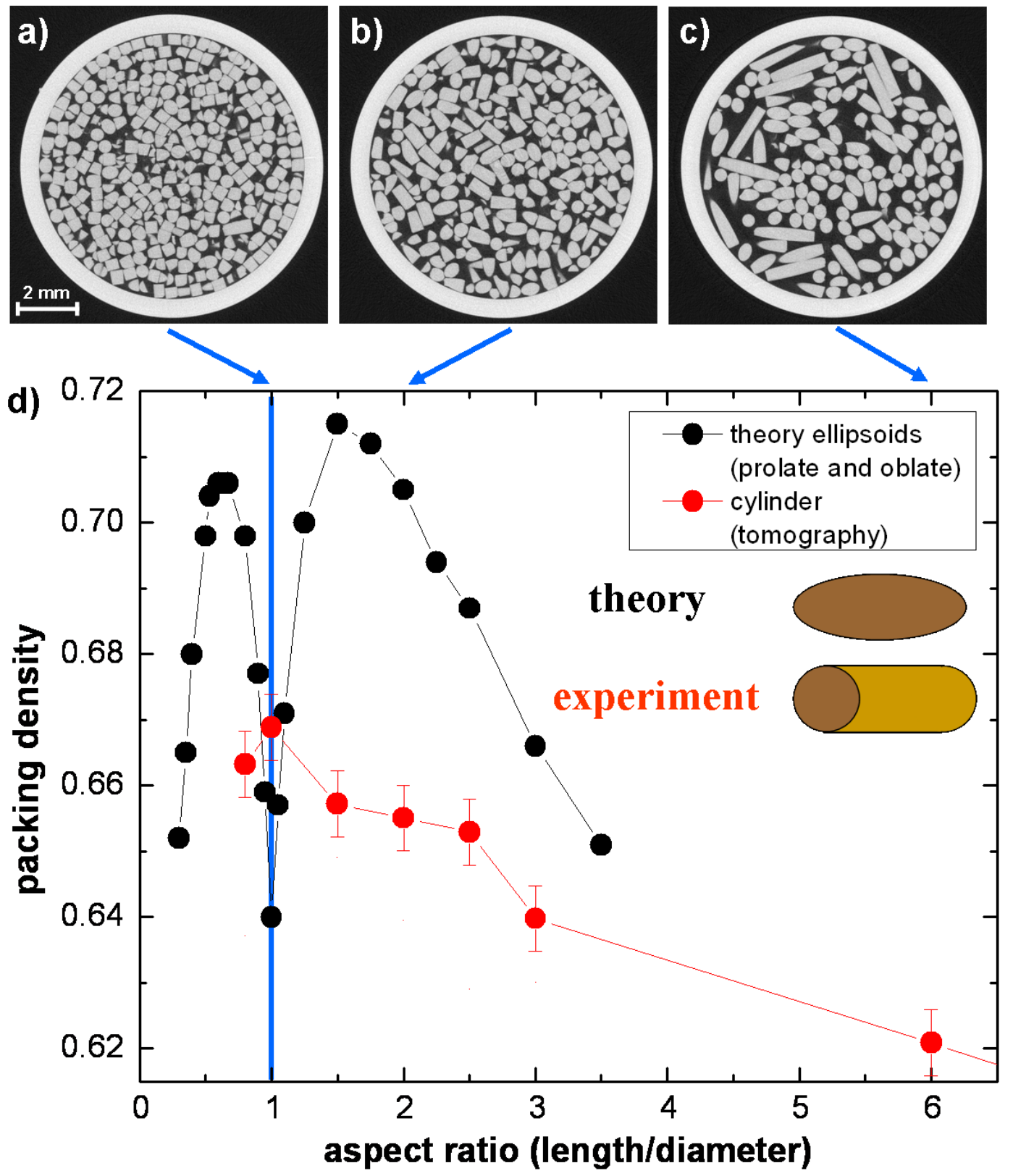

Figure 5.4: Section though three tomography images of glass cylinders with an aspect ratio of a) 1, b) 2 and c) 6. d) Packing density of cylinders as a function of the aspect ratio as obtained from the X-ray tomography images. For comparison we show the theoretically packing density of ellipsoids for various aspect ratios [32]. The cylinders show a maximum in the packing density of $\rho=0.67 \pm 0.01$ at aspect ratio of 1 , whereas the theory for ellipsoid packing shows a minimum at this density. 



\section{Appendices}





\section{Appendix A}

\section{Parameter of liquids and particles}

\section{Liquids}

\begin{tabular}{|c|c|c|c|c|}
\hline name & viscosity $[\mathrm{Pa} \cdot \mathrm{s}]$ & surface tension $[\mathrm{mN} / \mathrm{m}]$ & density $\left[\mathrm{g} / \mathrm{m}^{3}\right]$ & X-ray absorption \\
\hline \hline water & 1.0 & 72 & 1.0 & 0.2 \\
diiodomethane & 2.8 & 51 & 3.3 & 15 \\
dibromomehtane & 1.0 & 39 & 2.1 & 5 \\
ZnI $_{2}$ water & $1-40$ & $72-85$ & $1-2.6$ & $0.2-10$ \\
silicone oil & variable & 20 & 0.96 & 0.3 \\
olive oil & 84 & 36 & 0.9 & - \\
ethanol & 1.1 & 22 & 0.79 & 0.2 \\
hexan & 0.33 & 18 & 0.66 & - \\
nonan & 1.0 & 22 & 0.72 & - \\
ethylene glycol & 16 & 48 & 1.1 & - \\
toluene & 0.56 & 28 & 0.86 & - \\
decanol & 11 & 29 & 0.83 & - \\
\hline
\end{tabular}

Table A.1: Properties of used liquids.

Surface tension values were taken at $25^{\circ} \mathrm{C}$. Most of the parameters are from Handbook of Chemistry and Physics [88]. The properties of the salt solutions $\mathrm{ZnI}_{2}$ were measured. The $\mathrm{X}$-ray absorption in tomography images is normalized with the absorption of glass beads. This values depends strongly on the peak energy of the X-ray beam which was in our case $40 \mathrm{keV}$ at ID15A (ESRF). 


\section{Particles}

\begin{tabular}{|c|c|c|}
\hline name & density $\left[\mathrm{g} / \mathrm{m}^{3}\right]$ & X-ray absorption \\
\hline \hline polystyrene beads & 1.0 & 0.3 \\
PMMA beads & 1.2 & 0.4 \\
glass beads & 2.5 & 1.0 \\
basalt beads & 2.9 & 1.1 \\
ruby beads & 4.0 & 1.2 \\
steel & 7.9 & 10 \\
BaTi beads & 4.0 & 15 \\
gold beads & 19.3 & 200 \\
diamonds & 3.5 & 0.6 \\
quartz sand & 2.5 & 1 \\
NaCl cubes & 2.2 & 1.2 \\
\hline
\end{tabular}

Table A.2: Properties of used particles. 


\section{Bibliography}

[1] P. M. Vercruijsse. Developments in the dredging industry. Hydrotransport, 17:423, 2007.

[2] T. L. Youd at al. Liquefaction resistance of soils: Summary report from the 1996 NCEER and 1998 NCEER/NSF workshops on evaluation of liquefaction resistance of soils. J. Geotech. and Geoenviron. Eng., ASCE., 127:817, 2001.

[3] Y. Okura, H. Kitahara, H. Ochiai, T. Sammori, and A. Kawanami. Landslide fluidization process by flume experiments. Eng. Geology, 66:65, 2002.

[4] A. Khaldoun, E. Eiser, G. H. Wegdam, and D. Bonn. Rheology: Liquefaction of quicksand under stress. Nature, 437:635, 2005.

[5] D. Lohse, R. Rauhé, R.Bergmann, and D. v. d. Meer. Creating a dry variety of quicksand. Nature, 432:689, 2005.

[6] V. Schwämmle and H. J. Herrmann. A model of barchan dunes including lateral shear stress. Eur. Phys. J. E., 16:57, 2005.

[7] N. J. Middleton and A. S. Goudie. Saharan dust: Sources and trajectories. Trans. Inst. Br. Geogr. NS, 26:165, 2001.

[8] S. Douady, A. Manning, P. Hersen, H. Elbelrhiti, S. Protière, A. Daerr, and B. Kabbachi. Song of the dunes as a self-synchronized instrument. Phys. Rev. Lett., 97:018002, 2006.

[9] C. Liu, S. R. Nagel, D. A. Schecter, S. N. Coppersmith, S. Majumdar, O. Narayan, and T. A. Witten. Force fluctuations in bead packs. Science, 269:513, 1995.

[10] J. Geng, G. Reydellet, E. Clément, and R. P. Behringer. Green's function measurements of force transmission in 2D granular materials. Physica D, 182:274, 2003. 
[11] C. Goldenberg and I. Goldhirsch. Friction enhances elasticity in granular solids. Nature, 435:188, 2005.

[12] J. Geng, E. Longhi, R. P. Behringer, and D. Howell. Memory in two-dimenisional heap experiments. Phys. Ref. E, 64:60301, 2001.

[13] J. Brujic, S. F. Edwards, D. V. Grinev, I. Hopkinson, D. Brujic, and H. A. Makse. 3D bulk measurements of the force distribution in a compressed emulsion system. Faraday Discuss., 123:207, 2003.

[14] J. F. Peters, M. Muthuswamy, J. Wibowo, and A. Tordesillas. Characterization of force chains in granular material. Phys. Rev. E, 72:041307, 2005.

[15] J. Geng, D. Howell, R. P. Behringer, G. Reydellt, L. Vanel, E. Clément, and S. Luding. Footprints in sand: The response of a granular material to local perturbations. Phys. Ref. Lett., 87:035506, 2001.

[16] W. C. Krumbein and L. L. Sloss. Stratigraphy and Sedimentation, 2nd edition. Freeman, San Francisco, 1963.

[17] J. H. Conway and N. J. A. Sloane. Sphere Packings, Lattices, and Groups, 2nd ed. Springer-Verlag, New York, 1993.

[18] A. V. Anikeenko, N. N. Medvedev, A. Bezrukov, and D. Stoyan. Observation of fivefold symmetry structures in computer models of dense packing of hard spheres. J. of NonCry. Solids, 353:3545, 2007.

[19] G. D. Scott and D. M. Kilgour. The density of random close packing of spheres. J. Phys. D, 2:863, 1969.

[20] J. G. Berryman. Random close packing of hard spheres and disks. Phys. Rev. A, 27:1053, 1983.

[21] H. M. Jaeger and S. R. Nagel. Physics of granular states. Science, 255:1524, 1992.

[22] J. B. Knight, C. G. Fandrich, C. N. Lau, H. M. Jaeger, and S. R. Nagel. Density relaxation in a vibrated granular material. Phys. Ref. E, 51:3957, 1995.

[23] J. Q. Xu, R. P. Zou, and A. B. Yu. Packing structures of cohesive spheres. Phys. Ref. E, 69:032301, 2004. 
[24] P. Richard, M. Nicodemi, R. Delannay, P. Ribiére, and D. Bideau. Slow relaxation and compation of granular systrems. Nature Materials, 4:121, 2005.

[25] K. Chen, J. Cole, C. Conger, J. Draskovic, M. Lohr, K. Klein, T. Scheidemantel, and P. Schiffer. Packing grains by thermally cycling. Nature, 442:257, 2006.

[26] G. Y. Onoda and E. G. Lininger. Random loose packings of uniform spheres and the dilatancy onset. Phys. Rev. Lett., 64:2727, 1990.

[27] A. P. Shapiro adn R. F. Probstein. Random packings of spheres and fluidity limits of monodisperse and bidisperse suspensions. Phys. Rev. Lett., 68:1422, 1992.

[28] S. Andersson and M. O'Keeffe. Body-centred cubic cylinder packing and the garnet structure. Nature, 267:605, 1977.

[29] J. Blouwolff and S. Fraden. The coordination number of granular cyliders. Europhys. Lett., 76:1095, 2006.

[30] F. X. Villarruel, B. E. Lauderdale, D. M. Mueth, and H. M. Jaeger. Compaction of rods: Relaxation and ordering in vibrated, anisotropic granular materials. Phys. Ref. E, 61:6914, 2000.

[31] R. P. Zou and A. B. Yu. Evaluation of the packing characteristic of mono-sized nonspherical particles. Pow. Tech., 88:71, 1996.

[32] A. Donev, I. Cisse, D. Sachs, E. A. Variano, F. H. Stillinger, R. Connelly, S. Torquato, and P. M. Chaikin. Improving the density of jammed disordered packings using ellipsoids. Science, 303:990, 2004.

[33] J. N. Israelachvili. Intermolecular Surface Forces. Acedemic Press, New York, 1992.

[34] D. Willett, M. J. Adams, S. A. Johnson, and J. P. K. Seville. Capillary bridge between two sperical bodies. Langmuir, 16:9396, 2000.

[35] J. Lee and C. J. Kim. Surface - tension - driven microactuation based on continous electrowetting. J. Microelectromech. Syst., 9:171, 2000.

[36] T. A. Mcmahon and J. T. Bonner. On Size and Life. Scientific American Books, New York, 1983. 
[37] W. S. N. Trimmer. Microrobots and micromechanical systems. Sensor and Actuators, 19:267, 1989.

[38] P. G. Gennes. Rev. Mod. Phys., 57:827, 1985.

[39] T. Young. Phil. Tran. Royal Soc., part I:65, 1805.

[40] S. Safran. Statistical Thermodynamics of Surfaces. Interfaces And Membranes, Addison-Wesley Publishing Company, New York, 1994.

[41] M. Schick. Liquids at Interfaces. Elsevier Science, Amsterdam, 1889.

[42] S. Dietrich. Phase Transition and Critical Phenomena, Vol. 12. Academic Press, London, 1988.

[43] A. Dujbré. Theory Mechanique de la Chaleur. Gauthier-Villars, Paris, 1869.

[44] G. Mason and W. C. Clark. Liquid bridges between spheres. Chem. Eng. Sci., 20:859, 1965.

[45] F. M. Orr, L. E. Scriven, and A. P. Rivas. Pendular rings between solids: meniscus properties and capillary force. J. Fluid Mech., 67:723, 1975.

[46] W. B. Haines. Studies of the physical properties of soils. II. a note on the cohesion developed by capillary forces in an ideal soil. J. of Agric. Sci., 15:529, 1925.

[47] G. Lian, C. Thornton, and M. J. Adams. A theoretical study of the liquid bridge forces between two rigid spherical bodies. J. Coll. Int. Sci., 161:138, 1993.

[48] N. Garoff and S. Zauscher. The influence of fatty acids and humidity on friction and adhesion of hydrophilic polymer surfaces. Langmuir, 18:6921, 2002.

[49] H. Schubert. Kapillarität in porösen Feststoffsystemen. Springer - Verlag, Berlin Heidelberg New York, 1982.

[50] N. L. Cross and R. G. Picknett. Particle adhesion in the presence of a liquid film. In the Mechanism of Corrosion by Fuel Impurities, H. R. Johnson, D. H. Littler. London. Butterworths, 1963.

[51] T. C. Halsey and A. J. Levine. How sandcastles fall. Phys. Rev. Lett., 80:3141, 1998. 
[52] B. L. Weeks, M. W. Vaughn, and J. J. DeYoreo. Direct imaging of meniscus formation in atomic force microscopy using environmental scanning electron microscopy. Langmuir, 21:8096, 2005.

[53] M. Schenk, M. Füting, and R. Reichelt. Direct visualization of the dynamic behavior of a water meniscus by scanning electron microscopy. J. of App. Phys., 84:4880, 1998.

[54] M. Schwamberger. Wetting morphologies in random assemblies of spheres. Diploma thesis, 2008.

[55] C. L. Feng and A. B. Yu. Quantification of the relationship between porosity and interparticle force for the packing of wet uniform spheres. J. Col. and Int. Sci., 231:136, 2000.

[56] C. C. Harris and N. R. Morrow. Pendular moisture in packings of equal spheres. Nature, 203:706, 1964.

[57] R. A. Fisher. On the capillary forces in an ideal soil; correction of formulae given by W. B. Haines. J. Agric. Sci., 16:492, 1926.

[58] S. Herminghaus. Dynamics of wet granlar mattter. Adv. Phys., 54:221, 2005.

[59] N. Mitarai and F. Nori. Wet granular materials. Adv. Phys., 55:1, 2006.

[60] M. Brinkmann. Paper in preparation, 2009.

[61] K. Brakke. The Surface Evolver and the stability of liquid surfaces. Phil. Trans. R. Soc. A, 354:2143, 1996.

[62] M. Scheel, R. Seemann, M. Brinkmann, M. Di Michiel, A. Sheppard, B. Breidenbach, and S. Herminghaus. Morphological clues to wet granular pile stability. Nature Materials, 7:189, 2008.

[63] M. Di Michiel, J. M. Merino, D. Fernandez-Carreiras, T. Buslaps, V. Honkimäki, and P. Falus. Fast microtomograhy using high energy synchrotron radiation. Rev. Sci. Instrum., 76:043702, 2005.

[64] A. Frangakis and R. Hegerl. Noise reduction in electron tomographic reconstructions using nonlinear anisotropic diffusion. J. Struct. Biol., 135:239, 2001. 
[65] A. Sheppard, R. M. Sok, and H. Averdunk. Techniques for image enhancement and segmentation of tomographic images of porous materials. Physica A, 339:145, 2004.

[66] J. A. Sethian. Level Set Methods and Fast Marching Methods: Evolving Interfaces in Computational Geometry, Fluid Mechanics, Computer Vision and Materials Science. Cambridge University Press, Cambridge, 1999.

[67] P. Schiffer. A bridge to sandpile stability. Nature, 1:21, 2005.

[68] S. Nowak, A. Samadani, and A. Kudrolli. Maximum angle of stability of a wet granular pile. Nature Physics, 1:50, 2005.

[69] P. Tegzes, T. Vicsek, and P. Schiffer. Avalanche dynamics in wet granular material. Phys. Rev. Lett., 89:094301, 2002.

[70] L. Bocquet, E. Charlaix, and F. Restagno. Physics of humid granular media. C. R. Physique, 3:207, 2002.

[71] L. Bocquet, E. Charlaix, S. Ciliberto, and J. Crassous. Moisture-induced ageing in granular media and the kinetics of capillary condensation. Nature, 396:735, 1998.

[72] F. Cherblanc F. Soulié, M. S. El Youssoufi and C. Saix. Capillary cohesion and mechanical strength of polydisperse granular materials. Eur. Phys. J. E, 21:349, 2007.

[73] P. Pierrat and H. S. Caram. Tensile strength of wet granular materials. Powders Technology, 91:83, 1997.

[74] V. Richefeu, M. S. El Youssoufi, and F. Radjaï. Shear strength properties of wet granular materials. Phys. Rev. E, 73:051304, 2006.

[75] N. Lu, B. Wu, and C. P. Tan. Tensile strength characteristics of unsaturated sands. J. Geotech. and Geoenvir. Eng., 133:144, 2007.

[76] D. Geromichalos. Dynamik feuchter Granulate. PhD thesis. Univeristy Ulm, 2004.

[77] G. H. Ristow. Pattern Formation in Granular Material. Springer, Berlin, 2000.

[78] J. Duran. Sands, Powders and Grains. Spriner, New York, USA, 2000. 
[79] M. Scheel, R. Seemann, M. Brinkmann, M. Di Michiel, A. Sheppard, and S. Herminghaus. Liquid distribution and cohesion in wet granular assemblies beyond the capillary bridge regime. J. Phys.: Cond. Matter, 20:494246, 2008.

[80] D. J. Hornbaker, R. Albert, I. Albert, A.-L. Barabási, and P. Schiffer. What keeps sandcastles standing? Nature, 387:765, 1997.

[81] Z. Fournier, D. Geromichalos, S. Herminghaus, M. M. Kohonen, F. Mugele, M. Scheel, M. Schulz, B. Schulz, Ch. Schier, R. Seemann, and A. Skudelny. Mechanical properties of wet granular matgerials. J. Phys.: Cond. Matter, 17:477, 2005.

[82] J. S. McFarlane and D. Tabor. Adhesion of solids and effect of surface films. Proceedings of the Royal Society of London Series a-Mathematical and Physical Sciences., 202:224, 1950.

[83] N. L. Cross and R. G. Picknett. The liquid layer between a sphere and a plane surface. Trans. Faraday Soc., 59:846, 1963.

[84] W. C. Clark, J. M. Haynes, and G. Mason. Liquid bridges between a sphere and a plane. Chem. Eng. Sci., 23:810, 1968.

[85] B. Derjaguin. Analysis of friction and adhesion. iv. the theory of adhesion of small particles. Kolloid-Zeitschrift., 69:155, 1934.

[86] O. Pitois, P. Moucheront, and X. Chateau. Liquid bridge between two moving spheres: An experimental study of viscosity effects. J. of Col. Int. Sci., 231:26, 2000.

[87] O' Brian and W. J. Hermann. Stength of liquid bridges between dissimilar materials. Journal of Adhesion, 10:91, 1973.

[88] D. R. Lide. Handbook of Chemistry and Physics, 86th edition 2005 - 2006. Taylor and Francis, New York, 2005.

[89] P. C. F. Møller and D. Bonn. The shear modulus of wet granular matter. EPL, 80:38002, 2007.

[90] P. C. F. Møller, J. Mewis, and D. Bonn. Yield stress and thixotropy: on the difficulty of measuring yield stresses in practice. Soft Matter, 2:274, 2006. 
[91] M. Sipahi. Messungen an feuchten Granulaten mit einer Scherzelle. Examination, 2009.

[92] T. Aste, M. Saadatfar, and T. J. Senden. Local and global relations between the number of contacts and density in monodisperse sphere packs. J. Stat. Mech., :P07010, 2006.

[93] H. Rumpf. The strength of granules and agglomerates. Agglomeration, Edited by W. A. Knepper (Interscience, New York), page 379, 1962.

[94] M. Scheel, D. Geromichalos, and S. Herminghaus. Wet granular matter under vertical agitation. J. Phys.: Cond. Matter, 16:4213, 2004.

[95] C. F. Moukarzel. Isostatic phase transition and instability in stiff granular materials. Phys. Rev. Lett., 81:1634, 1998.

[96] G. Mason. Radial distribution function from small packings of spheres. Nature, 217:733, 1968.

[97] G. Mason and W. C. Clark. Distribution of near neighbours in a random packing of spheres. Nature, 207:512, 1965.

[98] J. D. Bernal and J. Mason. Co-ordination of randomly packed spheres. Nature, 185:68, 1960.

[99] T. Gröger, U. Tüzün, and D. M. Heyes. Modelling and measuring of cohesion in wet granular materials. Powders Technol., 133:203, 2002.

[100] M. M. Kohonen, D. Geromichalos, M. Scheel, Ch. Schier, and S. Herminghaus. On capillary bridges in wet granular materials. Physica A, 339:7, 2004.

[101] M. M. Kohonen, N. Maeda, and H. K. Christenson. Kinetics of capillary condensation in a nanoscale pore. Phys. Rev. Lett., 82:4667, 1999.

[102] P. Tegzes, R. Albert, M. Paskvan, A.-L. Barabási, T. Vicsek, and P. Schiffer. Liquidinduced transitions in granular media. Phys. Rev. E, 60:5823, 1999. 


\section{Acknowledgments}

The successful completion of this thesis would not have been possible without the contribution and help from lots of different people. First of all, I would like to thank my supervisor Prof. Stephan Herminghaus for giving me the opportunity to conduct my research under his supervision.

Thank to my second Supervisor Prof. Marcus Müller for guiding me in all cases.

I fall short of words while thanking my Guide Prof. Ralf Seemann, for helping me along the whole journey of my PhD. He gave me all the freedom and independence to work.

My life would be never so easy without the help and collaboration of Dr. Martin Brinkmann. Even in busy times, he always helped me with his strong theoretical and computational skills. Without his theoretical support my thesis would never have been a complete thesis.

Special thank goes to Dr. Marco Di Michiel for all of his work at the beamline ID15A and to Dr. Zeina Khan who worked with me on fluidization measurements and read my thesis carefully. Also to Sonia May and Markus Feder for reading my thesis carefully.

I would also thank the people who worked on granulates, Marcus Schwamberger, Dr. Matthias Schröter, Dr. Adrian Sheppard, Michael Sipahi, Dr. Boris Breidenbach, Dr. Vasily Zaburdaev. I thank all my roommates Venkat, Enkhtuul, Klaus Röller, Dr. Evgeny Gurevich, Dr. Axel Hager-Fingerle, and Anika Döring. I would also thank especially to Dr. Krishnacharya, Dr. Jürgen Vollmer, Dr. Audrey Steineberger, Dr. Dagmar Steinhauser and all from MPI-DS for their priceless support during my stay in Göttingen. I wish them all a good luck for their future.

It would be incomplete without thanking my parents and family who did every possible thing to make me reach at this stage of my life.

Mario. 



\section{Scientific Papers Published :}

1. M. Scheel, R. Seemann, M. Brinkmann, M. DI Michiel, A. Sheppard, S. Herminghaus, 'Liquid distribution and cohesion in wet granular assemblies beyond the capillary bridge regime', J. Phys.: Cond. Matter 20, 494246 (2008).

2. M. Scheel, R. Seemann, M. Brinkmann, M. DI Michiel, A. Sheppard, B. Breidenbach, S. Herminghaus, 'Morphological clues to wet granular pile stability', Nature Materials 7, 189, (2008).

3. Z. Fournier, D. Geromichalos, S. Herminghaus, M. M. Kohonon, F. Mugele, M. Scheel, M. Schulz, B. Schulz, Ch. Schier, R. Seemann, A. Skudelny, 'Mechanical properties of wet granular materials', J. Phys.: Cond. Matter 17, 477, (2005).

4. M. M. Kohonen, D. Geromichalos, M. Scheel, C. Schier, S. Herminghaus, 'On capillary bridges in wet granular materials', Physica A 339, 7, (2004).

5. M. Scheel, D. Geromichalos and S. Herminghaus, 'Wet granular matter under vertical agitation', J. Phys.: Cond. Matter 16, 4213, (2004). 



\section{Lebenslauf}

\section{Persönlicher Status}

Name : $\quad$ Mario Scheel

Geburtsdatum : $\quad$ 04. Januar 1977

Geburtsort : $\quad$ Kempten (Allgäu), Deutschland

Wohnort : $\quad$ Hinter den Höfen 31, 37124 Rosdorf, Deutschland

Staatsangehörigkeit : Deutsch

\section{Ausbildung}

$2005-2009$

Doktorarbeit am Max-Planck Institut für Dynamik und Selbstorganisation, Göttingen. 'Experimental investigations of the mechanical properties of wet granular matter'

Referent: Prof. Dr. Stephan Herminghaus

Koreferent: Prof. Dr. Marcus Müller

Betreuer: Prof. Dr. Ralf Seemann

2004 - 2005 Diplomarbeit am Max-Planck Institut für Dynamik und Selbstorganisation, Göttingen. 'Struktur und Dynamik feuchter Granulate' Betreuer: Dr. Ralf Seemann und Prof. Dr. Stephan Herminghaus

1999 - $2004 \quad$ Physik Studium an der Universität Ulm.

1998 - 1999 Zivildienst, Tom Mutters Schule, Kempten (Allgäu).

1996 - 1998 Schulbildung, Staatl. Berufsoberschule Kempten (Allgäu).

1993 - 1996 Beruf, Energieelektroniker Fachrichtung Betriebstechnik.

1989 - 1993 Schulbildung, Staatl. Realschule für Knaben Kempten (Allgäu). 